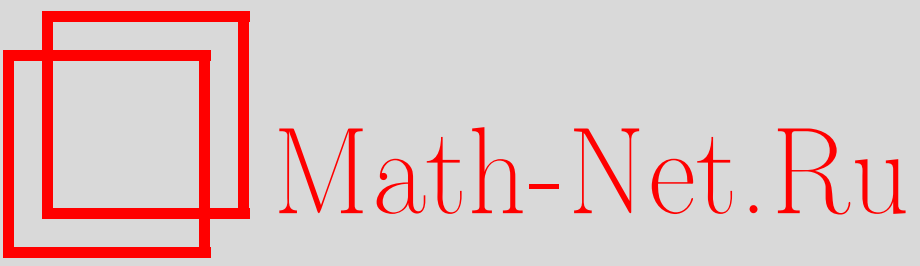

А. Г. Хованский, О разрешимости и неразрешимости уравнений в явном виде, УМН, 2004, том 59, выпуск 4, 69-146

DOI: https://doi.org/10.4213/rm759

Использование Общероссийского математического портала Math-Net.Ru подразумевает, что вы прочитали и согласны с пользовательским соглашением

http://www . mathnet.ru/rus/agreement

Параметры загрузки:

IP: 35.174 .16 .151

26 апреля 2023 г., 04:06:51 


\section{О РАЗРЕШИМОСТИ И НЕРАЗРЕШИМОСТИ УРАВНЕНИЙ В ЯВНОМ ВИДЕ}

\section{А. Г. ХОВАНСКИЙ}

В обзоре обсуждаются классические результаты Абеля, Лиувилля, Галуа, Пика$\mathrm{pa}$, Вессио, Колчина и др. о разрешимости и неразрешимости уравнений в явном виде. Подробно излагается одномерный топологический вариант теории Галуа, описывающий топологические препятствия для представимости функций в квадратурах.

Библиография: 37 названий.

\section{СОДЕРЖАНИЕ}

$\S 1$. Постановка задачи о разрешимости уравнений в конечном виде ......

1.1. Задание класса функций с помощью списков основных функций и допустимых операций

1.2. Классические классы функций одной переменной _............... 74

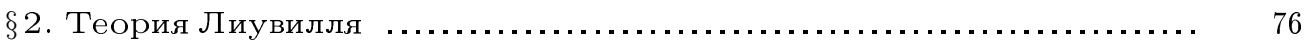

2.1. Новые определения классических классов функций $\ldots \ldots \ldots \ldots \ldots \ldots . . . . . .6$

2.2. Расширения Лиувилля абстрактных и функционалшных дифференциальных полей . . . . . . . . . . . . . . . . . . . . . . . . . . . . . . . . . . .

2.3. Некоторые резултаты теории Лиувилля

$\S 3$. Разрешимость алгебраических уравнений в радикалах и теория Га-

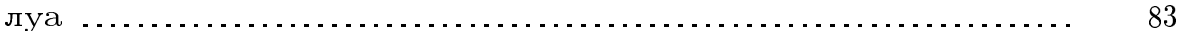

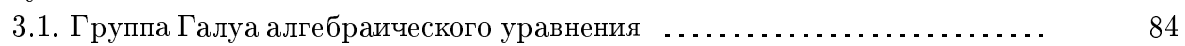

3.2. Основная теорема теории Галуа ................................ 85

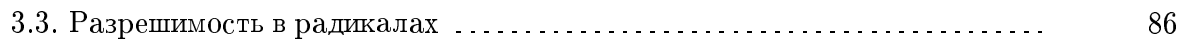

3.4. Понижение степени уравнения ................................. 90

$\S 4$. Разрешимость линейных дифференциальных уравнений в квадратурах и теория Пикара-Вессио

4.1. Аналогия между линейными дифференциальными уравнениями и алгеб-

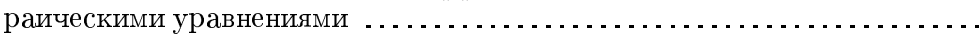

4.2. Группа Галуа линейного дифференциального уравнения . . . . . . . . . . . .

4.3. Основная теорема теории Пикара-Вессио ....................... 98

4.4. Простейшие расширения Пикара-Вессио .......................... 100

4.5. Разрешимость дифференциальных уравнений ...................... 102

4.6. Алгебраические матричные группы и необходимые условия разрешимос-

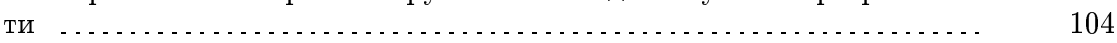

4.7. Достаточное условие разрешимости дифференциальных уравнений .... 105

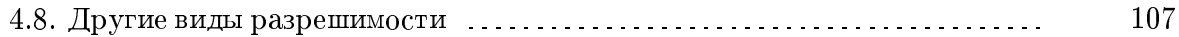


$\S 5$. Одномерный топологический вариант теории Галуа $\ldots \ldots \ldots \ldots \ldots \ldots \ldots . .110$

5.1. Предварительные замечания .............................. 110

5.2. Функции с не более чем счетным множеством особых точек . . . . . . . . . . 117

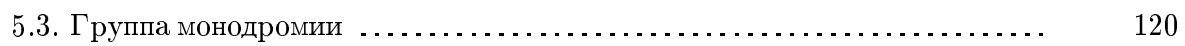

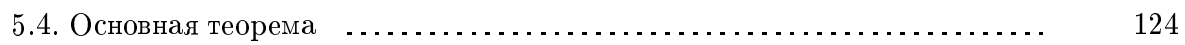

5.5. Групповые препятствия к представимости в квадратурах $\quad . . . . . . . . . \quad 126$

§6. Разрешимость в квадратурах линейных дифференциальных уравнений типа Фукса и топологический вариант теории Галуа ........ 132

6.1. Теория Пикара-Вессио для уравнений типа Фукса ... . . . . . . . . . . . . 132

6.2. Теория Галуа систем линейных дифференциальных уравнений типа Фукса

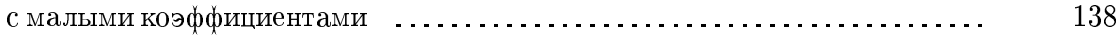

Список литературы . . . . . . . . . . 145

Многочисленные неудачные попытки решения ряда алгебраических и дифференциальных уравнений “в явном виде" привели математиков к убеждению, что явных решений для этих уравнений просто не сушествует. Настояший обзор посвящен вопросу о неразрешимости уравнений в явном виде. Этот вопрос имеет богатую историю.

Первые доказательства неразрешимости алгебраических уравнений в радикалах были найдены Абелем и Галуа. Обдумывая задачу о нахождении в явном виде неопределенного интеграла от алгебраической дифференциальной формы, Абель заложил основы теории алгебраических кривых. Лиувилль продолжил работы Абеля и доказал неэлементарность неопределенных интегралов многих алгебраических и элементарных дифференциальных форм. Неразрешимость в квадратурах ряда линейных дифференциальных уравнений тоже впервые была доказана Лиувиллем.

Еше Галуа связал вопрос о разрешимости в радикалах со свойствами некоторой конечной групшы (так называемой групшы Галуа алгебраического уравнения). Собственно, само понятие конечной группы было введено Галуа именно в связи с этим вопросом. Софус Ли ввел понятие непрерывной группы преобразований, пытаясь явно решать дифференциальные уравнения и приводить их к более простому виду. Пикар с каждым линейным дифференциальньм уравнением связал его группу Галуа, которая является групой Ли (и, более того, является алгебраической матричной группй). Пикар и Вессио показали, что именно эта группа отвечает за разрешимость уравнений в квадратурах. Колчин развил теорию алгебраических групп и придал теории Пикара-Вессио законченньй вид.

Арнольд обнаружил, что ряд классических вопросов математики неразрешимы из-за топологических причин. В частности, он показал, что общее алгебраическое уравнение степени $\geqslant 5$ не решается в радикалах именно по топологическим причинам. Я бесконечно признателен Владимиру Игоревичу за то, что он заинтересовал меня этой тематикой. Развивая подход Арнольда, в начале семидесятых годов я построил своеобразный одномерньй топологический вариант теории Галуа. Согласно этой теории топология расположения римановой поверхности аналитической функции над плоскостью комплексного переменного может препятствовать представимости этой функции при помоши явных формул. На этом пути получаются наиболее сильные из известных результатов о непредставимости функщий явными формулами. Недавно мне удалось обобщить эти топологические результаты на случай многих переменных.

\footnotetext{
Работа выполнена при частичной поддержке гранта OGP 0156833 (Канада).
} 
В обзоре дается полное изложение одномерного топологического варианта теории Галуа. Этот вариант тесно связан как с обычной теорией Галуа, так и с теорией Пикара-Вессио. Конечно, полностью изложить эти классические теории в статье невозможно. Основные теоремы этих теорий формулируются без доказательств, зато подробно объясняется, почему эти теории в принщипе отвечают на вопросы о разрешимости алгебрических уравнений в радикалах и о разрешимости линейных дифференциальных уравнений в квадратурах. В обзоре обсуждается также красивое построение Лиувилля класса элементарных функций, класса функций, представимых в квадратурах, и т. д. и его теория, оказавшая большое влияние на все дальнейшие работы в этой области.

Итак, в работе идет речь о трех вариантах теории Галуа - обычном, дифференщиальном и топологическом. Эти варианты объединяет общий подход к задачам о разрешимости и неразрешимости уравнений, основанный на теории групп. Неверно, однако, что все результаты о разрешимости и неразрешимости связаны с теорией групп. Ряд ярких результатов, основанных на другом подходе, содержится в теории Лиувилля.

В обзоре не всегда соблюдается историческая последовательность. Например, теорема Пикара-Вессио о разрешимости линейных дифференциальных уравнений в квадратурах была доказана раньше, чем основная теорема дифференциальной теории Галуа. Однако теорема Пикара-Вессио является прямым следствием этой основной теоремы, и именно так она представляется в настояшем обзоре.

Несколько слов о литературе. Изложение метода Лиувилля и близких работ Чебьшёва, Мордухай-Болтовского и др. можно найти в замечательной книге [33]. Обычная теория Галуа хорошо излагается во многих местах. Короткое и ясное изложение дифференциальной теории Галуа содержится в [17]. Интересньй обзор работ о разрешимости и неразрешимости уравнений вместе с обширной библиографией можно найти в [36].

В свое время я не опубликовал полное изложение одномерного топологического варианта теории Галуа: сначала я не мог разобраться в сложной истории предмета, а потом занялся совсем другой математикой. Много лет спустя Андрей Болибрух попросил меня вернуться к этой теме и подготовить работу для публикации в своем новом журнале. Часть работы была подготовлена тогда же [22]. Здесь работа излагается полностью, обсуждаются ее взаимосвязи с классическими вариантами теории Галуа, приводится новая постановка задачи, необходимая для построения многомерного варианта теории. Без вмешательства Болибруха, скорее всего, одномерньй вариант так и не был бы подготовлен к публикации, а многомерньй вариант не был бы найден. Я признателен моей жене Т. В. Белокринищкой за помощь при подготовке этого обзора.

Этот обзор посвящается памяти Андрея Андреевича Болибруха - замечательного человека и первоклассного математика.

\section{$\S$ 1. Постановка задачи о разрешимости уравнений в конечном виде}

Некоторые алгебраические и дифференциальные уравнения "решаются явно". Что это значит? Если решение предъявлено, оно само и дает ответ на этот вопрос. Обычно все же попытки явного решения уравнений оказьваются безуспешными. Возникает желание доказать, что для тех или иных уравнений явных решений не сушествует. Тут 
уже просто необходимо точно определить, о чем идет речь (иначе непонятно, что, собственно, мы собираемся доказать). С современной точки зрения в классических работах недостает четких определений и формулировок теорем. Лиувилль, несомненно, точно понимал, что он доказьвает. Он не только сформулировал задачи о разрешимости уравнений в элементарных функциях и квадратурах, но и алгебраизировал эти задачи. После его работ все эти понятия удалось определить над любым дифференциальным полем. Но требования к математической строгости во времена Лиувилля были не такие, как сейчас. Согласно Колчину (см. [26]), даже у Пикара основные определения еще недостаточно продуманы. Работы Колчина вполне современны, но его определения с самого начала даются для абстрактных дифференциальных полей.

Все же решения дифференциального уравнения - это функции, а не элементы абстрактного дифференциального поля. В функциональных пространствах, кроме дифференцирования и арифметических операций, есть, например, абсолютно неалгебраическая операция суперпозиции. Вообше, в функциональных пространствах больше средств для написания "явных формул", чем в абстрактных дифференщиальных полях. Кроме этого, приходится учитьвать, что функции бьвают многозначньми, имеют особенности и т. д.

Формализовать задачу о неразрешимости уравнений в явном виде в функциональных пространствах несложно (в обзоре мы будем интересоваться именно этой задачей). Сделать это можно так: можно выделить тот или иной класс функций и сказать, что уравнение решается явно, если его решение принадлежит этому классу. Разные классы функций соответствуют разным понятиям разрешимости.

1.1. Задание класса функций с помощью списков основных функций и допустимых операций. Класс функций можно выделить, задав список основных функций и список допустимых операций. После этого класс функций определяется как множество всех функций, которые получаются из основных функций при помоши применения допустимых операций. В п. 1.2 именно таким способом определяются классические классы функций.

Классические классы функций, фигурируюшие в задачах о разрешимости в конечном виде, содержат многозначные функции. В связи с этим исходные понятия нужно уточнить. В этом пункте приведены два варианта такого уточнения. При первом чтении этот пункт можно пропустить.

Итак, пусть фиксирован класс основных функций и запас допустимых операций. Выражается ли заданная функция (яляюшаяся, скажем, решением данного алгебраического или дифференщиального уравнения или возникшая из каких-либо других соображений) через основные функци с помошью допустимых операций? Нас интересуют различные однозначные ветви многозначных функций над различньми областями. Каждую функцию, даже если она является многозначной, мы будем рассматривать как совокупность всех ее однозначных ветвей. Мы будем применять допустимые операции (такие как арифметические операции или операция взятия суперпозиций) лишь к однозначным ветвям функций над различньми областями. Так как мы имеем дело с аналитическими функциями, то в качестве областей достаточно рассматривать лишь малые окрестности точек.

Вопрос теперь видоизменяется следуюшим образом: выражсается ли заданныи росток функции в заданной точке через ростки основных функций при помощи 
доnустимых операций? Конечно, ответ зависит от выбора точки и от выбора однозначного ростка в этой точке заданной многозначной функции. Однако оказьвается, что (для интересуюших нас классов функций) либо искомого выражения не существует ни для какого ростка заданной многозначной функции ни в какой точке, либо, наоборот, “одно и то же” представление обслуживает все ростки заданной многозначной функции почти в любой точке пространства. В первом случае мы будем говорить, что никакая ветвь заданной многозначной функции не выражсается через ветви основных функиий при помощи допустимых операций. Во втором случае мы будем говорить, что такое выражение существует.

Возможен и другой, “глобальный” вариант работы с многозначными функциями, приводящий к другому (несколько расширенному) пониманию определения класса функций, заданного списками основных функций допустимых операций. В этом глобальном варианте многозначная функция рассматривается как единый объект. Определяются операции над многозначными функциями. Результат применения этих операций тоже многозначен: результатом является некоторое множество многозначных функций, про каждую из которых говорится, что она получена применением заданной операции к заданным функциям. Класс функций определяется как множество всех (многозначных) функций, которые получаются из основных функций при помоши допустимых операций.

Определим, например, что такое сумма двух многозначных функций одной переменной в смысле этого варианта.

ОПРЕДЕЛЕНИЕ. Возьмем произвольную точку $а$ на комплексной прямой, один из ростков $f_{a}$ аналитической функции $f$ в точке $a$ и один из ростков $g_{a}$ аналитической функции $g$ в той же точке $a$. Будем говорить, что многозначная функция $\varphi$, порожденная ростком $\varphi_{a}=f_{a}+g_{a}$, представима в виде суммы функций $f$ и

Например, легко видеть, что ровно две функции представляются в виде $\sqrt{x}+\sqrt{x}$, это $f_{1}=2 \sqrt{x}$ и $f_{2} \equiv 0$. Абсолютно аналогично определяются и другие операции над многозначными функциями. Замкнутость какого-либо класса многозначных функций относительно сложения означает, что этот класс вместе с любыми двумя функииями содержит все функиии, представимые в виде их суммы. То же самое нужно сказать и про все другие операции над многозначными функциями, понимаемые в указанном выше смысле.

В приведенном вьше определении важную роль играет не только сама операция сложения, но и операция аналитического продолжения, спрятанная в понятии многозначной функщии. Действительно, рассмотрим следующий пример. Пусть $f_{1}-$ аналитическая функция, определенная в области $U$ комплексной прямой $\mathbb{C}^{1}$, не продолжаюшаяся аналитически за пределы области $U$, и пусть $f_{2}$ - аналитическая функция в области $U$, определенная равенством $f_{2}=-f_{1}$. Согласно данному определению, функция, тождественно равная нулю, представима в виде $f_{1}+f_{2}$ на всей комплексной прямой. Согласно общепринятой точке зрения, равенство $f_{1}+f_{2}=0$ справедливо только в области $U$, но не вне ее.

В глобальном варианте работы с многозначньми функциями мы не настаиваем на сушествовании единой области, в которой все нужные действия производились бы над однозначными ветвями многозначных функций. Одна операция может производиться в одной области, а другая операция - в другой области над аналитическими 
продолжениями полученных функций. В сушности, это расширенное понимание операций эквивалентно добавлению операции аналитического продолжения к числу допустимых операций над аналитическими ростками. Для функции одной переменной удается получить топологические ограничения даже и при таком, расширенном, понимании операций над многозначными аналитическими функциями. Говорить о функциях чуть короче, чем говорить о ростках (так как не надо фиксировать точку, в которой рассматривается росток, и не надо уточнять, о каком именно ростке многозначной функции идет речь). Поэтому ниже при рассмотрении топологических препятствий к принадлежсности функции одной переменной тому или иному классу мы будем иметь в виду глобальный вариант определения класса функций с помощью списков основньх функиий и допустимых операчий. Для функций многих переменных в столь расширенной формулировке это сделать не удается и приходится принять более ограничительную формулировку, связанную с ростками функций, которая, впрочем, не менее (а может быть, даже более) естественна.

1.2. Классические классы функций одной переменной. Перечислим классические классы функций одной переменной. Мы будем задавать эти классы при помощи списков основных функций и допустимых операций.

Функции одной переменной, представимые в радикалах.

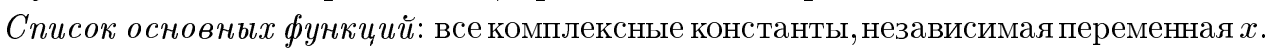

Cnисож допустимых операций: арифметические операции и операции извлечения корня $\sqrt[n]{f}$ степени $n, n=2,3, \ldots$, из заданной функции $f$.

Функция $g(x)=\sqrt[3]{5 x+2 \sqrt[2]{x}}+\sqrt[7]{x^{3}+3}$ доставляет пример функции, представимой в радикалах.

С этим классом связана знаменитая задача о разрешимости уравнений в радикалах. Рассмотрим алгебраическое уравнение

$$
y^{n}+r_{1} y^{n-1}+\cdots+r_{n}=0
$$

в котором $r_{i}$ - рациональные функции одной переменной. Полньй ответ на вопрос о разрешимости таких уравнений в радикалах дает теория Галуа (см. $\S 3$ ).

Для определения остальных классов нам понадобится список основных элементарных функций. В этот список, в сущности, входят те функции, которые мы проходили в школе и которые часто вносят в клавиатуры калькуляторов.

Список основных элементарных функций.

1) Все комплексные константы и независимая переменная $x$.

2) Экспонента, логарифм и степенная функция $x^{\alpha}$, где $\alpha$-любая комплексная константа.

3) Тригонометрические функции: синус, косинус, тангенс, котангенс.

4) Обратные тригонометрические функщии: арксинус, арккосинус, арктангенс, арккотангенс.

Перейдем теперь к списку классических операций над функциями. Здесь приводится начало списка. Он будет продолжен в п. 2.1.

Список классических операций. 1) Операщия суперпозищии, сопоставляюшая функщиям $f, g$ функцию $f \circ g$. 
2) Арифметические операции, сопоставляющие функциям $f$ и $g$ функции $f+g$, $f-g, f g$ и $f / g$.

3) Операция дифференцирования, сопоставляющая функции $f$ функцию $f^{\prime}$.

4) Операция интегрирования, сопоставляюшая функции $f$ ее неопределенньй интеграл $y$ (т.е. любую функцию $y$ такую, что $y^{\prime}=f$; по функции $f$ функция $y$ определена с точностью до аддитивной постоянной).

5) Операчия решения алгебрачческого уравнения, сопоставляющая функциям $f_{1}, \ldots, f_{n}$ функцию $y$ такую, что $y^{n}+f_{1} y^{n-1}+\cdots+f_{n}=0$ (по функциям $f_{1}, \ldots, f_{n}$ функция $y$ определена не вполне однозначно, так как алгебраическое уравнение степени $n$ может иметь $n$ решений).

Вернемся теперь к определению классических классов функций одной переменной.

Элементарные функции одной переменной.

Список основных функиии: основные элементарные функции.

Список допустимых операций: суперпозиции, арифметические операции, дифференщирование.

Элементарные функции записываются формулами, например следующей:

$$
f(x)=\operatorname{arctg}(\exp (\sin x)+\cos x) .
$$

Функции одной переменной, представимые в квадратурах.

Список основных функиий: основные элементарные функции.

Список допустимых операчий: суперпозиции, арифметические операции, дифференцирование, интегрирование.

Например, эллиптический интеграл

$$
f(x)=\int_{x_{0}}^{x} \frac{d t}{\sqrt{P(t)}},
$$

где $P$ - кубический полином, представим в квадратурах. Но, как доказал Лиувилль, если полином $P$ не имеет кратных корней, то функция $f$ не является элементарной.

Обобщенные элементарные функции одной переменной. Этот класс функший определяется в точности так же, как класс элементарных функций. Нужно лишш к списку допустимых операций добавить операцию решения алгебраических уравнений.

Функции одной переменной, представимые в обобщенных квадратурах. Этот класс функций определяется в точности так же, как класс функций, представимых в квадратурах. Нужно лишш к списку допустимых операций добавить операцию решения алгебраических уравнений.

Определим еше два класса функщий, близких к классическим классам.

Функции одной переменной, представимые в $k$-радикалах. Этот класс функций определяется в точности так же, как класс функций, представимых в радикалах. Нужно лишш к списку допустимых операций добавить операцию решения алгебраических уравнений степени $\leqslant k$.

Функции одной переменной, представимые в $k$-квадратурах. Этот класс функций определяется в точности так же, как класс функций, представимых в квадратурах. Нужно лиш к списку допустимых операций добавить операцию решения алгебраических уравнений степени $\leqslant k$. 


\section{§. Теория Лиувилля}

Первые строгие доказательства неразрешимости некоторых уравнений в квадратурах и в элементарных функциях были получены в середине девятнадщатого века Лиувиллем (см. [30]-[36)]). Согласно его теории, “достаточно простые” уравнения либо имеют “достаточно простые” решения, либо вообше не решаются в явном виде. Теория Лиувилля, например, отвечает на следуюшие вопросы:

1) при каких условиях неопределенньй интеграл от элементарной функции является элементарной функцией?

2) при каких условиях все решения линейного дифференциального уравнения представимы в обобщенных квадратурах?

Более полно на второй вопрос отвечает дифференциальная теория Галуа (см. $\S 4)$. В настояшем параграфе мы обсудим, как Лиувилль алгебраизировал задачу о разрешимости, и сформулируем некоторые результаты его теории.

2.1. Новые определения классических классов функций. Лиувилль алгебраизировал задачу о разрешимости в элементарных функциях и квадратурах. Главным препятствием на этом пути является абсолютно неалгебраическая операция суперпозиции. Лиувилль обошел это препятствие следуюшим образом: с каждой функцией $g$ из списка основных функций он связал операцию суперпозищии с этой функцией, переводящую функцию $f$, к которой применяется эта операция, в функцию $g \circ f$. Лиувилль заметил, что все основные элементарные функщии сводятся к логарифму и к экспоненте (см. лемму 2.1 ниже). Суперпозиции $y=\exp f$ и $z=\ln f$ можно рассматривать как решения уравнений $y^{\prime}=f^{\prime} y$ и $z^{\prime}=f^{\prime} / f$. Таким образом, внутри классических классов функций вместо абсолютно неалгебраической операции суперпозиции достаточно рассматривать операции решения простых дифференциальных уравнений. После этого задача о разрешимости в классических классах функций становится дифференциально-алгебраической и переносится на абстрактные дифференциальные поля. Приступим к реализации этой программы.

Продолжим список классических операций.

Список классических операций (начало списка в п. 1.2).

6) Операция взятия әкспоненты, сопоставляющая функции $f$ функцию $\exp f$.

7) Операция взятия логарифма, сопоставляющая функции $f$ функцию $\ln f$.

Приведем теперь новые определения трансцендентных классических классов функщий.

Элементарные функции одной переменной.

Список основных функиий: все комплексные константы и независимая переменная $x$.

Список допустимых операций: взятие экспоненты, взятие логарифма, арифметические операции, дифференцирование.

Функции одной переменной, представимые в квадратурах.

Список основных функиий: все комплексные константы.

Cnисок допустимых операчий: взятие экспоненты, арифметические операции, дифференцирование, интегрирование.

Обобщенные элементарные функции и функции, представимые в обобщенных квадратурах и $k$-квадратурах одной переменной, определяются так же, как соответствуюшие необобшенные классы функций, нужно лишь к списку 
допустимых операций добавить соответственно операцию решения алгебраических уравнений или операцию решения алгебраических уравнений степени не вьше $k$.

ЛЕмма 2.1. Основные әлементарные функиии выражаются с помощью комплексных констант, арифметических операций и суперпозиций через әкспоненту и логарифм.

ДОКАЗАТЕльствО. Для степенной функции $x^{\alpha}$ нужное выражение доставляет равенство $x^{\alpha}=\exp (\alpha \ln x)$. Для тригонометрических функций нужные выражения вытекают из формулы Эйлера $e^{a+b i}=e^{a}(\cos b+i \sin b)$. При вешественных значениях $x$ имеем $\sin x=\frac{1}{2 i}\left(e^{i x}-e^{-i x}\right)$ и $\cos x=\frac{1}{2}\left(e^{i x}+e^{-i x}\right)$. В силу аналитичности эти же формулы справедливы и для комплексных $x$. Функции тангенс и котангенс выражаются через синус и косинус. Покажем, что для вешественных $x$ справедливо равенство $\operatorname{arctg} x=\frac{1}{2 i} \ln z$, где $z=\frac{1+i x}{1-i x}$. Очевидно, что $|z|=1, \arg z=2 \arg (1+i x)$, $\operatorname{tg}(\arg (1+i x))=x$, что и доказьвает нужное равенство. В силу аналитичности это же равенство справедливо и для комплексных значений $x$. Остальные обратные тригонометрические функции выражаются через $\operatorname{arctg}$. Именно, $\operatorname{arcctg} x=\frac{\pi}{2}-\operatorname{arctg} x$, $\arcsin x=\operatorname{arctg} \frac{x}{\sqrt{1-x^{2}}}, \arccos x=\frac{\pi}{2}-\arcsin x$. Квадратный корень, участвующий в выражении функции $\arcsin$, выражается через экспоненту и логарифм: $x^{1 / 2}=$ $\exp (1 / 2) \ln x$. Лемма доказана.

ТеОрема 2.2. Для кажсдого из трансцендентных классических классов функчий новое и старое определения (см. настояший параграф̆ и п. 1.2) әквивалентны.

ДокАЗАТЕЛЬСТво. В одну сторону теорема очевидна: ясно, что каждая функция, принадлежашая некоторому классическому классу функций, понимаемому в смысле нового определения, принадлежит тому же классу, понимаемому в смысле старого определения.

Докажем теорему в другую сторону. Согласно лемме 2.1, основные элементарные функции лежат в классах элементарных и обобщенных элементарных функций, понимаемых в смысле нового определения. Из этой же леммы вытекает, что классы функций, представимые в квадратурах, обобшенных квадратурах и $k$-квадратурах, понимаемые в смысле нового определения, тоже содержат основные элементарные функции. Действительно, независимая переменная $x$ принадлежит этим классам, так как она получается интегрированием константы 1 , ибо $x^{\prime}=1$. Вместо операции взятия логарифма, которой нет среди допустимых операций в этих классах, можно использовать операцию интегрирования, так как $(\ln f)^{\prime}=f^{\prime} / f$.

Нам осталось показать, что классические классы функций, понимаемые в смысле нового определения, замкнуты относительно суперпозиций. Дело здесь в следующем: операция взятия суперпозиции коммутирует со всеми операциями, фигурирующими в новых определениях классов функций, за исключением операций дифференцирования и интегрирования. Так, например, результат применения операции ехр к суперпозиции $g \circ f$ совпадает с результатом применения операции взятия суперпозиции к функциям $\exp g$ и $f$, т.е. $\exp (g \circ f)=(\exp g) \circ f$. Аналогично $\ln (g \circ f)=(\ln g) \circ f$, $\left(g_{1} \pm g_{2}\right) \circ f=\left(g_{1} \circ f\right) \pm\left(g_{2} \circ f\right),\left(g_{1} g_{2}\right) \circ f=\left(g_{1} \circ f\right)\left(g_{2} \circ f\right),\left(g_{1} / g_{2}\right) \circ f=\left(g_{1} \circ f\right) /\left(g_{2} \circ f\right)$. 
Если функция $y$ удовлетворяет уравнению $y^{n}+g_{1} y^{n-1}+\cdots+g_{n}=0$, то функция $(y \circ f)$ удовлетворяет уравнению

$$
(y \circ f)^{n}+\left(g_{1} \circ f\right)(y \circ f)^{n-1}+\cdots+\left(g_{n} \circ f\right)=0 .
$$

Для операций дифференщирования и интегрирования имеем следуюшие простые коммутационные соотношения с операцией суперпозиции: $(g)^{\prime} \circ f=(g \circ f)^{\prime}\left(f^{\prime}\right)^{-1}$ (если функция $f$-константа, то функция $(g)^{\prime} \circ f$ - тоже константа) и если $y$-неопределенншй интеграл функции $g$, то $y \circ f$ - неопределенньй интеграл функщии $(g \circ f) f^{\prime}$ (другими словами, интегрированию функции $g$ при суперпозиции с функцией $f$ соответствует интегрирование функции $g \circ f$, домноженной на функцию $\left.f^{\prime}\right)$.

Отсюда и вытекает замкнутость классических классов, понимаемых в смысле нового определения, относительно суперпозиций. Действительно, если функция $g$ получается из констант (или из констант и независимой переменной) при помоши операций, которые мы обсуждали вьше, то функщия $g \circ f$ получается применением тех же или почти тех же операций, как в случае интегрирования и дифференщирования, из функции $f$. Теорема доказана.

ЗАмЕчАниЕ. Легко видеть, что операцию дифференцирования тоже можно исключить из списков допустимых операций классических классов функций. Для доказательства достаточно воспользоваться явным вычислением производных экспоненты и логарифма и правилами дифференщирования формул, включаюших суперпозиции и арифметические операции. Однако исключение операции дифференцирования никак не помогает в задаче о разрешимости уравнений в конечном виде.

2.2. Расширения Лиувилля абстрактных и функциональных дифференциальных полей. Поле $K$ назьвается дифференциальным полем, если задано аддитивное отображение $a \rightarrow a^{\prime}$, удовлетворяющее соотношению Лейбница $(a b)^{\prime}=$ $a^{\prime} b+a b^{\prime}$. Элемент $y$ дифференщиального поля $K$ назьвается константой, если $y^{\prime}=0$. Все константы дифференциального поля образуют подполе, которое назьвается полем констант. Во всех интересующих нас случаях полем констант является поле комплексных чисел. Мьг всегда в дальнейшем предполагаем, что дифференциальное поле имеет нулевую характеристику и алгебраически замкнутое поле констант. Элемент у дифференциального поля назьвается: экспонентой элемента $а$, если $y^{\prime}=a^{\prime} y$; экспонентой интеграла элемента $a$, если $y^{\prime}=а у ;$ логарифмом элемента $а$, если $y^{\prime}=a^{\prime} / a$; интегралом әлемента $a$, если $y^{\prime}=a$.

Пусть дифференциальное поле $K$ и множество $M$ лежат в некотором дифференциальном поле $F$. Присоединением к дифференциальному полю $K$ множества $M$ называется минимальное дифференциальное поле $K\langle M\rangle$, содержашее поле $K$ и множество $M$.

Дифференщиальное поле $F$, содержашее дифференщиальное поле $K$ и имеющее то же поле констант, называется әлементарным расширением поля $K$, если существует цепочка дифференциальных полей $K=F_{1} \subseteq \cdots \subseteq F_{n}=F$ таких, что при каждом $i=1, \ldots, n-1$ поле $F_{i+1}=F_{i}\left\langle x_{i}\right\rangle$ получается присоединением к полю $F_{i}$ элемента $x_{i}$, причем $x_{i}$ - экспонента или логарифм некоторого элемента $a_{i}$ поля $F_{i}$. Элемент $a \in F$ назьвается элементарным над $K, K \subset F$, если он содержится в каком-либо элементарном расширении поля $K$. 
Обобщенное элементарное расширение, расширение Лиувилля, обобщенное расширение Лиувилля и $k$-расширение Лиувилля поля $K$ определяются аналогично. При построении обобщенных элементарных расширений допускаются присоединения экспонент, логарифмов и алгебраические расширения. При построении расширений Лиувилля допускаются присоединения интегралов и экспонент интегралов. В обобщенных расширениях Лиувилля и $k$-расширениях Лиувилля кроме этого допускаются соответственно алгебраические расширения и присоединения решений алгебраических уравнений степени не вьше $k$. Элемент $a \in F$ называется обобщенно-элементарным над $K, K \subset F($ представимым в квадратурах, в обобщенных квадpamypax, в $k$-квадратурах над $K$ ), если $а$ содержится в каком-либо обобщенно-элементарном расширении (расширении Лиувилля, обобшенном расширении Лиувилля, $k$-расширении Лиувилля) поля $K$.

ЗАмЕчАниЕ. Уравнение для экспоненты интеграла проше, чем уравнение для экспоненты. Поэтому при определении расширений Лиувилля и т. д. используются присоединения экспонент интегралов. Вместо этого можно было бы отдельно присоединять экспоненты и отдельно интегралы.

Перейдем к функциональньм дифференциальным полям. Именно с такими полями мы будем иметь дело в обзоре (хотя некоторые результаты без труда переносятся на абстрактные дифференциальные поля).

Всякое подполе $K$ поля всех мероморфных функций в связной области $U$ на сфере Римана, содержашее все комплексные константы и замкнутое относительно дифференцирования (т.е. если $f \in K$, то $f^{\prime} \in K$ ), доставляет пример функционального дифференциального поля. Дадим теперь общее определение. Пусть $V, v$ - пара, состоящая из связной римановой поверхности $V$ и мероморфного векторного поля $v$ на ней. Производная Ли $L_{v}$ вдоль векторного поля $v$ действует на поле $F$ всех мероморфных функций на поверхности $V$ и задает дифференцирование $f^{\prime}=L_{v} f$ в этом поле. Функчиональное дифференииальное поле - это любое дифференщиальное подполе поля $F$, содержашее все комплексные константы.

Иногда удобнее использовать другое определение дифференцирования поля функций, в котором вместо мероморфного векторного поля фигурирует мероморфная 1-форма $\alpha$. Производную $f^{\prime}$ функции $f$ можно определить следующей формулой: $f^{\prime}=d f / \alpha$ (частное двух мероморфных 1-форм является корректно определенной мероморфной функцией). Описанное дифференцирование - это производная Ли $L_{v}$ вдоль векторного поля $v$, связанного с формой $\alpha$ следуюшим соотношением: значение формы $\alpha$ на поле $v$ тождественно равно единице.

Для расширения функциональных полей полезна следующая конструкция. Пусть $K$ - некоторое подполе поля мероморфных функций на связной римановой поверхности $V$, снабженной мероморфной формой $\alpha$, инвариантное относительно дифференцирования $f^{\prime}=d f / \alpha$ (т.е. если $f \in K$, то $f^{\prime} \in K$ ). Рассмотрим любую связную риманову поверхность $W$ вместе с аналитическим отображением $\pi: W \rightarrow V$. Фиксируем на $W$ форму $\beta=\pi^{*} \alpha$. Дифференциальное поле $F$ всех мероморфных функций на $W$ с дифференцированием $\varphi^{\prime}=d \varphi / \beta$ содержит дифференциальное подполе $\pi^{*} K$, состоящее из функщий вида $\pi^{*} f$, где $f \in K$. Дифференщиальное поле $\pi^{*} K$ изоморфно дифференщиальному полю $K$, и оно лежит внутри дифференщиального поля $F$. Если 
удачно подобрать поверхность $W$, то расширение поля $\pi^{*} K$, изоморфного полю $K$, можно произвести внутри поля $F$.

Пусть требуется расширить поле $K$, скажем, интегралом $y$ некоторой функции $f \in$ $K$. Это можно сделать следующим образом. Над римановой поверхностью $V$ можно рассмотреть риманову поверхность $W$ неопределенного интеграла $y$ формы $f \alpha$. По самому определению римановой поверхности $W$ существует естественная проекция $\pi: W \rightarrow V$ и функция $y$ является однозначной мероморфной функцией на поверхности $W$. Дифференциальное поле $F$ мероморфных функций на $W$ с операцией дифференщирования $f^{\prime}=d f / \pi^{*} \alpha$ содержит как элемент $y$, так и поле $\pi^{*} K$, изоморфное полю $K$. Поэтому расширение $\pi^{*} K\langle y\rangle$ определено и является подполем дифференциального поля $F$. Именно эту конструкцию расширения мы имеем в виду, когда говорим о расширениях функциональных дифференциальных полей. Эта же конструкция позволяет присоединить к функциональному дифференциальному полю $K$ логарифм, экспоненту, интеграл или экспоненту интеграла от любой функции $f$ из поля $K$. Для любых функций $f_{1}, \ldots, f_{n} \in K$ можно таким способом присоединить к $K$ решение $y$ алгебраического уравнения $y^{n}+f_{1} y^{n-1}+\cdots+f_{n}=0$ или все решения $y_{1}, \ldots, y_{n}$ этого уравнения (присоединение всех решений $y_{1}, \ldots, y_{n}$ можно осушествить на римановой поверхности вектор-функции $\left.\mathbf{y}=\left(y_{1}, \ldots, y_{n}\right)\right)$. Таким же способом для любых функщий $f_{1}, \ldots, f_{n+1} \in K$ можно присоединить к $K n$-мерное аффинное пространство над $\mathbb{C}$ всех решений линейного дифференциального уравнения $y^{(n)}+f_{1} y^{(n-1)}+\cdots+f_{n} y+f_{n+1}=0$. (Напомним, что росток любого решения линейного дифференщиального уравнения аналитически продолжается вдоль кривой на поверхности $V$, не проходящей через полюсы функций $f_{1}, \ldots, f_{n+1}$.)

Итак, все упомянутые выше расширения функчиональных дифференциальных полей можсно осуществить, не выходя из класса функциональных дифференииальных полей. Говоря о расширениях функциональных дифференциальных полей, мы всегда имеем в виду именно эту процедуру.

Дифференщиальное поле, состояшее из всех комплексных констант, и дифференциальное поле, состоящее из всех рациональных функций от одной переменной, можно рассматривать как дифференциальные поля функций, определенных на сфере Римана.

Сформулируем снова теорему 2.2 , используя определения из абстрактной дифференциальной алгебры и конструкцию расширения функциональных дифференциальных полей.

ТЕОРема 2.2'. Функиия одной комплексной переменной (возможнн, многозначная) принадлежит:

1) классу әлементарных функиий, если и только если она принадлежит некоторому әлементарному расширению поля всех рациональных функиий одной переменной

2) классу обобщенных элементарных функиий, если и только если она принадлежит некоторому обобщенному әлементарному расширению поля рачиональнььх функиий;

3) классу функиий, представимых в квадратурах, если и только если она принадлежит некоторому расширению Лиувилля поля всех комплексньх констант; 
4) классу функиий, представимых в k-квадратурах, если и только если она принадлежст некоторому $k$-расширению Лиувилля поля всех комплексньх констант:

5) классу функиий, представимых в обобщенных квадратурах, если и только если она принадлежит обобщенному расширению Лиувилля поля всех комплексных констант.

2.3. Некоторые результаты теории Лиувилля. В этом пункте мы приведем без доказательства формулировки ряда результатов теории Лиувилля.

2.3.1. Неэлементарность неопределенных интегралов. Когда мы начинаем учить анализ, нас учат интегрировать элементарные функции, и это оказьвается далеко не простым занятием. Как доказал Лиувилль, неопределенный интеграл от элементарной функции обычно не является элементарной функцией.

ТеОрема 2.3 (об интегралах). Неопределенный интеграл у функиии $f$, лежащей в функииональном дифференииальном поле $K$, принадлежит некоторому обобщенно-элементарному расширению этого поля, если и только если этот интеграл представим в виде

$$
y(x)=\int_{x_{0}}^{x} f(t) d t=A_{0}(x)+\sum_{i=1}^{n} \lambda_{i} \ln A_{i}(x),
$$

где $A_{i}$ при $i=0,1, \ldots, n$ - некоторые функции из поля $K$.

Условие (1) из теоремы Лиувилля в дифференциальной форме означает, что элемент $f \in K$ представим в виде

$$
f=A_{0}^{\prime}+\sum_{i=1}^{n} \lambda_{i} \frac{A_{i}^{\prime}}{A_{i}}
$$

где $A_{i}$ при $i=0,1, \ldots, n$ - некоторые элементы поля $K$. В абстрактной дифференциальной алгебре справедлив аналог теоремы Лиувилля [34]. В формулировке абстрактной теоремы в качестве $K$ нужно взять абстрактное дифференциальное поле и воспользоваться условием (1) в дифференциальной форме (2).

СлЕДСТВИЕ 2.4. Неопределенный интеграл у от обобщенной әлементарной функиии $f$ является обобщенной әлементарной функиией, если и только если он представим в виде

$$
y(x)=A_{0}(x)+\sum_{i=1}^{n} \lambda_{i} \ln A_{i}(x),
$$

где $A_{i}$ при $i=0,1, \ldots, n$ - рациональные функции с комплексными коэффициентами от функиии $f$ и ее производных.

Априори, интеграл от элементарной функщии $f$ мог бы быть очень сложной элементарной функцией. Теорема Лиувилля показывает, что ничего такого случиться не может. Или интеграл от элементарной функции неэлементарен, или он имеет описанньй в следствии простой вид. 
Берется ли интеграл от алгебраической функции в явном виде? Этому вопросу были посвяшены пионерские работы Абеля, заложившие основы теории алгебраических кривых и абелевых интегралов и вдохновившие Лиувилля на создание его теории. Грубо говоря, ответ на этот вопрос такой. Если риманова поверхность алгебраической функции имеет нулевой род, то ее интеграл всегда берется в обобщенных элементарных функциях. Если же род римановой поверхности положителен, то интеграл, как правило, не элементарен и берется в обобщенных элементарных функциях в исключительных случаях. Более подробньй ответ дает формулируемая ниже теорема Лиувилля об абелевых интегралах.

ТЕОРема 2.5 (об абелевых интегралах). Неопределенный интеграл у от алгебраической функиии А комплексной переменной $x$ берется в обобщенных әлементарных функииях, если и только если он представим в виде

$$
y(x)=\int_{x_{0}}^{x} A(t) d t=A_{0}(x)+\sum_{i=1}^{k} \lambda_{i} \ln A_{i}(x),
$$

где $A_{i}$ при $i=0,1, \ldots, k$ - алгебраические функиии, однозначные на римановой поверхности $W$ подынтегральной функиии $A$.

ДокАЗАТЕльство. Теорема вытекает из теоремы Лиувилля об интегралах элементарных функций, примененной к полю $F$ всех мероморфных функций на поверхности $W$, снабженному следующим дифференцированием: $f^{\prime}=d f / \alpha$, где $\alpha=\pi^{*} d x$ и $\pi: W \rightarrow \bar{C}$ - естественная проекция римановой поверхности функции $A$ на сферу Римана $\bar{C}$ комплексного переменного $x$.

ЗАмечАниЕ. Теорема Лиувилля об абелевых интегралах восходит к Абелю. Абель рассматривал более частную задачу о представимости абелевых интегралов в виде рациональных функций от алгебраических функщий и их логарифмов и пришел к аналогичньм вьводам.

Сформулируем критерий Лиувилля интегрируемости в явном виде функций экспоненщиального типа и приведем примеры неберущихся интегралов.

КРИТЕРИй ЛИУВИЛля. Рассмотрим неопределенный интеграл вида

$$
I(x)=\int_{x_{0}}^{x} f(t) e^{g(t)} d t
$$

где $f$ и $g$-рациональные функиии, причем функиия $g$ непостоянная, а $f$ не обращается в тождественньй нуль. Если существует рациональная функиия а такая, что $a^{\prime}+a g^{\prime}=f$, то $I=a e^{g}+c$, где с-комплексная константа. Если же уравнение $a^{\prime}+a g^{\prime}=f$ неразрешимо в рациональных функциях, то интеграл I не является обобщенной әлементарной функцией.

ПРИМЕРЫ НЕБЕРУШИХСя ИНТЕГРАЛОв. Неопределенные интеграль $\int e^{t^{2}} d t$, $\int_{\mathcal{M}} \frac{e^{t}}{t} d t, \int \frac{d t}{\ln t}, \int \frac{\sin t}{t} d t$ не являются обобщенными әлементарными функиия-

2.3.2. Критерий Лиувилля-Мордухай-Болтовского. Лиувиллю принадлежит первьй результат о неразрешимости линейных дифференщиальных уравнений в явном виде (см. [32], [33]). 
ТеОРема 2.6 (Лиувилль). Уравнение $y^{\prime \prime}+p y^{\prime}+q y=0$ с коэффициентами из функиионального дифференциального поля $K$, все әлементы которого представимы в обобщенных квадратурах, решается в обобщенных квадратурах, если и

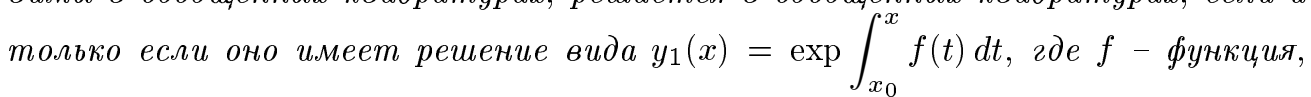
удовлетворяющая алгебраическому уравнению с коэффичиентами в поле $K$.

В одну сторону теорема очевидна. Если известно одно решение $y_{1}$ линейного дифференциального уравнения второго порядка, то его можно решить, понизив порядок уравнения. Доказать теорему в другую сторону достаточно трудно.

Потребовалось более полувека, чтобы обобшить теорему Лиувилля на уравнения $n$-го порядка. Мордухай-Болтовский доказал в 1910 году методом Лиувилля следующий критерий, позволяюший сводить вопрос о разрешимости уравнения к вопросу о разрешимости другого уравнения меньшего порядка.

КРИТЕРИЙ ЛИУВИЛЛЯ-МОРДУХАЙ-БОЛТОВСКОГО. Уравнение $n$-го порядка

$$
y^{(n)}+p_{1} y^{(n-1)}+\cdots+p_{n} y=0
$$

с коэффициентами из функционального дифференциального поля $K$, все әлементы которого представимы в обобщенных квадратурах, решается в обобщенных квадратурах, если и только если оно, во-первых, имеет решение вида $y_{1}(x)=$ $\exp \int_{x_{0}}^{x} f(t) d t$, где $f$ - функиия, лежсащая в некотором алгебраическом расширении $K_{1}$ поля $K$, , во-вторых, если дифференциальное уравнение $(n-1)$-го порядка на функиию $z=y^{\prime}-\frac{y_{1}^{\prime}}{y_{1}} y$ с коэффичиентами из поля $K_{1}$, полученное из исходного уравнения процедурой понижения порядка (см. n. 4.1.2), решается в обобщенных квадратурах над полем $K_{1}$.

В этом же 1910 году появилась теорема Пикара-Вессио, в которой вопрос о разрешимости линейных дифференциальных уравнений решается абсолютно по-другому, с точки зрения дифференциальной теории Галуа.

Ниже мы обсудим основные положения этой теории. Критерий Лиувилля-Мордухай-Болтовского, по существу, эквивалентен теореме Пикара-Вессио. Теория Пикара-Вессио не только объясняет этот критерий, но и дает возможность довести его до явного алгоритма, позволяющего для уравнения с коэффициентами из поля рациональных функщий (имеющих рациональные коэффищиенты) определить, разрешимо уравнение в обобшенных квадратурах или нет (см. [37] и п. 4.7).

\section{§ 3. Разрешимость алгебраических уравнений в радикалах и теория Галуа}

Решается ли заданное алгебраическое уравнение в радикалах? Можно ли решать заданное алгебраическое уравнение степени $n$, используя решение вспомогательных алгебраических уравнений меньшей степени и радикалы? В этом параграфе мы обсуждаем, как теория Галуа решает эти вопросы. Мы обрашаем главное внимание именно на вопросы разрешимости и неразрешимости и приводим без доказательства основную теорему теории Галуа. Без доказательства используются также хорошо 
известные свойства разрешимых групп и групшы $S(k)$. В п. 3.4.1 доказьвается значительно менее известное характеристическое свойство подгрупп групшы $S(k)$. Эти факты из теории групп применяются как в обычной теории Галуа, так и в ее дифференциальном и топологическом вариантах.

"Разрешительная" часть теории Галуа (см. п. 3.3.2), позволяющая решать уравнение в радикалах, весьма проста. Она не использует ни основную теорему теории Галуа, ни вообще теорию полей и относится, по существу, к линейной алгебре. Только эти линейно-алгебраические соображения применяются в топологическом варианте теории Галуа при обсуждении вопроса о представимости алгебраических функций в радикалах. (Однако достаточное условие разрешимости уравнения с помошюю решения вспомогательных уравнений меньшей степени и радикалов опирается не только на линейную алгебру, но и на основную теорему теории Галуа.)

Приведенные выше задачи о разрешимости алгебраических уравнений по своей природе являются чисто алгебраическими и могут быть поставлены над любьм полем $K$. $B$ этом параграфе мы будем предполагать, что поле $K$ имеет нулевую характеристику и содержит все корни из единицы. Этот случай немного проще общего, а для нас основной интерес представляют функциональные дифференциальные поля, которые содержат все комплексные константы.

3.1. Группа Галуа алгебраического уравнения. Рассмотрим некоторое алгебраическое уравнение

$$
a_{n} x^{n}+\cdots+a_{1} x+a_{0}=0
$$

с коэффициентами из поля $K$. Мы считаем, что поле $K$ вложено в некоторое большее поле, содержащее все корни уравнения. Соотношением, определенным над полем $K$, между корнями уравнения (3) называется любой полином $Q$, принадлежащий кольцу $K\left[x_{1}, \ldots, x_{n}\right]$, который обрашается в нуль в точке $\left(x_{1}^{0}, \ldots, x_{n}^{0}\right)$, где $x_{1}^{0}, \ldots, x_{n}^{0}$ - упорядоченньй набор корней уравнения (3).

ОПРЕДЕЛЕНИЕ. Группой Галуа алгебраического уравнения (3) над полем $K$ называется подгрупа $G$ групы $S(n)$ всех перестановок корней уравнения, сохраняюших все соотношения между корнями, определенные над полем $K$ (т.е. если перестановка $\sigma \in S(n)$ принадлежит группе Галуа $G$, то полином $\sigma Q$, полученньй из соотношения $Q$ перестановкой $\sigma$ переменных $x_{1}, \ldots, x_{n}$, тоже обрашается в нуль в точке $\left.\left(x_{1}^{0}, \ldots, x_{n}^{0}\right)\right)$.

ОПРЕДЕЛЕНИЕ. Поле $P$ назьвается расширением Галуа поля $K$, если существует алгебраическое уравнение (3) с коэффициентами в поле $K$ такое, что поле $P$ получается присоединением к полю $K$ всех корней этого уравнения. Группой Галуа расширения Галуа $P$ над полем $K$ назьвается групша всех автоморфизмов поля $P$, оставляющих на месте все элементы поля $K$.

Каждый элемент $\sigma$ из групшы Галуа поля $P$ над полем $K$ переставляет корни уравнения (3) и сохраняет все соотношения между ними, определенные надполем $K$. Таким образом, группа Галуа поля $P$ над $K$ имеет представление в группе Галуа $G$ уравнения (3), определяющего расширение $P$. Очевидно, что это представление является изоморфизмом груп, т.е. група Галуа уравнения и группа Галуа заданного им расширения изоморфны. 
Для доказательства неразрешимости уравнений нам понадобятся следуюшие простые "оценки сверху" групп Галуа.

Лемма 3.1. Группа Галуа уравнения $x^{n}-a=0$ над полем $K, a \in K$, является подгруппой ииклической группы из $n$ әлементов.

ДОКАЗАТЕЛЬСТвО. Фиксируем любой корень $x_{0}$ уравнения $x^{n}-a=0$ и любой первообразньй корень $\xi$ степени $n$ из единицы. Занумеруем корни уравнения $x^{n}-a=0$ вычетами $i$ по модулю $n$, положив $x_{i}$ равным $\xi^{i} x_{0}$. Пусть преобразование $g$ из групшы Галуа переводит корень $x_{0}$ в корень $x_{i}$. Тогда $g\left(x_{k}\right)=g\left(\xi^{k} x_{0}\right)=\xi^{k+i} x_{0}=x_{k+i}$. То есть всякое преобразование групп Галуа задает циклическую перестановку корней.

Следующая лемма непосредственно вытекает из определения групшы Галуа алгебраического уравнения.

Лемма 3.2. Группа Галуа уравнения степени $m \leqslant k$ изоморфна подгруппе әруппь $S(k)$.

3.2. Основная теорема теории Галуа. Пусть $P$ - расширение Галуа поля $K$ и $G$ - его группа Галуа.

Определены следующие отображения между множеством полей, лежаших между $K$ и $P$, и множеством подгрупп групшы Галуа $G$.

1) Oтображение $F d$, сопоставляющее каждой подгруппе $\Gamma$ группы $G$ поле $F d(\Gamma)$, состоящее из элементов поля $P$, остающихся неподвижньми при действии подгруппы $\Gamma$ (ясно, что $K \subseteq F d(\Gamma))$.

2) Отображсение $G p$, сопоставляюшее каждому промежуточному полю $F, K \subseteq$ $F \subseteq P$, подгрупу $G p(F) \subseteq G$, являюшуюся группой Галуа расширения Галуа $P$ поля $F$ ( $P$ является расширением Галуа поля $K$, и поэтому оно автоматически является расширением Галуа промежуточного поля $F, K \subseteq F \subseteq P$ ).

Отображения $F d$ и $G p$ устанавливают соответствие Галуа между подгруппами групшы Галуа и промежуточньми полями расширения Галуа.

Приведем без доказательства следуюшую теорему.

Основная теОРема 3.3 (теории Галуа). Для всякого расширения Галуа P поля $K$ с әруппой Галуа $G$

1) композииия отображений $F d$ и Gp является тождественныцм отображением множества промежуточных полей в себя: если $F$ - поле и $K \subseteq F \subseteq P, \operatorname{mo} F d(G p(F))=F$

2) композиция отображений $G p$ и $F d$ является тождественныцм отображением множества подгрупп группы Галуа в себя: если Г - подгруппа әруппь Галуа, $\Gamma \subset G, \operatorname{mo} G p(F d(\Gamma))=\Gamma$;

3) промежуточное поле $F, K \subseteq F \subseteq P$, является расширением Галуа поля $K$, если и только если группа $G p(F)$ является нормальнылм делителем группь $G$; при этом группа Галуа расширения Галуа $F$ поля $K$ является фактор-группой группь $G$ по нормальному делителю $G p(F)$.

Поле $P$ является расширением Галуа поля $K$, если и только если существует конечная группа Г автоморфизмов поля $P$ такая, ито она оставляет неподвижными все әлементы поля $K$ и только их. 
Что произойдет с группой Галуа уравнения, если поле коэффициентов $K$ расширить, заменив его большим полем $K_{1}$ ? Этот вопрос особенно интересен в том случае, когда поле $K_{1}$ является расширением Галуа поля $K$. Обозначим через $G_{1}$ группу Галуа расширения $K_{1}$ поля $K$. Результаты о неразрешимости алгебраических уравнений основаны на следующей теореме.

ТЕОРема 3.4 (об изменении групшы Галуа уравнения при расширении Галуа поля коэффициентов). При замене поля коэффичиентов $K$ его расширением Галуа $K_{1}$ группа Галуа $G$ уравнения заменяется некоторым своим нормальным делителем $H$. Фактор-группа $G / H$ группь $G$ относительно әтого нормального делителя изоморфна некоторой фактор-группе группь Галуа $G_{1}$ нового поля коәффичиентов $K_{1}$ над старым полем коэффичиентов $K$.

ДокАЗАТЕЛЬСтво. Пусть $Q$ - наименьшее расширение Галуа поля $K$, содержащее расширения $P$ и $K_{1}$ поля $K$. (Расширение $Q$ получается присоединением к $K$ всех корней произведения полиномиальных уравнений, определяюших расширения Галуа $P$ и $K_{1}$ поля $K$.) Поле $K$ неподвижно при действии группы Галуа $\Gamma_{Q}$ расширения $Q$, а поля $K_{1}$ и $P$ инвариантны относительно этого действия. Следовательно, поле $K_{1} \cap P$ инвариантно относительно действия группы $\Gamma_{Q}$ и является поэтому расширением Галуа поля $K$.

Групша Галуа $H$ расширения Галуа $P$ поля $K_{1} \cap P$ является нормальным делителем групшы Галуа расширения Галуа $P$ поля $K$, так как $K_{1} \cap P$ - расширение Галуа поля $K$ и $K_{1} \cap P \subset P$. С другой стороны, группа $H$ является фактор-группой групшы Галуа расширения Галуа $K_{1}$ поля $K$, поскольку $K_{1} \cap P$ - расширение Галуа поля $K$ и $K_{1} \cap P \subset K_{1}$.

Осталось показать, что группа Галуа $H$ поля $P$ над $K_{1} \cap P$ совпадает с групой Галуа Г поля $Q$ над $K_{1}$. Действительно, группа $\Gamma$ является подгруппой в $H$, так как каждое соотношение над полем $K_{1} \cap P$ является, в частности, соотношением между корнями того же уравнения над полем $K_{1}$. Пусть $\Gamma \neq H$. Согласно основной теореме, примененной к расширению $P$ поля $K$, поле инвариантов $F$ относительно действия $\Gamma$ на $P$ строго больше, чем $K_{1} \cap P$. С другой стороны, все элементы поля $F$ лежат как в поле $P$, так и в поле $K_{1}$. Противоречие доказьвает, что $\Gamma=H$.

3.3. Разрешимость в радикалах. Решается ли заданное алгебраическое уравнение в радикалах? Теория Галуа была создана для ответа на этот вопрос. Начнем с формального определения.

Алгебраическое уравнение над полем $K$ разрешимо в радикалах, если существует цепочка расширений $K=K_{0} \subset \cdots \subset K_{N}$, в которой поле $K_{i+1}$ получается из поля $K_{i}, i=0,1, \ldots, N-1$, присоединением радикала, а поле $K_{N}$ содержит все корни исходного алгебраического уравнения.

Теорема 3.5. Алгебраическое уравнение решается в радикалах, если и только если его группа Галуа разрешима.

ДокАЗАТЕльство. Достаточное условие разрешимости групшы Галуа для разрешимости уравнения в радикалах мы докажем ниже в п. 3.3.1 (см. следствие 3.10). Покажем, что если алгебраическое уравнение решается в радикалах, то его группа Галуа разрешима. Проследим, что происходит с группой Галуа уравнения при переходе 
от поля коэффициентов $K_{i}$ к полю коэффициентов $K_{i+1}$. Обозначим через $G_{i}$ группу Галуа нашего уравнения над полем $K_{i}$. Тогда, согласно теореме 3.4 , группа $G_{i+1}$ является нормальной подгруппой в групе $G_{i}$, причем фактор-группа $G_{i} / G_{i+1}$ является одновременно фактор-группой групшы Галуа поля $K_{i+1}$ над полем $K_{i}$. Так как поле $K_{i+1}$ получается из поля $K_{i}$ присоединением радикала, то согласно лемме 3.1 группа Галуа поля $K_{i+1}$ над полем $K_{i}$ коммутативна. Так как все корни алгебраического уравнения по условию лежат в поле $K_{N}$, то група Галуа $G_{N}$ алгебраического уравнения над полем $K_{N}$ тривиальна. Итак, у групш Галуа $G$ сушествует цепочка подгрупп $G=G_{0} \supset \cdots \supset G_{N}$, в которой группа $G_{i+1}$ является нормальным делителем группы $G_{i}$ с коммутативной фактор-группой $G_{i} / G_{i+1}$, а группа $G_{N}$ тривиальна. То есть групша Галуа $G$ разрешима.

3.3.1. Достаточное условие разрешимостив радикалах. Пусть $K$ - подполе поля $P$, и пусть на $P$ действует конечная группа автоморфизмов $G$, оставляющая неподвижными все элементы поля $K$ и только их. Если поле $P$ получено из поля $K$ присоединением всех корней алгебраического уравнения над полем $K$, то такая група автоморфизмов $G$ сушествует (и изоморфна групе Галуа уравнения). Существование групшы $G$ никак не самоочевидно и представляет собой один из центральных фактов теории Галуа (см. п. 3.2). Однако в ряде важных случаев группа $G$ задана априори. Так, например, обстоит дело, если $K$ - поле рациональных функций одной переменной, $P$ - поле, полученное присоединением к $K$ всех ветвей алгебраической функции, и $G$ - монодромии этой алгебраический функции (см. п. 5.1.2). Другой пример доставляет общее алгебраическое уравнение степени $n$ (см. п. 3.3.3).

Пусть в рассматриваемой ситуации группа $G$ разрешима. Тогда любой элемент поля $P$ представим в радикалах через элементы поля $K$. Конструкция представления элемента в радикалах, в основном, относится к линейной алгебре. Она очень слабо использует то обстоятельство, что мы имеем дело с полями. Чтобы подчеркнуть это, мы опишем эту конструкцию, взяв вместо поля алгебру $V$, которая может быть и некоммутативной. В дальнейшем нам даже не понадобится перемножать разные элементы этой алгебры. Мы будем использовать лишь операцию возведения в целую неотрицательную степень $k$ и однородность этой операции относительно умножения на элементы основного поля $(\lambda a)^{k}=\lambda^{k} a^{k}$ при $a \in V, \lambda \in K$. Итак, пусть $V$ - алгебра над полем нулевой характеристики $K$, содержащем все корни из единицы.

УТВЕРЖДЕНИЕ 3.6. Пусть $A$ - автоморфизм конечного порядка $n$ алгебры $V$, $A^{n}=I$ и $V_{0}-$ подалгебра инвариантов. Пусть поле нулевой характеристики $K$ содержит все корни степени п из единищы. Тогда каждый элемент $x$ алгебры $V$ представим в виде суммы $x=x_{1}+\cdots+x_{n}$ әлементов $x_{i} \in V \operatorname{ma\kappa их,~что~} x_{i}^{n} \in V_{0}$.

ДокАЗАтельство. Рассмотрим конечномерное линейное пространство в алгебpe $V$, натянутое на орбиту $x, A(x), \ldots, A^{n-1}(x)$ элемента $x$ относительно действия автоморфизма $A$ и его степеней. Так как $A^{n}$ по условию является тождественньм преобразованием пространства $V$ и поле $K$ содержит все собственные числа линейного преобразования $A: V \rightarrow V$, то $V$ раскладывается в прямую сумму собственных подпространств $V=V_{1} \oplus \cdots \oplus V_{n}$ оператора $A$ с собственньми числами $\xi_{k}, 1 \leqslant k \leqslant n,-$ корнями $n$-й степени из единищы (некоторые из пространств $V_{i}$ могут быть нулевыми). Поэтому вектор $x$ представим в виде $x=x_{1}+\cdots+x_{n}$, где $A\left(x_{k}\right)=\xi_{k} x_{k}$. Следова- 
тельно, $A\left(x_{k}^{n}\right)=\left(A\left(x_{k}\right)\right)^{n}=\xi_{k}^{n} x_{k}^{n}=x_{k}^{n}$. То есть элемент $x_{k}^{n}$ принадлежит алгебре инвариантов автоморфизма $A$.

Введем следующее определение.

ОПредЕлЕнИЕ. Скажем, что элемент $x$ алгебры $V$ получается операцией извлечения $n$-й степени из әлемента $a$, если вьполняется равенство $x^{n}=a$.

Пользуясь этим определением, утверждение 3.6 можно интерпретировать следуюшим образом: каждый элемент $x$ в алгебре $V$ представляется в виде суммы корней $n$-й степени из элементов алгебры инвариантов. Утверждение 3.6 легко обобщается на случай действия конечной коммутативной группы автоморфизмов.

УТВЕРЖДЕНИЕ 3.7. Пусть $G$ - конечная коммутативная группа порядка $n$ автоморфизмов алгебры $V$, и пусть $V_{0}-$ подалгебра инвариантов. Тогда каждый әлемент $x$ алгебры $V$ представим в виде суммы $x=x_{1}+\cdots+x_{n}$ әлементов $x_{i} \in V$ таких, что $x_{i}^{m} \in V_{0}$, где $m$ - наименьшее общее кратное порядков әлементов группь $G$.

Доказательство утверждения 3.7 почти дословно повторяет доказательства утверждения 3.6 , нужно только воспользоваться тем, что конечная коммутативная группа линейных преобразований в некотором базисе приводится к диагональному виду.

ТЕОРема 3.8. Пусть $G$ - конечная разрешимая группа автоморфизмов алгебры $V$, и пусть $V_{0}$ - подалгебра инвариантов. Тогда каждыи әлемент х алгебры $V$ получается из әлементов алгебры инвариантов $V_{0}$ при помощи извлечения корней и суммирований.

Докажем сначала следующее простое утверждение о действии групы на множестве.

Пусть группа $G$ действует на множестве $X$, пусть $H$ - нормальньй делитель группы $G$, и пусть $X_{0}$ - подмножество $X$, состоящее из неподвижных точек относительно действия групшы $G$.

УТВЕРЖДЕНИЕ 3.9. Подмножество $X_{H}$ множества $X$, состоящее из неподвижных точек относительно действия нормального делителя $H$, инвариантно относительно действия группь $G$. На множестве $X_{H}$ естественно действует фактор-группа $G / H$ с неподвижным множеством $X_{0}$.

ДокАЗАТЕЛьСтво. Пусть $g \in G$ и $h \in H$. Тогда элемент $g^{-1} h g$ принадлежит нормальному делителю $H$. Пусть $x \in X_{H}$. Тогда $g^{-1} h g(x)=x$, или $h(g x)=g(x)$, что означает, что элемент $g \in X$ неподвижен при действии нормального делителя $H$. Итак, множество $X_{H}$ инвариантно относительно действия групшы $G$. При этом действии элементам нормального делителя $H$ соответствуют тождественные преобразования. Поэтому действие группы $G$ на $X_{H}$ сводится к действию фактор-группы $G / H$.

Перейдем теперь к доказательству теоремы 3.8 .

ДокАЗАтельство. Так как группа $G$ разрешима, то у нее сушествует цепочка вложенных подгруп $G=G_{0} \supset \cdots \supset G_{m}=e$ такая, что група $G_{m}$ совпадает с 
единичньм элементом $е$ и при $i=1, \ldots, m$ группа $G_{i}$ является нормальным делителем группы $G_{i-1}$, причем фактор-группа $G_{i-1} / G_{i}$ коммутативна.

Такая цепочка подгрупп существует ввиду разрешимости группы $G$.

Обозначим через $V_{0} \subset \cdots \subset V_{m}=V$ цепочку подалгебр инвариантов алгебры $V$ относительно действия груп $G_{0}, \ldots, G_{m}$. Согласно утверждению 3.9, коммутативная фактор-группа $G_{i-1} / G_{i}$ естественно действует на алгебры инвариантов $V_{i}$, оставляя неподвижной подалгебру инвариантов $V_{i-1}$. Согласно утверждению 3.7, каждый элемент алгебры $V_{i}$ выражается при помоши суммирования и извлечения корней через элементы алгебры $V_{i-1}$. Последовательно повторяя это рассуждение, мы выразим каждый элемент алгебры $V$ через элементы подалгебры $V_{0}$ цепочкой извлечения корней и суммирования.

ЗАмечАнИЕ. Цепочку нормальных делителей конечной разрешимой групшы $G$ можно выбрать таким образом, что все фактор-группы $G_{i} / G_{i-1}$ будут не только коммутативными, но и циклическими. Поэтому для доказательства теоремы вполне достаточно использовать утверждение 3.6.

Закончим доказательство теоремы 3.5 о разрешимости уравнений в радикалах.

СлЕДСТВИЕ 3.10. Если группа Галуа алгебраического уравнения над полем $K$ разрешима, то уравнение решается в радикалах над әтим полем.

ДокАЗАТЕльство. Согласно основной теореме теории Галуа, поле инвариантов относительно действия группы Галуа совпадает с полем $K$. Поэтому следствие вытекает из теоремы 3.8 .

3.3.2. Решения уравнений второй, третьей и четвертой степени. Теорема 3.8 объясняет, почему уравнения маленькой степени решаются в радикалах.

Пусть $K\left[x_{1}, \ldots, x_{n}\right]$ - кольцо многочленов от переменных $x_{1}, \ldots, x_{n}$ над полем нулевой характеристики $K$, содержашем все корни из единищы. Група $S(n)$ перестановок $n$ элементов действует на этом кольце, переставляя переменные $x_{1}, \ldots, x_{n}$ в многочленах из этого кольца. Алгебра инвариантов $K_{S}\left[x_{1}, \ldots, x_{n}\right]$ относительно этого действия состоит из симметричных многочленов. Каждый многочлен этой алгебры явным образом представляется в виде многочлена от переменных $\sigma_{1}, \ldots, \sigma_{n}$, где $\sigma_{1}=x_{1}+\cdots+x_{n}, \sigma_{2}=\sum_{i<j} x_{i} x_{j}, \ldots, \sigma_{n}=x_{1} \cdots x_{n}$. При $n=2,3,4$ группа $S(n)$ разрешима. Применяя теорему 3.8 , получаем, что каждьй полином от $x_{1}, \ldots, x_{n}$ при $n \leqslant 4$ выражается через основные симметрические многочлены $\sigma_{1}, \ldots, \sigma_{n}$ при помощи извлечения корней, суммирования и умножения на рациональные числа. Рассмотрим алгебраическое уравнение

$$
x^{n}+a_{1} x^{n-1}+\cdots+a_{n}=0 .
$$

Согласно формулам Виета, коэффициенты этого уравнения с точностью до знака совпадают с основными симметрическими функциями от его корней $x_{1}, \ldots, x_{n}$, поэтому теорема 3.8 при $n=2,3,4$ дает явное выражсение корней уравнения (4) через коэффичиенты этого уравнения при помощи извлечения корней, суммирования и умножения на рациональные числа.

3.3.3. Общее алгебраическое уравнение и теорема Абеля. Общим алгебраическим уравнением степени $n$ назьвается алгебраическое уравнение

$$
x^{n}+a_{1} x^{n-1}+\cdots+a_{n}=0
$$


коэфрициенты $a_{1}, \ldots, a_{n}$ которого являются независимьми комплексными переменными. Общее алгебраическое уравнение определено над полем $K$ рациональных функций от переменных $a_{1}, \ldots, a_{n}$. Пусть $a^{0}$ - некоторая точка в пространстве $\mathbb{C}^{n}$ с координатами $a_{1}, \ldots, a_{n}$, в которой дискриминант уравнения (5) не обрашается в нуль. В малой окрестности $U$ точки $a^{0}$ существует $n$ алгебраических функций $x_{1}, \ldots, x_{n}$, удовлетворяюших уравнению (5). Рассмотрим поле $P_{U}$ мероморфных функций в области $U$, порожденное функциями $x_{1}, \ldots, x_{n}$ над полем $K_{U}$, изоморфное полю $K$ и состоящее из ограничений рациональных функций на область $U$. По определению поле $P$ является расширением Галуа поля $K_{U}$, соответствуюшим уравнению $(5)$ над $K_{U}$.

Пусть $V: \mathbb{C}^{n} \rightarrow \mathbb{C}^{n}$ - отображение Виета, переводящее точку $x=\left(x_{1}, \ldots, x_{n}\right)$ в точку $a(x)$ с координатами $a_{1}=-\left(x_{1}+\cdots+x_{n}\right), \ldots, a_{n}=(-1)^{n} x_{1} \cdots x_{n}$. На пространстве-прообразе действует група $S(n)$ перестановок координат $x_{1}, \ldots, x_{n}$. Действие $S(n)$ переносится на поле $R\left[x_{1}, \ldots, x_{n}\right]$ рациональных функций от переменных $x_{1}, \ldots, x_{n}$. Поле инвариантов $R_{S}\left[x_{1}, \ldots, x_{n}\right]$ относительно этого действия состоит из симметричных рациональных функций от $x_{1}, \ldots, x_{n}$.

УТВЕРЖДЕНИЕ 3.11.

1) Пара полей $K_{U} \subset P_{U}$ изоморфна паре полей $R_{S}\left[x_{1}, \ldots, x_{n}\right] \subset R\left[x_{1}, \ldots, x_{n}\right]$.

2) Группа Галуа уравнения (5) изоморфна симметрической группе $S(n)$.

ДокАЗАТЕЛЬСТвО. 1) Функции $x_{1}, \ldots, x_{n}$, удовлетворяющие уравнению (5) в малой окрестности $U$, задают локальное обрашение отображения Виета, т.е. $V(x(a))=$ $a$, где $x(a)=\left(x_{1}(a), \ldots, x_{n}(a)\right)$. Обозначим через $U_{0}$ образ области $U$ при этом локальном обращении. Рассмотрим пару функциональных полей $V^{*} K_{U} \subset V^{*} P_{U}$ на области $U_{0}$, индуцированных при отображении $V$ из функциональных полей $K_{U} \subset P_{U}$ на области $U$. Поле $V^{*} P_{U}$ изоморфно полю $R\left[x_{1}, \ldots, x_{n}\right]$ всех рациональных функций от $x_{1}, \ldots, x_{n}$. Поле $V^{*} K_{U}$ изоморфно полю $R_{S}\left[x_{1}, \ldots, x_{n}\right]$ всех симметричных рациональных функций от $x_{1}, \ldots, x_{n}$. Действительно, согласно теореме о симметричных рациональных функциях, каждая такая функция является рациональной функцией от $x_{1}+\cdots+x_{n}, \ldots, x_{1} \cdots x_{n}$.

2) Группа $S(n)$ перестановок координат действует без ядра на поле рациональных функций, причем полем инвариантов является поле симметричных рациональных функций. Согласно основной теореме теории Галуа (см. п. 3.2), группа Галуа расширения Галуа $R_{S}\left[x_{1}, \ldots, x_{n}\right] \subset R\left[x_{1}, \ldots, x_{n}\right]$ изоморфна группе $S(n)$.

ТеОрема 3.12 (Абель). Общее алгебраическое уравнение степени $\geqslant 5$ не решается в радикалах.

ДокАЗАТЕльСТво. Группа $S(n)$ при $n \geqslant 5$ неразрешима.

ЗАмечАниЕ. Эта теорема была доказана Абелем еще до возникновения теории Галуа и теории групп. Его оригинальное доказательство ближе к методу Лиувилля, чем к теории Галуа.

3.4. Понижение степени уравнения. Можно ли выразить корни данного алгебраического уравнения степени $n$ через его коэффициенты, используя арифметические операции, присоединение радикалов и присоединение решений и алгебраических уравнений степени $k$ меньше, чем $n$ ? В п. 3.4.2 мы даем ответ на этот вопрос в терминах группы Галуа уравнения. Для этого нам понадобятся свойства подгрупп групшы перестановок. 
3.4.1. Подгрупш групшы перестановок $k$ элементов. Нам понадобится следующая лемма.

Лемма 3.13 [21]. Группа Г изоморфна подгруппе $S(k)$, если и только если группа Г обладает набором подгрупп $\Gamma_{i}, i=1, \ldots, m$, таким, ито

1) группа $\bigcap_{i=1}^{m} \Gamma_{i}$ не содержит нормальных делителей группь $\Gamma$, отличных от тривиального;

2) $\sum_{i=1}^{m} \operatorname{ind}\left(\Gamma, \Gamma_{i}\right) \leqslant k$.

ДокАЗАТЕЛЬСтвО. Пусть Г является подгруппй $S(k)$. Рассмотрим представление группы $\Gamma$ как некоторой подгруппы перестановок множества $M$, содержащего $k$ элементов. Пусть под действием групшы $\Gamma$ множество $M$ распадается на $m$ орбит. Выберем в каждой орбите по точке $x_{i}$. Набор стационарных подгрупп $\Gamma_{i}$ точек $x_{i}$ удовлетворяет условиям леммы.

Обратно, пусть группа Г обладает набором подгрупп, удовлетворяющим условиям леммы. Обозначим через $P$ непересекающееся объединение множеств $P_{i}=\left\{P_{i}^{j}\right\}-$ множеств правых классов смежности $P_{i}^{j}$ группы $\Gamma$ по подгрупе $\Gamma_{i}$. Група $\Gamma$ обладает естественным действием на множестве $P$. Возникающее представление групшы $\Gamma$ в группу $S(P)$ точное, так как ядро этого представления лежит в групе $\bigcap_{i=1}^{m} \Gamma_{i}$. Группа $S(P)$ вкладьвается в групшу $S(k)$, так как в множестве $P$ содержится $\sum_{i=1}^{m} \operatorname{ind}\left(\Gamma, \Gamma_{i}\right) \leqslant k$ элементов.

СлЕДСТВИЕ 3.14. Фактор-группа подгруппь симметрической группь $S(k)$ изоморфна подәруппе симметрической группь $S(k)$.

ДокАЗАТЕЛЬство. Пусть группа $G$ изоморфна подгруппе группы $S(k)$ и $\Gamma_{i}-$ набор ее подгруп, удовлетворяющих условию леммы 3.13. Пусть $\pi$ - произвольный гомоморфизм группы $G$. Тогда совокупность подгрупп $\pi\left(\Gamma_{i}\right)$ в групе $\pi(G)$ тоже удовлетворяет условиям леммы.

ОПРЕДЕЛЕНИЕ. Скажем, что нормальный делитель $H$ в группе $G$ имеет глубину, не превосходящую $k$, если в групе $G$ сушествует подгруппа $G_{0}$ индекса, не превосходящего $k$, такая, что нормальный делитель $H$ является пересечением всех подгруп, сопряженных с подгруппой $G_{0}$. Будем говорить, что группа $G$ имеет глубину, не превосходящую $k$, если единичньй элемент этой группы является ее нормальным делителем глубины, не превосходяшей $k$.

ОПРЕДЕЛЕНИЕ. Нормальной башней групы $G$ называется вложенная цепочка подгруп $G=G_{0} \supset \cdots \supset G_{n}=e$, в которой групша $G_{0}$ совпадает с исходной группй $G$, группа $G_{n}$ тривиальна и для каждого $i=0,1, \ldots, n-1$ группа $G_{i+1}$ является нормальным делителем группы $G_{i}$.

СЛЕДСТВИЕ 3.15. Если группа $G$ является подгруппой групnы $S(k)$, то у групnы $G$ существует нормальная башня $G=G_{0} \supset \cdots \supset G_{n}=e$, в которой для кажддого $i=1, \ldots, n$ группа $G_{i}$ имеет в группе $G_{i-1}$ глубину, не превосходящую $k$.

ДоКАЗАТЕльство. Пусть $\Gamma_{i}$ - совокупность подгрупп в группе $G$, удовлетворяюших условиям леммы 3.13 . Обозначим через $F_{i}$ нормальньй делитель групшы $G$, равньй пересечению всех подгрупп, сопряженных группе $\Gamma_{i}$. Цепочка подгрупп $G_{0}=F_{0}$, $G_{1}=F_{0} \cap F_{1}, \ldots, G_{m}=F_{0} \cap \cdots \cap F_{m}$ удовлетворяет требованиям следствия. 
3.4.2. Условие понижаемости степени уравнения при помощи радикалов. Начнем с формальных определений.

ОПРЕДЕЛЕНИЕ. Алгебраическое уравнение над полем $K$ решается при помощи радикалов и корней уравнений степени не выше $k$ (или, короче, решается в $k$-радикалаx), если сушествует цепочка расширений $K=K_{0} \subset \cdots \subset K_{N}$, в которой поле $K_{i}$ получается из поля $K_{i-1}, i=1, \ldots, N$, либо присоединением радикала, либо присоединением всех корней алгебраического уравнения степени не вьше $k$ с коэффициентами в поле $K_{i-1}$, а поле $K_{N}$ содержит все корни исходного алгебраического уравнения.

ОПРЕДЕЛЕНИЕ. Группа $G$ называется $k$-разрешимой, если у нее сушествует нормальная башня

$$
G=H_{0} \supset \cdots \supset H_{N}=e
$$

такая, что для каждого $i, i=1, \ldots, N$, либо глубина нормального делителя $H_{i}$ в группе $H_{i-1}$ не превосходит $k$, либо фактор-группа $H_{i-1} / H_{i}$ коммутативна.

Теорема 3.16. Алгебраическое уравнение решается в $k$-радикалах, если и только если его группа Галуа $G$ k-разрешима.

Справедлива следующая лемма.

Лемма 3.17. Пусть на поле $Р$ действует конечная группа автоморфизмов $с$ полем инвариантов $P_{0}$. Пусть орбита точки $x \in P$ содержит ровно $m$ әлементов. Тогда $x$ удовлетворяет некоторому алгебраическому уравнению степени т с коэффичиентами из поля $P_{0}$.

ДОКАЗАТЕЛЬСТВО. Пусть $x_{1}, \ldots, x_{m}$ - точки орбиты. Основные симметрические функции $\sigma_{1}, \ldots, \sigma_{m}$ от этих точек остаются неподвижными при действии групшы и, следовательно, принадлежат полю инвариантов. Точки орбиты являются корнями алгебраического уравнения степени $m$

$$
x^{m}-\sigma_{1} x^{m-1}+\cdots+(-1)^{m} \sigma_{m}=0
$$

с коэффициентами из поля инвариантов $P_{0}$.

ЛЕмма 3.18. Пусть на поле $P$ действует конечная группа автоморфизмов $G$ с полем инвариантов $P_{0}$. Пусть глубина группь $G$ не превосходит $k$. Тогда поле $P$ мохсно получить из поля $P_{0}$ присоединением всех корней некоторого алгебраического уравнения степени, не превосходящей $k$, с коэффищиентами из поля $P_{0}$.

ДокАЗАТЕльство. Пусть $G_{0}$ - подгруппа групшы $G$ индекса $m$, не превосходящего $k$, такая, что пересечение всех подгрупп, сопряженных с $G_{0}$, содержит лишш единичньй элемент группы $G$. Согласно основной теореме теории Галуа, подгруппе $G_{0}$ соответствует некоторое промежуточное поле $P_{1} ; P_{0} \subseteq P_{1} \subseteq P$. Поле $P_{1}$, как и любое другое конечное алгебраическое расширение поля $P_{0}$, порождается над полем $P_{0}$ некоторым элементом $x$. Точка $x$ обладает следующим свойством: элемент $g$ группы $G$ оставляет точку $x$ на месте, если и только если $g \in G_{0}$. Орбита точки $x$ относительно 
действия группы $G$ содержит ровно $m$ точек, поскольку индекс стационарной подгруппы точки $x$ равен $m$. Неединичный элемент групшы $G$ задает отличную от тождественной перестановку точек орбиты, поскольку пересечение подгрупп, сопряженных с групой $G_{0}$, совпадает с единичным элементом групшы $G$. Поле, порожденное над $P_{0}$ элементами орбиты, совпадает с полем $P$. Действительно, это поле соответствует тривиальной подгруппе группы Галуа. Лемма 3.18 теперь вытекает из леммы 3.17 .

Вернемся к доказательству теоремы 3.16.

ДОКАЗАТЕЛЬСТВО ТЕОРЕМЫ 3.16. 1) Необходимость условия на групу Галуа доказывается так же, как необходимость разрешимости группы Галуа для разрешимости уравнения в радикалах. Нужно лиш рассмотреть, что происходит с группой Галуа $G_{i}$ нашего уравнения над полем $K_{i}$ при переходе к полю $K_{i+1}$, если поле $K_{i+1}$ получено из поля $K_{i}$ присоединением всех корней уравнения степени $\leqslant k$. В этом случае группа Галуа поля $K_{i+1}$ над полем $K_{i}$ является подгруппой группы $S(k)$ (см. лемму 3.2). Согласно теореме 3.4 о поведении группы Галуа при изменении основного поля, фактор-группа $G_{i} / G_{i+1}$ является фактор-группой некоторой подгрупшы в $S(k)$. Согласно следствиям 3.14 и 3.15, у группы $G_{i} / G_{i+1}$ существует нормальная башня $G_{i} / G_{i+1}=\Gamma_{0} \supset \cdots \supset \Gamma_{m}=e$ такая, что группа $\Gamma_{i+1}$ имеет в группе $\Gamma_{i}$ глубину $\leqslant k$. Между группами $G_{i} \supset G_{i+1}$ достаточно вставить цепочку подгрупп

$$
G_{i}=\Gamma_{0 i} \supset \cdots \supset \Gamma_{m i}=G_{i+1}
$$

где $\Gamma_{p i}-$ прообраз группы $\Gamma_{p}$ при гомоморфизме факторизации $G_{i} \rightarrow G_{i} / G_{i+1}$. Ясно, что группа $\Gamma_{p+1, i}$ является нормальным делителем глубины $\leqslant k$ в группе $\Gamma_{p i}$. Это и доставляет недостающее звено в доказательстве необходимости условия на групу Галуа.

2) Достаточность условия на групу Галуа доказьвается индукшией по длине $N$ нормальной башни в группе $G$. Группа $G$ имеет $k$-разрешимьй нормальный делитель $H_{1}$ с нормальной башней длины $N-1$. Обозначим через $P_{1}$ поле инвариантов групшы $H_{1}$. Согласно индукционному предположению, поле $P$ получается из поля $P_{1}$ при помоши присоединений радикалов и корней уравнений степени не выше $k$. Фактор-группа $G / H_{1}$ действует на поле $P_{1}$ с полем инвариантов $P_{0}$. Если фактор-группа $G / H_{1}$ коммутативна, то поле $P_{1}$ получается из поля $P_{0}$ при помоши присоединения радикалов (см. утверждение 3.7 ). Если фактор-группа $G / H_{1}$ имеет глубину, не превосходящую $k$, то поле $P_{1}$ получается из поля $P$ присоединением всех корней алгебраического уравнения степени не выше $k$ (см. лемму 3.18 ).

Теорема 3.19. Общее алгебраическое уравнение (см. п. 3.3.3) степени $n \geqslant 5$ не решается при помощи радикалов и корней алгебраических уравнений степени меньше $n$.

ДокАЗАТЕльство. Группа Галуа общего алгебраического уравнения степени $n$ изоморфна групше $S(n)$ (см. п. 3.3.3).

При $n \geqslant 5$ группа $S(n)$ имеет всего лишш две различных башни нормальных подгрупп: тривиальную башню $e \subset S(n)$ и башню $e \subset A(n) \subset S(n)$, где $e$ - единичная подгруппа, а $A(n)$ - знакопеременная подгруппа. Поэтому група $S(n)$ при $n \geqslant 5$ не является разрешимой. 


\section{$\S$ 4. Разрешимость линейных дифференциальных уравнений в квадратурах и теория Пикара-Вессио}

Пикар обратил внимание на аналогию между линейными дифференциальными уравнениями и алгебраическими уравнениями и начал строить дифференшиальный аналог теории Галуа. Венцом этой теории является теорема Пикара-Вессио, в которой вопрос о разрешимости или неразрешимости линейного дифференциального уравнения связьвается с вопросом о разрешимости или неразрешимости некоторой алгебраической группы Ли.

4.1. Аналогия между линейными дифференциальными уравнениями и алгебраическими уравнениями. Напомним простейшие свойства линейных дифференциальных уравнений и их аналоги для алгебраических уравнений.

4.1.1. Деление с остатком и наибольший обший делитель дифференшиальных операторов. Линейным дифференииальным оператором порядка $n$ над дифференциальньм полем $K$ называется оператор $L=a_{n} D^{n}+\cdots+a_{0}$, где $a_{i} \in K$ и $a_{n} \neq 0$, действующий на элементы $y$ поля $K$ по формуле

$$
L(y)=a_{n} y^{(n)}+\cdots+a_{0} y
$$

Для операторов $L_{1}$ и $L_{2}$ над $K$ их произведение $L=L_{1} \circ L_{2}=L_{1}\left(L_{2}\right)$ тоже является оператором над $K$. Произведение операторов, вообще говоря, некоммутативно, но в старшем члене эта некоммутативность не проявляется. Именно, стариий член $a_{n} D^{n}$ оператора $L$ для $L=L_{1} \circ L_{2}$ равен $b_{m} c_{k} D^{m+k}$, где $b_{m} D^{m}$ u $c_{k} D^{k}$ - cmaриие члены операторов $L_{1}$ u $L_{2}$.

Для операторов $L$ и $L_{2}$ порядков $n$ и $k$ над $K$ существуют и единственны операторы $L_{1}$ и $R$ над $K$ такие, что $L=L_{1} \circ L_{2}+R$ и порядок оператора $R$ строго меньше, чем $k$. Оператор $R$ называется остатком от деления справа оператора $L$ на оператор $L_{2}$. Операторы $L_{1}$ и $R$ строятся по операторам $L$ и $L_{2}$ явно: алгоритм деления операторов с остатком основан на приведенной вьше формуле для старшего члена произведения операторов и абсолютно аналогичен алгоритму деления с остатком полиномов от одной переменной.

Для любых двух операторов $L_{1}$ и $L_{2}$ над $K$ можно явно найти их правьй наибольший общий делитель $N$, т.е. оператор $N$ над $K$ наибольшего возможного порядка, которьй делит справа операторы $L_{1}$ и $L_{2}$, т.е. $L_{1}=M_{1} \circ N$ и $L_{2}=M_{2} \circ N$, где $M_{1}$ и $M_{2}$ - некоторые операторы над $K$. Нахождение операторов $M_{1}, M_{2}$ и $N$ по операторам $L_{1}$ и $L_{2}$ абсолютно аналогично алгоритму Евклида нахождения наибольшего общего делителя двух полиномов от одной переменной и основано на алгоритме деления операторов с остатком. Как и в коммутативном случае, наибольший обший делитель $N$ представим в виде $N=A L_{1}+B L_{2}$, где $A, B$ - некоторые операторынад $K$.

Ясно, что $y$ является решением уравнения $N(y)=0$, если и только если $L_{1}(y)=0$ и $L_{2}(y)=0$.

4.1.2. Понижение порядка линейного дифференщиального уравнения как аналог теоремы Безу. Пусть $L$ - линейньй дифференциальньй оператор над $K, y_{1}$ - ненулевой элемент поля $K, p=\frac{y_{1}^{\prime}}{y_{1}}$ - его логарифмическая производная и $L_{2}=D-p$-оператор первого порядка, аннулируюший $y_{1}$. Остаток $R$ от деления справа $L$ на $L_{2}$ является 
оператором умножения на $c_{0}$, где $c_{0}=\frac{1}{y_{1}} L\left(y_{1}\right)$. Действительно, нужное равенство получается при подстановке $y=y_{1}$ в тождество $L(y) \equiv L_{1} \circ L_{2}(y)+c_{0} y$. Оператор $L$ делится справа на оператор $L_{2}$, если и только если элемент $y_{1}$ удовлетворяет тождеству $L\left(y_{1}\right) \equiv 0$.

Используя ненулевое решение $y_{1}$ уравнения $n$-го порядка $L(y)=0$, можно понизить порядок этого уравнения. Для этого надо представить оператор $L$ в виде $L=L_{1} \circ L_{2}$, где $L_{1}$-оператор $(n-1)$-го порядка. Коэффиииенты оператора $L_{1}$ лежат в расширении дифференциального поля $K$ логарифмической производной р әлемента $y_{1}$. Если известно какое-либо решение $u$ уравнения $L_{1}(u)=0$, то по нему можно построить некоторое решение $y$ исходного уравнения $L(y)=0$. Для этого достаточно решить уравнение $L_{2}(y)=y^{\prime}-p y=u$. Описанная процедура называется понижением порядка дифференциального уравнения.

ЗАмечАниЕ. Оператор, аннулирующий $y_{1}$, определен с точностью до умножения на произвольную функцию, и от выбора этой функции зависит процедура понижения порядка. Легче делить на оператор $\widetilde{L}_{2}=D \circ y_{1}^{-1}$, являюшийся композицией умножения на элемент $y_{1}^{-1}$ и дифференцирования. Для этого достаточно вычислить оператор $L_{3}=L \circ y_{1}$, являюшийся композицией умножения на элемент $y_{1}$ и оператора $L$. Оператор $L_{3}$ делится справа на $D$, т.е. $L_{3}=\widetilde{L}_{1} \circ D$, так как $L_{3}(1) \equiv L \circ y_{1}(1) \equiv 0$. Видно, что $L=\widetilde{L}_{1} \circ \widetilde{L}_{2}$. Исходное уравнение $L(y)=0$ сводится к уравнению $\widetilde{L}_{1}(u)=0$ меньшего порядка. Обычно именно такую процедуру понижения порядка приводят в учебниках по дифференциальным уравнениям. Отметим, что коэффициенты оператора $\widetilde{L}_{1}$ лежат в расширении дифференциального поля $K$ самим элементом $y_{1}$, а не его логарифмической производной $p$, что иногда делает оператор $\widetilde{L}_{1}$ менее удобным, чем оператор $L_{1}$.

В алгебре есть следующие аналоги приведенных фактов: 1) остаток от деления полинома $P$ переменной $x$ на $(x-a)$ равен значению полинома $P$ в точке $a$ (теорема Безу); 2) если известно одно решение $x_{1}$ уравнения $P(x)=0$, то его степень можно понизить: остальные корни полинома $P$ удовлетворяют уравнению меньшей степени $Q(x)=0$, где $Q=P:\left(x-x_{1}\right)$. Кроме аналогии здесь имеется и отличие: решения дифференщиального уравнения, полученного процедурой понижения порядка, вообше говоря, не являются решениями исходного уравнения.

ЗАмЕчАнИЕ. Экспоненты являются собственньми функциями дифференциальных операторов $P(D)$ с постоянными коэффициентами. Этот факт эквивалентен теореме Безу. Действительно, если $P=Q(x-a)+P(a)$, то $P(D)=Q(D) \circ(D-a)+P(a)$. Поэтому решение $y_{1}$ дифференциального уравнения $(D-a) y=0$ является собственным вектором оператора $P(D)$ с собственным числом $P(a)$.

4.1.3. Аналог формул Виета для дифференшиальных операторов. Если известны все корни $x_{1}, \ldots, x_{n}$ полинома $P$ степени $n$ со старшим коэффищиентом 1 , то полином $P$ можно восстановить: по формулам Виета $P(x)=x^{n}+p_{1} x^{n-1}+\cdots+p_{n}$, где $p_{1}=-\sigma_{1}$, $\ldots, p_{n}=(-1)^{n} \sigma_{n}$ и $\sigma_{1}=x_{1}+\cdots+x_{n}, \ldots, \sigma_{n}=x_{1} \cdots x_{n}$. Функции $\sigma_{1}, \ldots, \sigma_{n}$ не меняются при перестановке корней и называются основньми симметрическими функциями.

Аналогично этому, если известны $n$ линейно независимых решений $y_{1}, \ldots, y_{n}$ линейного дифференциального уравнения $n$-го порядка $L=0$, где $L$ - оператор, у ко- 
торого коэффициент при старшей производной равен единице, то оператор $L$ можно восстановить. Действительно, прежде всего такой оператор не более чем один: разность $L_{1}-L_{2}$ двух операторов, обладающих этими свойствами, является оператором порядка $<n$, имеюшим $n$ линейно независимых решений, что возможно, лишш если $L_{1}$ совпадает с $L_{2}$.

Вронскиан $W$ от $n$ независимых решений $y_{1}, \ldots, y_{n}$ линейного дифференциального уравнения не равен нулю. Рассмотрим уравнение $W\left(y, y_{1}, \ldots, y_{n}\right)=0$, где $W\left(y, y_{1}, \ldots, y_{n}\right)$ - вронскиан от неизвестной функщии $y$ и функций $y_{1}, \ldots, y_{n}$. Раскрывая вронскиан

$$
W\left(y, y_{1}, \ldots, y_{n}\right)=\left|\begin{array}{cccc}
y & y_{1} & \ldots & y_{n} \\
\vdots & \vdots & & \vdots \\
y^{(n)} & y_{1}^{(n)} & \ldots & y_{n}^{(n)}
\end{array}\right|
$$

по первому столбцу и деля его на $W$, получим уравнение

$$
y^{(n)}+p_{1} y^{(n-1)}+\cdots+p_{n} y=0,
$$

в котором $p_{1}=-\varphi_{1}, \ldots, p_{n}=(-1)^{n} \varphi_{n}$, где

$$
\varphi_{1}=\frac{\left|\begin{array}{ccc}
y_{1} & \ldots & y_{n} \\
\vdots & & \vdots \\
y_{1}^{(n-2)} & \ldots & y_{n}^{(n-2)} \\
y_{1}^{(n)} & \ldots & y_{n}^{(n)}
\end{array}\right|}{W}, \ldots, \quad \varphi_{n}=\frac{\left|\begin{array}{ccc}
y_{1}^{\prime} & \ldots & y_{n}^{\prime} \\
\vdots & & \vdots \\
y_{1}^{(n-1)} & \ldots & y_{n}^{(n-1)} \\
y_{1}^{(n)} & \ldots & y_{n}^{(n)}
\end{array}\right|}{W} .
$$

Функции $y_{1}, \ldots, y_{n}$ и их линейные комбинации является решениями уравнения (6). Формулы (6), (7) вполне аналогичны формулам Виета.

Функции $\varphi_{1}, \ldots, \varphi_{n}$ являются рациональными функциями от функций $y_{1}, \ldots, y_{n}$ и от их производных до порядка $n$. Эти функции зависят лишь от линейного пространства $V$, натянутого на функщии $y_{1}, \ldots, y_{n}$, но не зависят от выбора конкретного базиса $y_{1}, \ldots, y_{n}$ в пространстве $V$. Другими словами, функции $\varphi_{1}, \ldots, \varphi_{n}$ являются $G L(V)$ инвариантньми функциями от $y_{1}, \ldots, y_{n}$ и от их производных. Функции $\varphi_{1}, \ldots, \varphi_{n}$ будем назьвать основными дифференииальными инвариантами от $y_{1}, \ldots, y_{n}$.

4.1.4. Аналог теоремы о симметричных функциях для дифференшиальных операторов. Как известно из алгебры, всякая рациональная функция от переменных $x_{1}, \ldots, x_{n}$, не меняюшаяся при перестановках переменных, на самом деле является рациональной функцией основных симметрических функций $\sigma_{1}, \ldots, \sigma_{n}$ переменных $x_{1}, \ldots, x_{n}$. Другими словами, всякое рациональное выражение, симметрично зависяшее от корней полинома степени $n$, рационально выражается через коэффициенты этого полинома.

Аналогичная теорема для линейных дифференциальных уравнений была открыта Пикаром.

ТеОрема 4.1. Всякая рациональная функиия $R$ от линейно независимых функций $y_{1}, \ldots, y_{n}$ и их производных, которая является $G L(V)$-инвариантной (т.е. которая не изменится, если функции $y_{1}, \ldots, y_{n}$ заменить их линейньми комбинациями $z_{1}=a_{11} y_{1}+\cdots+a_{1 n} y_{n}, \ldots, z_{n}=a_{n 1} y_{1}+\cdots+a_{n n} y_{n}$ при условии, что матрица $A=\left\{a_{i j}\right\}$ невырождена), на самом деле является рациональной функцией 
от основных дифференииальных инвариантов $\varphi_{1}, \ldots, \varphi_{n}$ функций $y_{1}, \ldots, y_{n}$ и от производных этих инвариантов.

ДокАЗАТЕЛЬСТвО. Каждая функция $y$ в пространстве $V$, натянутом на $y_{1}, \ldots, y_{n}$, удовлетворяет тождеству $y^{(n)}-\varphi_{1} y^{(n-1)}+\cdots+(-1)^{n} \varphi_{n} y=0$. Дифференцируя это тождество, можно выразить любую производную функции $y$ порядка $\geqslant n$ через функцию $y$, ее производные порядка $<n$, основные дифференциальные инварианты и их производные. Подставляя найденные выражения старших производных функций $y_{1}, \ldots, y_{n}$ в рациональную функцию $R$, мы получим рациональную функцию $\widetilde{R}$ от функций $\varphi_{1}, \ldots, \varphi_{n}$, их производных и от элементов фундаментальной матрицы $Y$, где

$$
Y=\left|\begin{array}{ccc}
y_{1} & \ldots & y_{n} \\
\vdots & & \vdots \\
y_{1}^{(n-1)} & \ldots & y_{n}^{(n-1)}
\end{array}\right| .
$$

Функция $\widetilde{R}$ не может меняться при линейных преобразованиях пространства $V$, натянутого на $y_{1}, \ldots, y_{n}$. Любая невырожденная $(n \times n)$-матрица может быть получена как образ фундаментальной матрицы $Y$ при некотором линейном преобразовании пространства $V$. Рациональная функция $\widetilde{R}$ должна быть постоянна на множестве невырожденных матрищ, поэтому она постоянна на множестве всех матрищ, не зависит от матрищы $Y$, а зависит лишь от дифференциальных инвариантов и их производных.

СлЕДСТВИЕ 4.2. Всякая рациональная функиия от независимых решений $y_{1}, \ldots, y_{n}$ линейного дифференциального уравнения $u$ от их производных, которая не меняется при выборе другого базиса $z_{1}, \ldots, z_{n}$ в пространстве решений, является рациональной функиией от коэффициентов дифференциального уравнения и от их производных.

4.2. Группа Галуа линейного дифференциального уравнения. Рассмотрим линейное дифференциальное уравнение

$$
y^{(n)}+p_{1} y^{(n-1)}+\cdots+p_{n} y=0
$$

с коэффициентами в некотором функциональном дифференциальном поле $K$. (Напомним, что мы всегда предполагаем, что поле $K$ содержит все комплексные константы.)

Дифференциальным полиномом над $K$ от функций $u_{1}, \ldots, u_{n}$ называется полином с коэффициентами из $K$ от функции $u_{1}, \ldots, u_{n}$ и от их производных. Дифференциальным соотношением над $K$ между решениями $y_{1}, \ldots, y_{n}$ уравнения (8) назьвается дифференциальный полином над полем $K$ от функций $u_{1}, \ldots, u_{n}$, которьй обрашается в нуль при подстановке $u_{1}=y_{1}, \ldots, u_{n}=y_{n}$.

ОПРЕДЕЛЕНИЕ. Группой Галуа дифференциального уравнения (8) над дифференииальным полем $K$ назьвается подгруппа $G$ группы $G L(V)$ всех линейных преобразований пространства решений $V$ уравнения (8), сохраняюших все дифференциальные соотношения над $K$ между решениями уравнения (т.е. если $A \in G$ и $Q$ - любое соотношение над $K$ между некоторыми решениями $y_{1}, \ldots, y_{n}$, то решения $A y_{1}, \ldots, A y_{n}$ должны быть связаны тем же соотношением $Q$ ). 
УТВЕРЖДЕНИЕ 4.3. Группа Галуа линейного дифференииального уравнения является алгебраической подгруппой в $G L(V)$.

ДоКАЗАТЕЛЬСТВо. Очевидно, что для каждого дифференциального соотношения $Q$ между решениями $y_{1}, \ldots, y_{n}$ множество линейных преобразований $A$, для которых соотношение $Q$ выполняется для $A y_{1}, \ldots, A y_{n}$, является алгебраическим. Пересечение любого числа алгебраических многообразий является алгебраическим многообразием.

ОПРЕДЕЛЕНИЕ. Функциональное дифференциальное поле $P$ назьвается расширением Пикара-Вессио функционального дифференциального поля $K$, если сушествует линейное дифференциальное уравнение (8) с коэффициентами в дифференциальном поле $K$ такое, что $P$ получается присоединением к $K$ всех решений уравнения (8). Груnпой Галуа расширения Пикара-Вессио над полем $K$ называется группа всех автоморфизмов дифференциального поля $P$, оставляюших на месте все элементы поля $K$.

Каждый элемент $\tau$ из групш Галуа дифференциального поля $P$ над дифференциальньм полем $K$ задает линейное преобразование пространства решений и сохраняет все дифференциальные соотношения, определенные над полем $K$ между решениями. Таким образом, група Галуа дифференциального поля $P$ над $K$ имеет представление в групше Галуа $G$ уравнения (8), определяющее расширение Пикара-Вессио $P$. Очевидно, что это представление является изоморфизмом груп, т.е. группа Галуа уравнения и групша Галуа заданного им расширения Пикара-Вессио изоморфны. Используя этот изоморфизм, можно определить на группе Галуа расширения Пикара-Вессио структуру алгебраической группы. Если два различных линейных дифференциальных уравнения над полем $K$ задают одно и то же расширение Пикара-Вессио, то группы Галуа этих уравнений изоморфны не только как абстрактные групшы, но и как алгебраические групшы. Поэтому структура алгебраической группы на группе расширения Пикара-Вессио определена корректно.

4.3. Основная теорема теории Пикара-Вессио. Пусть $P$ - расширение Пикара-Вессио дифференциального поля $K$ и $G$ - его группа Галуа.

Определены следующие отображения между множеством промежуточных дифференциальных полей $F, K \subseteq F \subseteq P$, и множеством подгрупп группы Галуа $G$.

1) Oтображсение $F d$, сопоставляющее каждой подгруппе Г группы $G$ дифференциальное поле $F d(\Gamma)$, состоящее из элементов поля $P$, остаюшихся неподвижными при действии подгрупшы $\Gamma$ (ясно, что $K \subseteq F d(\Gamma))$.

2) Отображение $G p$, сопоставляющее каждому промежуточному дифференциальному полю $F, K \subseteq F \subseteq P$, подгрупу $G p(F) \subseteq G$, являюшуюся группой Галуа расширения Пикара-Вессио $P$ поля $F$ ( $P$ является расширением Пикара-Вессио поля $K$, и поэтому оно автоматически является расширением Пикара-Вессио промежуточного поля $F, K \subset F \subset P)$.

Отображения $F d$ и $G p$ устанавливают соответствие Галуа между подгруппами групшы Галуа и промежуточными дифференциальными полями расширения Пикара-Вессио. Приведем без доказательства следующую теорему.

ОСНОВНАЯ ТеОРема 4.4 (теории Пикара-Вессио). Для всякого расширения $P$ Пикара-Вессио дифференииального поля $K$ с әруппой Галуа $G$ 
1) композиция отображений $F d$ и Gр является тождественным отображением множества промежуточных полей в себя: если $F$ - дифференциальное поле и $K \subseteq F \subseteq P$, mо $F d(G p(F))=F$;

2) композиция отображений $G p$ и $F d$ сопоставляет каждой подгруппе $\Gamma$ в группе Галуа $G$ ее алгебраическое замыкание $\bar{\Gamma}$ в группе $G$ : если $\Gamma$ - подгруппа группы Галуа, $\Gamma \subset G, \operatorname{mo~} G p(F d(\Gamma))=\bar{\Gamma}$;

3) промехуточное дифференциальное поле $F, K \subseteq F \subseteq P$, является расширением Пикара-Вессио поля $K$, если и только если группа $G p(F)$ является нормальным делителем группь $G$. При этом группа Галуа расширения ПикараВессио $F$ поля $K$ является фактор-группой группь $G$ по нормальному делителю $G p(F)$.

Докажем полезное характеристическое свойство расширений Пикара-Вессио, непосредственно вытекающее из основной теоремы.

СледСтвиЕ 4.5. Дифференциальное поле $P$ является расширением ПикараВессио дифференциального поля $K, K \subseteq P$, если и только если существует группа Г автоморфизмов дифференииального поля $P$ такая, что 1) она оставляет неподвижныци все әлементы поля $K$ и только их; 2) существует лежащее в $P$ конечномерное линейное пространство $V$ над полем констант, инвариантное относительно группь $\Gamma$ и такое, что поле $Р$ является наименьшим дифференциальным м полем, содержащим $V$ и K.

ДокАЗАтельство. Расширения Пикара-Вессио указанными свойствами обладают. Это вытекает из п. 1) основной теоремы, примененной к полю $F=K$. Обратно, пусть $y_{1}, \ldots, y_{n}$ - базис линейного пространства $V$, о котором идет речь в п. 2) следствия. Коэффициенты линейного дифференциального уравнения $n$-го порядка, которому удовлетворяют функции $y_{1}, \ldots, y_{n}$, инвариантны относительно всех линейных преобразований пространства $V$. Поэтому они инвариантны относительно групшы $\Gamma$ и лежат в $K$. Следовательно, $P$ получается из $K$ присоединением всех решений указанного уравнения и $P$ является расширением Пикара-Вессио поля $K$.

Что произойдет с группой Галуа линейного дифференциального уравнения, если дифференщиальное поле коэффищиентов $K$ расширить, заменив его бо́льшим дифференциальным полем $K_{1}$ ? Этот вопрос особенно интересен в том случае, когда поле $K_{1}$ является расширением Пикара-Вессио поля $K$. Обозначим через $G_{1}$ группу Галуа расширения $K_{1}$ дифференциального поля $K$. Результаты о неразрешимости линейных дифференциальных уравнений основаны на следующей теореме из теории Пикаpa-Вессио, которую мы приводим без доказательства и которая формулируется вполне аналогично теореме 3.4 .

TеОРема 4.6 (об изменении группы Галуа уравнения при расширении Пикара-Вессио поля коэффициентов). При замене дифференииального поля коэффиuиентов $K$ его расширением Пикара-Вессио $K_{1}$ группа Галуа $G$ уравнения заменяется некоторым своим алгебрачческим нормальным делителем Н. Фактор-группа $G / H$ группь $G$ относительно этого нормального делителя изоморфна некоторой алгебраической фактор-группе группь Галуа $G_{1}$ нового дифференциального поля $K_{1}$ над старыцм дифференциальныц полем $K$. 
4.4. Простейшие расширения Пикара-Вессио. В этом пункте рассматривается следуюшие простейшие расширения Пикара-Вессио: алгебраическое расширение, присоединение интеграла и присоединение экспоненты интеграла.

4.4.1. Алгебраическое расширение. Рассмотрим алгебраическое уравнение

$$
Q(x)=x^{n}+a_{n-1} x^{n-1}+\cdots+a_{0}=0
$$

над функциональным дифференциальньм полем $K$ и расширение Галуа $P$, получающееся присоединением к полю $K$ всех решений уравнения (9).

ЛЕмма 4.7. Поле $Р$ является дифференциальным полем. Каждый автоморфизм поля $P$ над полем $K$, который сохраняет лишь арифметические операции в поле $P$, автоматически сохраняет и операчию дифференцирования.

ДокАЗАТЕЛЬСтво. Изменив, если нужно, алгебраическое уравнение (9), можно считать, что оно неприводимо над полем $K$ и что каждый корень $x_{i}$ уравнения (9) порождает поле $P$ над полем $K$. Дифференцируя тождество $Q\left(x_{i}\right)=0$, получим $\frac{\partial Q}{\partial x}\left(x_{i}\right) x_{i}^{\prime}+\frac{\partial Q}{\partial t}\left(x_{i}\right)=0$, где $\frac{\partial Q}{\partial t}=\sum_{i=1}^{n-1} a_{i}^{\prime} x^{i}$. Полином $\frac{\partial Q}{\partial t}$ не может обрашаться в нуль в точке $x_{i}$, так как уравнение $Q=0$ неприводимо. Получаем алгебраическое выражение производной $x_{i}^{\prime}=-\frac{\partial Q}{\partial x}\left(x_{i}\right) / \frac{\partial Q}{\partial t}\left(x_{i}\right)$ корня $x_{i}$, которое одинаково для всех корней $x_{i}$ полинома $Q$. Отсюда и вытекают оба утверждения леммы.

Группа Галуа $Г$ расширения Галуа $P$ над полем $K$ оставляет неподвижньми лишш элементы поля $K$. Линейное пространство $V$ над полем констант, натянутое на корни $x_{1}, \ldots, x_{n}$ уравнения $(9)$, инвариантно относительно действия групшы $Г$. Согласно следствию 4.5, дифференциальное поле $P$ является расширением Пикара-Вессио. Группа Галуа расширения Пикара-Вессио $P$ поля $K$ совпадает с группой Галуа алгебраического уравнения (9). Основная теорема теории Пикара-Вессио для расширения Пикара-Вессио $P$ дифференииального поля $K$ совпадает с основной теоремой теории Галуа расширения Галуа $P$ поля $K$.

4.4.2. Присоединение интеграла. Пусть $y_{1}$ - интеграл над функциональным дифференщиальным полем $K$ и $y_{1}^{\prime}=a, a \in K, a \neq 0$.

Однородное дифференциальное уравнение $a y^{\prime \prime}-a^{\prime} y^{\prime}=0$ имеет своими независимыми решениями $y_{1}$ и единицу. Расширение дифференциального поля $K$, полученное из $K$ присоединением элемента $y_{1}$, является поэтому расширением Пикара-Вессио (напомним, что мы всегда предполагаем, что поле $K$ содержит все комплексные постоянные).

ЛЕмма 4.8. Интеграл у либо принадлежит полю $K$, либо трансцендентен над полем $K$.

ДокАЗАТЕльство. Допустим, что интеграл $y_{1}$ алгебраичен над полем $K$. Пусть $Q(y)=a_{n} y^{n}+\cdots+a_{0}=0-$ неприводимое над $K$ уравнение, которому удовлетворяет $y_{1}$. Можно считать, что $n>1$ и что $a_{n}=1$. Продифференцировав тождество $Q(y)=0$, получим уравнение меншей степени $n a y^{n-1}+\cdots+a_{0}^{\prime}=0$, которому удовлетворяет $y_{1}$. Это противоречит неприводимости полинома $Q$. 
Пусть элемент $y_{1}$ трансцендентен над $K$. Покажем, что единственное независимое дифференщиальное соотношение над $K$, которому удовлетворяет $y_{1}$, есть $y_{1}^{\prime}=a$. Действительно, используя это соотношение, каждьй дифференциальный полином над $K$ от $y_{1}$ можно переписать как полином от $y_{1}$ с коэффициентами из $K$. Но ни один такой нетривиальный полином не может обратиться в нуль, так как элемент $y_{1}$ трансцендентен над $K$. Поэтому група Галуа уравнения $a y^{\prime \prime}-a^{\prime} y^{\prime}=0$ состоит из линейных преобразований вида $A y_{1}=y_{1}+C, A(1)=1$, где $C$ - любое комплексное число. Итак, группа Галуа нетривиального интегрального расширения изоморфна аддитивной группе комплексных чисел.

В терминологии Колчина [26] алгебраическая группа называется антикомпактной, если она не содержит элементов конечного порядка, отличных от единицы. Очевидно, что группа Галуа нетривиального интегрального расширения антикомпактна.

УТВЕРЖДЕНИЕ 4.9. Не существует дифференииальных полей между полем $K$ $u K\langle y\rangle$, где $y$ - интеграл над $K$, не лежащий в $K$.

ДокАЗАТЕльство. Действительно, пусть $F$ - дифференциальное поле такое, что $K \subset F \subseteq K\langle y\rangle$. Пусть $b \in F$ и $b \notin K$. Тогда элемент $b$ представим в виде нетривиальной рациональной функции от $y$ с коэффициентами из $K$. Существование такой функции означает, что элемент $y$ алгебраичен над $F$. Но элемент $y$ является интегралом над $F$, так как $y^{\prime}=a \in K$. Интеграл алгебраичен над дифференщиальным полем, если и только если он лежит в этом поле (см. лемму 4.8), т.е. $F=K\langle y\rangle$.

Утверждение доказывает основную теорему теории Пикара-Вессио для присоединения интеграла. Действительно, группа Галуа $C$ поля $K\langle y\rangle$ над полем $K$ не имеет алгебраических подгрупп, а пара дифференциальных полей $K \subset K\langle y\rangle$ не содержит промежуточных диффференциальных полей.

4.4.3. Присоединение экспоненты интеграла. Пусть $y_{1}$ - экспонента интеграла над функциональным дифференциальным полем $K$, т.е. $y_{1}^{\prime}=a y_{1}$, где $a \in K$. Расширение поля $K$ элементом $y_{1}$ является по определению расширением Пикара-Вессио.

ЛЕмМа 4.10. Пусть экспонента интеграла $y_{1}$ является алгебраичным над полем $K$ әлементом. Тогда $y_{1}-$ радикал над полем $K$.

ДокАЗАТЕЛЬСТВО. Пусть $Q(y)=a_{n} y^{n}+\cdots+a_{0}=0-$ неприводимое над $K$ уравнение, которому удовлетворяет элемент $y_{1}$. Можно считать, что $n>1$, $a_{n} \neq 0$ и $a_{0}=1$. Продифференцировав тождество $Q\left(y_{1}\right)=0$, получим уравнение $\sum\left(a_{k}^{\prime}+k a_{k} a\right) y^{k}=0$, которому удовлетворяет $y_{1}$. Это уравнение имеет степень $\leqslant n$, но не содержит свободного члена. Все коэффищиенты этого уравнения должны тождественно обратиться в нуль, так как в противном случае мы получим противоречие с неприводимостью полинома $Q$. Равенство $a_{n}^{\prime}+n a_{n} a=0$ означает, что частное $a_{n} / y_{1}^{n}=c$ является константой. Действительно, из соотношения $y_{1}^{\prime}=a y_{1}$ вытекает, что $\left(y_{1}^{-n}\right)^{\prime}+n a\left(y_{1}^{-n}\right)=0$, т.е. что $y_{1}^{-n}$ и $a_{n}$ удовлетворяют одному и тому же уравнению. Поэтому $y^{n}=a_{n} / c$. Лемма доказана.

Допустим, что элемент $y_{1}$ трансцендентен над $K$. Покажем, что в этом случае единственное независимое дифференциальное соотношение над $K$, которому удовлетворяет $y_{1}$, есть $y_{1}^{\prime}=a y_{1}$. Действительно, используя это соотношение, каждьй дифференциальньй полином над $K$ от $y_{1}$ можно переписать как полином от $y_{1}$ с коэффициентами из $K$. Но ни один такой нетривиальньй полином не может обратиться в нуль, 
так как $y_{1}$ трансцендентен над $K$. Поэтому группа Галуа уравнения $y^{\prime}=a y$ состоит из линейных преобразований вида $A y_{1}=C y_{1}$, где $C \neq 0$ - любое ненулевое комплексное число. Итак, группа Галуа неалгебраического расширения, являющегося присоединением әкспоненты интеграла, совпадает с мультипликативной группой $\mathbb{C}^{*}$ ненулевых комплексных чисел.

Экспонента интеграла над $K$ является алгебраическим элементом $y$ над $K$, если и только если $y$ - радикал над $K$. Поэтому если присоединение экспоненты интеграла является алгебраическим расширением, то его группа Галуа является конечной мультипликативной подгруппой в $\mathbb{C}^{*}$.

В терминологии Колчина [26] алгебраическая группа называется квазикомпактной, если каждая ее неединичная подгруппа содержит элементы конечного порядка, отличные от единицы. Очевидно, что группа Галуа неалгебраического расширения, полученного присоединением экспоненты интеграла, квазикомпактна.

УТВЕРЖДЕНИЕ 4.11. Пусть $у$ - әкспонента интеграла над $K$ и әлемент $у$ трансцендентен над $K$. Тогда каждому неотричательному иелому числу $n$ можно поставить в соответствие дифференциальное поле между полями $K$ $u K\langle y\rangle$. Именно, это дифференциальное поле $K_{n}$, состоящее из рациональных функций от элемента $y^{n}$ с коэффициентами из поля $K$. Для разных $n$ поля $K_{n}$ различны. Всякое промежуточное дифференциальное поле совпадает с некоторымм полем $K_{n}$.

ДОКАЗАТЕЛЬСТВО. Пусть $F$ - дифференщиальное поле, строго содержащее поле $K$ и лежащее в поле $K\langle y\rangle$. Повторяя рассуждения утверждения 4.9 , получаем, что элемент $y$ алгебраичен над $F$. Элемент $y$ является экспонентой интеграла над $F$. Поэтому неприводимое над полем $F$ алгебраическое уравнение, которому удовлетворяет $y$, имеет вид $y^{n}-a=0$, где $a \in F$ (см. лемму 4.10), и, значит, $K_{n} \subseteq F$. Поле $K_{n}$ должно совпадать с $F$. Действительно, в противном случае существует элемент $b \in F$, $b \notin K_{n}$. Элемент $b$ является некоторой рациональной функцией $R$ от $y$, и соотношение $R(y)$ не является следствием уравнения $y^{n}=a$. Это противоречит неприводимости уравнения $y^{n}=a$. Противоречие доказывает, что $K_{n}=F$. Поля $K_{n}$ при разных $n$ различны в силу трансцендентности $y$ над $K$.

Утверждение доказывает основную теорему теории Пикара-Вессио для присоединения әкспоненты интеграла. Действительно, каждая собственная алгебраическая подгрупа группы $\mathbb{C}^{*}$ является групой корней $n$-й степени из единицы для некоторого $n$. Промежуточное между $K$ и $K\langle y\rangle$ дифференциальное поле состоит в точности из элементов поля $K\langle y\rangle$, которые остаются на месте при действии групшы корней $n$-й степени из единищы на $K\langle y\rangle$.

4.5. Разрешимость дифференциальных уравнений. Скажем, что алгебраическая группа $G$ является разрешимой, $k$-разрешимой или почти разрешимой в категории алгебраических групп, если у нее сушествует нормальная башня алгебраических подгрупп $G=G_{0} \supset \cdots \supset G_{m}=e$ со следуюшими свойствами

а) для разрешимых групп: для каждого $i=1, \ldots, m$ фактор-группа на $G_{i-1} / G_{i}$ коммутативна (т.е. группа $G$ разрешима);

б) для $k$-разрешимых групп: для каждого $i=1, \ldots, m$ либо глубина группы $G_{i}$ в групе $G_{i-1}$ не превосходит $k$, либо группа $G_{i-1} / G_{i}$ коммутативна; 
в) для почти разрешимых групп: для каждого $i=1, \ldots, m$ либо индекс группы $G_{i}$ в групе $G_{i-1}$ конечен, либо группа $G_{i-1} / G_{i}$ коммутативна.

Теорема 4.12 (Пикара-Вессио). Линейное дифференииальное уравнение над дифференциальным полем $K$ решается в квадратурах, в $k$-квадратурах или обобщенных квадратурах, если и только если группа Галуа уравнения над полем $K$ является соответственно разрешимой, $k$-разрешимой или почти разрешимой әруппой в категории алгебраических групп.

ЗАмЕчАнИЕ. В классической теореме Пикара-Вессио не обсуждается вопрос о разрешимости уравнений в $k$-квадратурах. Мы включили этот вопрос в теорему, потому что, во-первых, ответ на него аналогичен и, во-вторых, он переносится в топологический вариант теории Галуа.

В этом пункте мы докажем лишш необходимость условий на групп Галуа для разрешимости уравнения. Доказательство достаточности отложим до п. 4.7. Итак, справедлива следуюшая теорема.

ТЕОРема 4.13. Если линейное дифференциальное уравнение решается в квадратурах, в к-квадратурах или в обобщенных квадратурах, то группа Галуа $G$ этого уравнения является разрешимой, $k$-разрешимой или почти разрешимой группой в категории алгебраических групп.

ДокАЗАтельство. Разрешимость уравнений в обобшенных квадратурах над полем $K$ означает существование цепочки дифференщиальных полей $K=K_{0} \subset \cdots \subset$ $K_{N}$, в которой первое поле совпадает с исходньм полем $K$, последнее поле $K_{N}$ содержит все решения дифференциального уравнения и для каждого $i=1, \ldots, N$ поле $K_{i}$ получается из поля $K_{i-1}$ либо присоединением интеграла, либо присоединением экспоненты интеграла, либо присоединением всех решений алгебраического уравнения. (В случае разрешимости в квадратурах последний из этих типов расширений запрещен. В случае разрешимости в $k$-квадратурах допускается лишш присоединение корней алгебраических уравнений степени не вьше $k$.)

Пусть $G=G_{0} \supset \cdots \supset G_{m}=e-$ убывающая цепочка груп, в которой група $G_{i}$ - группа Галуа исходного уравнения над полем $K_{i}$. Согласно основной теореме 4.4 , фактор-группа $G_{i-1} / G_{i}$ является фактор-группой групшы Галуа расширения Пикара-Вессио $K_{i}$ поля $K_{i-1}$. Если это расширение является присоединением интеграла или экспоненты интеграла, то группа $G_{i-1} / G_{i}$ коммутативна как фактор-группа коммутативной группы (см. п. 4.4.2 и п. 4.4.3). Если расширение $K_{i}$ поля $K_{i-1}$ получено присоединением всех корней алгебраического уравнения, то фактор-группа $G_{i-1} / G_{i}$ конечна. Если это алгебраическое уравнение имеет степень $\leqslant k$, то между группами $G_{i} \supset G_{i-1}$ можно вставить цепочку нормальных делителей $G_{i}=G_{i 1} \supset \cdots \supset G_{i p}=$ $G_{i-1}$ такую, что глубина группы $G_{i j}$ в группе $G_{i, j-1}$ не превосходит $k$ (см. п. 3.4.2). Доказательство теоремы закончено.

Только что доказанную теорему можно сформулировать следуюшим образом.

Если расширение Пикара-Вессио является расширением Лиувилля, $k$-расширением Лиувилля или обобщенным расширением Лиувилля, то его группа Галуа является соответственно разрешимой, $k$-разрешимой или почти разрешимой группой в категории алгебраических групп. 
В такой переформулировке теорема становится применимой и к алгебраическим уравнениям над дифференциальными полями. Она дает более сильные результаты о неразрешимости алгебраических уравнений.

ТеОРема 4.14. Если группа Галуа алгебраического уравнения над дифференииальным полем $K$ неразрешима, то это алгебраическое уравнение не только не решается в радикалах, но не решается и в квадратурах. Если группа Галуа не является $k$-разрешимой, то алгебраическое уравнение не решается в $k$-квадратурах над $K$.

4.6. Алгебраические матричные группы и необходимые условия разрешимости. Группа Галуа линейного дифференщиального уравнения является алгебраической матричной группой. Такие группы обладают обшими свойствами, которые помогают переформулировать условия разрешимости, $k$-разрешимости и почти разрешимости группы Галуа и доказать, что эти условия являются достаточными (см. п. 4.7) для разрешимости уравнения.

Отметим прежде всего, что всякая алгебраическая матричная группа является группой Ли. Действительно, множество особых точек всякого алгебраического многообразия имеет коразмерность $\geqslant 1$. Но групповым преобразованием любая точка групшы переводится в любую другую точку. Поэтому около каждой точки группа устроена одинаково, и, следовательно, множество ее особых точек пусто. Компонента связности единичы алгебраической группь является нормальным делителем конечного индекса в этой әруппе. Действительно, компонента связности единицы является нормальньм делителем во всякой групе Ли, и каждое алгебраическое многообразие имеет лишь конечное число компонент связности.

Для дальнейшего ключевую роль играет следуюшая знаменитая теорема Ли, которую мы приведем без доказательства.

ТЕОРема 4.15 (Ли). Связная разрешимая матричная группа Ли в некотором базисе приводится к треугольному виду.

УТВЕРЖДЕНИЕ 4.16. Алгебраическая матричная группа является почти разрешимой группой в категории алгебраических групп, если и только если все матрицы ее компоненты связности единицы в некотором базисе одновременно приводятся к треугольному виду.

ДокАЗАТЕльство. Всякая группа, состоящая из треугольных матриц, разрешима. Это доказьвает утверждение в одну сторону. Пусть $G=G_{0} \supset \cdots \supset G_{n}=e$ - нормальная башня алгебраических подгрупп групшы $G$, для которой каждая фактор-группа $G_{i} / G_{i-1}$ либо коммутативна, либо конечна. Рассмотрим компоненты связности единищы этих групп. Они образуют нормальную башню $G^{0}=G_{0}^{0} \supset \cdots \supset$ $G_{n}^{0}=e$ алгебраических подгруп компоненты связности единицы $G^{0}$ группы $G$. При этом если фактор-группа $G_{i-1} / G_{i}$ коммутативна, то фактор-группа $G_{i-1}^{0} / G_{i}^{0}$ тоже коммутативна. Если фактор-группа $G_{i-1} / G_{i}$ конечна, то групшы $G_{i-1}^{0}$ и $G_{i}^{0}$ совпадают. Утверждение доказано.

УТВЕРЖДЕНИЕ 4.17. Алгебраическая матричная группа $G$ является разрешимой или k-разрешимой группой в категории алгебраических групп, если и только 
если все матрицы ее компоненты связности единицы $G^{0}$ в некотором базисе приводятся $к$ треугольному виду и конечная фактор-группа $G / G_{0}$ соответственно разрешима или $k$-разрешима.

ДокАЗАТЕЛЬСТВо. Согласно утверждению 4.16 , группа $G^{0}$ является треугольной. Кроме того, групп $G^{0}$ является нормальным делителем конечного индекса в групп $G$. Конечная фактор-группа $G / G_{0}$ является соответственно разрешимой или $k$-разрешимой группой. В обратную сторону утверждение очевидно.

В группах матриц есть замечательная топология Зарисского, сопоставляющая каждой группе $\Gamma \subset G L(V)$ ее алгебраическое замькание $\bar{\Gamma}$. Эта операция позволяет обобшить утверждения 4.16 и 4.17 на произвольные матричные групшы.

УТВЕРЖДЕНИЕ 4.18. 1) Матричная группа является обобщенно разрешимой группой, если и только если у нее существует треугольный нормальный делитель $Н$ конечного индекса. Матричная группа является $k$-разрешимой или разрешимой, если и только если конечная фактор-группа $G / H$ группы $G$ по некоторому треугольному нормальному делителю $Н$ конечного индекса является соответственно $k$-разрешимой или разрешимой группой.

2) Алгебраическая матричная группа $G$ является обобщенно разрешимой, $k$ разрешимой или разрешимой группой в категории алгебраических групп, если и только если она является соответственно обобщенно разрешимой, $k$-разрешимой или разрешимой әруппой.

ДокАЗАТЕЛЬство. Пусть $G=G_{0} \supset \cdots \supset G_{n}=e-$ нормальная башня групшы $G$, тогда замькания в топологии Зарисского групп из этой башни образуют нормальную башню алгебраических групп $\bar{G}=\bar{G}_{0} \supset \cdots \supset \bar{G}_{n}=e$. При этом если группа $G_{i-1} / G_{i}$ коммутативна, конечна или если групша $G_{i}$ имеет в $G_{i-1}$ глубину $\leqslant k$, то група $\bar{G}_{i-1} / \bar{G}_{i}$ будет соответственно коммутативной, конечной или группа $\bar{G}_{i}$ будет иметь в группе $\bar{G}_{i-1}$ глубину $\leqslant k$. Это доказьвает все пункты утверждения в одну сторону. В другую сторону все утверждения очевидны.

4.7. Достаточное условие разрешимости дифференциальных уравнений. Группу автоморфизмов Г дифференциального поля $F$ с дифференциальньм полем неподвижных элементов $K$ назовем допустимой группой автоморфизмов, если существует конечномерное пространство $V$ надполем констант, инвариантное относительно групшы Г и такое, что $K\langle V\rangle=F$. Согласно теории Пикара-Вессио (см. следствие 4.5), дифференциальное поле $F$ является расширением Пикара-Вессио дифференциального поля $K$, если и только если существует допустимая группа автоморфизмов дифференщиального поля $F$ с дифференциальным полем неподвижных элементов $K$. В обшем случае сушествование допустимой групшы преобразований для расширения Пикара-Вессио совсем неочевидно и является частью основной теоремы этой теории. Однако для обширного класса случаев сушествование допустимой группы автоморфизмов известно априори. Такой класс случаев доставляют расширения поля рациональных функций всеми решениями любого линейного дифференциального уравнения типа Фукса (см. п. 6.1.1). В этих случаях группа монодромии уравнения играет роль групшы Г.

Если группа Г разрешима, то элементы поля $F$ представляются в квадратурах через элементы поля $K$. Конструкция такого представления, по сушеству, относится 
к линейной алгебре и не использует основньх теорем Пикара-Вессио. Допустимая группа автоморфизмов Г изоморфна индуцированной групе линейных преобразований пространства $V$, и ее можно рассматривать как матричную группу.

ЛЕмма 4.19 (Лиувилль). Если все преобразования допустимой группы Г приводятся в некотором базисе к треугольному виду, то дифференциальное поле $F$ является расширением Лиувилля дифференииального поля $K$.

ДокАЗАТЕльство. Пусть $e_{1}, \ldots, e_{n}$ - базис пространства $V$, в котором каждое преобразование $\mu \in \Gamma$ имеет вид $\mu\left(e_{i}\right)=\sum_{j \leqslant i} a_{i j} e_{j}$. Рассмотрим векторное пространство $\widetilde{V}$, натянутое на векторы $\widetilde{e}_{i}=\left(\frac{e_{i}}{e_{1}}\right)^{\prime}$, где $i=2, \ldots, n$. Пространство $\widetilde{V}$ инвариантно относительно группы $\Gamma$, причем каждое преобразование $\mu$ групшы $Г$ в базисе $\widetilde{e}_{i}$ имеет треугольный вид. Действительно,

$$
\mu\left(\widetilde{e}_{i}\right)=\mu\left(\left[\frac{e_{i}}{e_{1}}\right]^{\prime}\right)=\left(\frac{a_{i 1}}{a_{11}}+\sum_{2 \leqslant j \leqslant i} \frac{a_{i j}}{a_{11}} \frac{e_{j}}{e_{1}}\right)^{\prime}=\sum_{2 \leqslant j \leqslant i} \frac{a_{i j}}{a_{11}} \widetilde{e}_{j} .
$$

Пространство $\widetilde{V}$ имеет меньшую размерность, чем пространство $V$, поэтому можно считать, что дифференциальное поле $K\langle\widetilde{V}\rangle$ есть расширение Лиувилля дифференщиального поля $K$. Для всякого $\mu \in \Gamma$ имеем $\mu\left(\frac{e_{1}^{\prime}}{e_{1}}\right)=\frac{a_{11} e_{1}^{\prime}}{a_{11} e_{1}}=\frac{e_{1}^{\prime}}{e_{1}}$, следовательно, элемент $\frac{e_{1}^{\prime}}{e_{1}}=a$ лежит в дифференциальном поле инвариантов $K$. Дифференциальное поле $F$ получается из дифференциального поля $K$ присоединением экспоненты интеграла $e_{1}$ от элемента $a$ и интегралов $\frac{e_{i}}{e_{1}}$ от элементов $\widetilde{e}_{i}$, где $i=2, \ldots, n$.

УТВЕРЖДЕНИЕ 4.20. Если әруппа Г допустимых автоморфизмов поля $F$ с полем неподвижных әлементов $K$ почти разрешима, то существует инвариантное относительно группы $\Gamma$ поле $K_{0}$ такое, что 1) поле $F$ является расширением Лиувилля поля $\left.K_{0}, 2\right)$ индуиированная группа автоморфизмов поля $K_{0}$ конечна, при этом каждый әлемент поля $K_{0}$ является алгебраическим над полем $K, 3)$ если группа Г разрешима, то каждый әлемент поля $K_{0}$ представим в радикалах над полем $K$.

ДокАЗАТЕЛЬСТво. Пусть $V$ - инвариантное пространство относительно групы Г такое, что $K\langle V\rangle=F$.

Из утверждения 4.16 вытекает, что группа Г обладает нормальным делителем $\Gamma_{0}$ конечного индекса, приводяшимся в некотором базисе пространства $V$ к треугольному виду. Пусть $K_{0}$ - дифференщиальное поле инвариантов групшы Г. Согласно лемме 4.19, дифференщиальное поле $F$ есть расширение Лиувилля дифференциального поля $K_{0}$.

Очевидно (см. утверждение 3.9 ), что поле $K_{0}$ инвариантно относительно действия групшы $Г$, и индуцированная групша автоморфизмов этого поля $\widetilde{\Gamma}_{0}$ является конечной фактор-группой групшы $Г$. Поэтому каждый элемент поля $K_{0}$ алгебраичен над $K$ (см. лемму 3.17). Если исходная группа $Г$ разрешима, то ее конечная фактор-группа $\widetilde{\Gamma}_{0}$ тоже разрешима. В этом случае любой элемент поля $K_{0}$ выражается в радикалах через элемент поля $K$ (см. п. 3.3.1).

Доказательство следуюшего утверждения опирается на теорию Галуа. 
УТВЕРЖДЕНИЕ 4.21. Если в условиях утверждения 4.20 группа $\Gamma \quad k$-разрешима, то каждый әлемент поля $K_{0}$ выражсатся через әлементы поля $K$ при помощи радикалов и решений алгебраических уравнений степени не выше $k$.

ДокАЗАТЕльство. Так как группа $\widetilde{\Gamma}_{0}$ конечна, то расширение $K_{0}$ поля $K$ является расширением Галуа поля $K$. Если группа $\Gamma_{0} k$-разрешима, то ее конечная фактор-группа тоже $k$-разрешима. Утверждение 4.21 теперь вытекает из теоремы 3.16.

Закончим теперь доказательство теоремы Пикара-Вессио (см. п. 4.5).

Согласно основной теореме 4.4 , для всякого линейного дифференциального уравнения над дифференциальньм полем $K$ его группа Галуа оставляет неподвижными лишь элементы поля $K$. Поэтому применимы только что доказанные утверждения 4.20 и 4.21, что и доказывает достаточность условий на группу Галуа в теореме Пикара-Вессио.

Теорема Пикара-Вессио не только доказьвает критерий Лиувилля-МордухайБолтовского (см. п. 2.3.2), но позволяет его обобщить на случай разрешимости в квадратурах и $k$-квадратурах. Именно, справедливы следующие утверждения.

Линейное дифференциальное уравнение $n$-го порядка решается в обобщенных квадратурах над дифференщиальным полем $K$, если и только если, во-первых, у него существует решение $y_{1}$, удовлетворяющее уравнению $y_{1}^{\prime}=a y_{1}$, где $a$ - элемент, принадлежаший некоторому алгебраическому расширению $K_{1}$, и, во-вторых, если дифференциальное уравнение $(n-1)$-го порядка на функцию $z=y^{\prime}-a y$ с коэффициентами из поля $K_{1}$, полученное из исходного уравнения процедурой понижения порядка (см. п. 4.1.2), решается в обобщенных квадратурах. Аналогичные утверждения справедливы и для разрешимости линейного дифференциального уравнения в квадратурах и в $k$-квадратурах. Для разрешимости в квадратурах надо дополнительно требовать, чтобы алгебраическое расширение $K_{1}$ получилось из $K$ присоединением радикалов, а для разрешимости в $k$-квадратурах - чтобы расширение $K_{1}$ получалось из $K$ присоединением радикалов и корней алгебраических уравнений степени $\leqslant k$. Для доказательства этих утверждений достаточно посмотреть на конструкцию решений дифференциальных уравнений.

Дифференщиальная алгебра позволяет существенно уточнить этот критерий. Для линейных дифференииальных уравнений, коэффициентами которых являются рациональные функции с рачиональными коэффичиентами, удается получить конечный алгоритм, позволяющий определить, решается ли уравнение в обобщенных квадратурах, и в случае существования решения найти его [37]. Алгоритм использует: 1 ) оценку степени расширения $K_{1}$ поля $K$, зависящую лиш от порядка уравнения и вытекающую из обших соображений теории групп (см. п. 6.2.2); 2) теорию нормальных форм линейных дифференциальных уравнений в окрестности особой точки; 3) теорию исключения для дифференциальных уравнений и неравенств от нескольких функций (найденную Зайденбергом и обобщающую теорему Тарского-Зайденберга на случай дифференциальных полей).

4.8. Другие виды разрешимости. Колчин дополнил теорему Пикара-Вессио [26]. Он рассмотрел задачи о разрешимости линейных уравнений отдельно в интегралах и отдельно в экспонентах интегралов и варианты этих задач, в которых допускаются алгебраические расширения. 
При определении расширений Лиувилля использовались три вида расширений: алгебраические расширения, присоединения интегралов и присоединения экспонент интегралов. Можно определить более частные виды разрешимости, используя в качестве “строительных кирпичиков" только некоторые из этих расширений (и используя лишь специальные алгебраические расширения). Перечислим основные варианты.

1) Разрешимость в интегралах.

2) Разрешимость в интегралах и радикалах.

3) Разрешимость в интегралах и алгебраических функциях.

4) Разрешимость в экспонентах интегралов.

5) Разрешимость в экспонентах интегралов и алгебраических функциях.

Расшифруем третье из этих определений.

Рассмотрим произвольную цепочку дифференциальных полей $K=K_{0} \subseteq \cdots \subseteq$ $K_{n}$, в которой каждое следуюшее поле $K_{i}, i=1, \ldots, n$, либо получается из предыдущего поля $K_{i-1}$ присоединением интеграла над $K_{i-1}$, либо является алгебраическим расширением поля $K_{i-1}$. Каждый элемент дифференциального поля $K_{n}$ по определению представим в интегралах и алгебраических функииях над полем $K$. Уравнение решается над полем $K$ в интегралах и алгебраических функциях, если каждое его решение представлено в интегралах и алгебраических функциях.

Аналогично расшифровываются и другие виды разрешимости 1)-5).

ЗАмечАния. 1) Отдельно рассматривать разрешимость в радикалах и экспонентах интегралов не надо, так как каждый радикал является экспонентой интеграла.

2) Вьше мы рассматривали спешиальные алгебраические расширения, полученные присоединением корней алгебраических уравнений степени не вьше $k$. Можно было бы, скажем, определить $k$-разрешимость в интегралах, комбинируя подобные алгебраические расширения с присоединениями интегралов. Мы этого не делаем, чтобы не загромождать текст и из-за отсутствия интересных примеров.

ОПРЕДЕлЕНИЕ 1. Скажем, что матричная група $G$ является специальной треугольной әруппой, если существует базис, в котором все матрищы группы $G$ одновременно приводятся к треугольному виду и все собственные числа каждой матрицы из групшы $G$ равны единице.

ОПРЕДЕЛЕНИЕ 2. Скажем, что матричная группа диагональна, если существует базис, в котором все матрищы группы диагональны.

ТЕОРема 4.22 (Колчина о разрешимости в интегралах). Линейное дифференциальное уравнение над дифференциальным полем $K$ решается в интегралах, $в$ интегралах и радикалах, в интегралах и алгебраических функииях, если и только если группа Галуа уравнения над $K$ соответственно является специальной треугольной группой, разрешима и содержит специальный треугольный нормальный делитель конечного индекса, содержит специальный треугольный нормальный делитель конечного индекса.

ТЕОРема 4.23 (Колчина о разрешимости в экспонентах интегралов). Линейное дифференциальное уравнение над дифференииальным полем $K$ решается в әкспонентах интегралов и в әкспонентах интегралов и алгебраических функииях, 
если и только если его группа Галуа над $K$ соответственно разрешима и содержит диагональный нормальный делитель конечного индекса, содержит диагональный нормальный делитель конечного индекса.

Несколько слов о доказательстве этих теорем. Группа Галуа присоединения интеграла антикомпактна (см. п. 4.4.2). Группа Галуа присоединения экспоненты интеграла квазикомпактна (см. п. 4.4.3). Колчин развил теорию антикомпактных и квазикомпактных алгебраических матричных групп. Вот одно несложное утверждение из этой теории.

УТВЕРЖДЕНИЕ 4.24 [26]. 1) Алгебраическая матричная группа квазикомпактна, если и только если каждая матрица группь приводится к диагональному виду. 2) Алгебраическая матричная группа антикомпактна, если и только если все собственные числа каждой матрицы группь равны единище.

Теория квазикомпактных и антикомпактных групп вместе с основной теоремой теории Пикара-Вессио позволили Колчину доказать его теоремы о разрешимости в интегралах и о разрешимости в экспонентах интегралов.

Разумеется, теоремы Колчина, так же как и теорема Пикара-Вессио, справедливы не только для линейных дифференциальных уравнений, но и для расширений Пикара-Вессио (каждое такое расширение порождено решениями линейного дифференциального уравнения). Сформулируем критерий для различных видов представимости всех элементов расширения Пикара-Вессио с треугольной группой Галуа. Этот критерий легко вытекает из теорем Колчина и теоремы Пикара-Вессио. Мы применим этот критерий в п. 6.2.3 при обсуждении различных видов разрешимости систем уравнений типа Фукса с мальми коэффициентами.

РАСШИРЕНИЕ С ТРЕУГОЛЬНОЙ ГРУППОЙ ГАЛУА (ср. с [26]). Пусть расширение Пикара-Вессио F дифференииального поля $K$ имеет треугольную группу Галуа. Тогда каждый әлемент поля $F$ :

1) представим в квадратурах над полем $K$;

2) представим в интегралах и алгебраических функииях или в интегралах и радикалах ${ }^{1}$ над полем $K$, если и только если собственнье числа всех матрич әруппь Галуа - корни из единиць;

3) представим в интегралах над полем $K$, если и только если все собственные числа всех матриц группь Галуа равны единиче;

4) представим в экспонентах интегралов и алгебраических функциях или в әкспонентах интегралов ${ }^{1}$ над полем $K$, если и только если группа Галуа диагональна;

5) представим в алгебраических функциях или в радикалах ${ }^{1}$ над полем $K$, если и только если группа Галуа диагональна, а все собственнье числа всех матрич группь Галуа - корни из единичь;

6) лехит в поле $K$, если и только если группа Галуа тривиальна.

\footnotetext{
${ }^{1}$ Эти виды разрешимости различаются, если не требовать треугольности группы Галуа.
} 


\section{$\S 5$. Одномерный топологический вариант теории Галуа}

5.1. Предварительные замечания. В п. 5.1.1 рассказьвается о теории Галуа для полей мероморфных функций на алгебраических кривых, которая имеет прозрачную геометрическую интерпретацию и тесно связана с одномерным топологическим вариантом теории Галуа. В п. 5.1.2 обсуждаются топологическая непредставимость функций в радикалах и топологическая неэлементарность эллиптических функций, доказанные В.И. Арнольдом. В п. 5.1.3 обсуждается идея топологического варианта теории Галуа и трудности в ее реализации.

5.1.1. Теория Галуа полей мероморфншхх функцийна алгебраических кривых. С алгебраической точки зрения в этом пункте идет речь о полях, являюшихся расширениями степени трансцендентности один поля комплексных чисел $\mathbb{C}$, которые конечно-порождены над $\mathbb{C}$. Теория Галуа для таких полей имеет простой геометрический смысл.

Прежде всего, такое поле изоморфно полю $P_{M}$ мероморфных функций на некоторой связной компактной римановой поверхности $M$, определенной с точностью до аналитического диффеоморфизма. Простейший пример такого поля доставляет поле рациональных функций одной комплексной переменной, т.е. поле мероморфных функций на сфере Римана.

Гомоморфизму таких полей $\tau: P_{M_{1}} \rightarrow P_{M_{2}}$, тождественному на подполях комплексных чисел, отвечает регулярное отображение $\rho: M_{2} \rightarrow M_{1}$ соответствующих римановых поверхностей такое, что $\rho^{*} f=\tau(f)$, где $f$ и $\tau(f)$ - мероморфные функции на $M_{1}$ и $M_{2}$. Если отображение $\rho$ непостоянно, то образ $\tau\left(P_{M_{1}}\right)=\rho^{*}\left(P_{M_{1}}\right)$ поля $P_{M_{1}}$ изоморфен полю $P_{M_{1}}$, а поле $P_{M_{2}}$ является конечным алгебраическим расширением своего подполя $\tau\left(P_{M_{1}}\right)=\rho^{*}\left(P_{M_{1}}\right)$.

Непостоянное аналитическое отображение $\rho: M_{2} \rightarrow M_{1}$ связной компактной римановой поверхности $M_{2}$ в связную компактную риманову поверхность $M_{1}$ задает конечное разветвленное накрытие над поверхностью $M_{1}$. Разветвленные накрытия $\rho_{1}: M_{2} \rightarrow M_{1}$ и $\rho_{2}: M_{3} \rightarrow M_{1}$ задают изоморфные расширения поля $P_{M_{1}}$, если и только если соответствуюшие разветвленные накрытия изоморфны, т.е. если существует обратимое аналитическое отображение $\rho: M_{2} \rightarrow M_{3}$, коммутирующее с проекциями, т.е. $\rho_{1}=\rho_{2} \circ \rho$. Таким образом, теория конечных алгебраических расширений полей рассматриваемого типа эквивалентна теории конечных разветвленных накрытий над компактньми римановыми поверхностями. В частности, теория конечных алгебраических расширений поля мероморфных функций эквивалентна теории конечнолистных разветвлений накрытий над сферой Римана.

Теперь подробнее о приведенных выше фактах и о связанной с ними геометрии. Каждое конечное алгебраическое расширение всякого поля порождается над этим полем одним элементом $y$, удовлетворяюшим некоторому неприводимому алгебраическому уравнению над исходным полем. Пусть расширение поля $P_{M}$ порождено элементом $y$, удовлетворяющим неприводимому уравнению

$$
y^{n}+r_{1} y^{n-1}+\cdots+r_{n}=0,
$$

в котором $r_{i}$ - мероморфные функции на $M$. В малой окрестности точки $a$ определены $n$ аналитических ростков $y_{1 a}, \ldots, y_{n a}$, удовлетворяюших уравнению (10). Уравнение (10) неприводимо, если и только если каждый из этих ростков $y_{i a}$ получается 
из любого другого ростка $y_{j a}$ аналитическим продолжением вдоль некоторой кривой, лежашей на поверхности $M$ (см. [14]). Рассмотрим риманову поверхность $M_{i}$ ростка $y_{i a}$ над поверхностью $M, \rho_{i}: M_{i} \rightarrow M$. Поверхность $M_{i}$ является связньм компактным многообразием. Поле мероморфных функций на $M_{i}$ порождено над полем $\rho_{i}^{*} P_{M}$ элементом $y_{i}$ (см. [14]). Неприводимость уравнения означает также, что разветвленные накрытия $\rho_{i}: M_{i} \rightarrow M$ и $\rho_{j}: M_{j} \rightarrow M$, соответствуюшие римановым поверхностям различных решений $y_{i a}$ и $y_{j a}$ уравнения (10), изоморфны как накрытия. Итак, каждому конечному алгебраическому расширению поля $P_{M}$, заданному с точностью до изоморфизма, мы сопоставили класс әквивалентных конечных разветвленных покрытий над $M$.

Построение обратного отображения, сопоставляющего каждому конечному разветвленному накрытию $\rho: M_{1} \rightarrow M$ над $M$ конечное расширение поля $M$, основано на теореме существования Римана. Согласно этой теореме, на всяком одномерном комплексном многообразии для любого конечного множества существует мероморфная функция, которая принимает разные значения в точках множества. Рассмотрим мероморфную функцию на $M_{1}$, принимающую различные значения в различных прообразах какого-либо регулярного значения $a \in M$ отображения $\rho$. Такая функция $y$ является многозначной алгебраической функщией на $M$ и порождает все поле мероморфных функций на $M_{1}$ над подполем $\rho^{*}\left(P_{M}\right)$.

Перейдем к геометрическому описанию разветвленных накрытий над компактной римановой поверхностью $M$. Для задания такого накрытия достаточно фиксировать конечное множество $A \subset M$ и подгруппу $F$ конечного индекса в фундаментальной групе $\pi_{1}(M \backslash A)$ дополнения к множеству $A$. По этим данньм разветвленное накрытие над $M$ строится так. Сначала по подгруппе $F$ строится накрытие над множеством $M \backslash A$, образ фундаментальной группы которого при проекции в $M \backslash A$ совпадает с подгруппой $F$ (см., например, [12]). Число прообразов любой точки в $M \backslash A$ при этом накрытии совпадает с индексом подгруппы $F$ в группе $\pi_{1}(M \backslash A)$. Полученное накрытие однозначно компактифицируется добавлением некоторых точек, лежащих над множеством $A$ (множество добавленных точек над $a \in A$ находится в соответствии с множеством циклов в перестановке листов накрытия, соответствующих обходу вокруг точки $a)$.

Два разветвленных накрытия, построенных по множествам $A_{1} \subset M$ и $A_{2} \subset M$ и по группам $F_{1} \subset \pi_{1}\left(M \backslash A_{1}\right)$ и $F_{2} \subset \pi_{1}\left(M \backslash A_{2}\right)$, изоморфны, если и только если для конечного множества $B$, содержащего множества $A_{1}$ и $A_{2}$, подгруппы $\widetilde{F}_{1}, \widetilde{F}_{2}$ групшы $\pi_{1}(M \backslash B)$ сопряжены в этой группе $\pi_{1}(M \backslash B)$, где $\widetilde{F}_{1}$ и $\widetilde{F}_{2}-$ прообразы групп $F_{1}$ и $F_{2}$ при индуцированных вложениями гомоморфизмах групшы $\pi_{1}(M \backslash B)$ в групшы $\pi_{1}\left(M \backslash A_{1}\right)$ и $\pi_{1}\left(M \backslash A_{2}\right)$. Легко видеть, что вьписанное условие не зависит от выбора конечного множества $B$, содержашего множества $A_{1}$ и $A_{2}$.

Разветвленное накрытие $\rho: M_{1} \rightarrow M$ соответствует расширению Галуа поля $P_{M}$, если и только если определяюшая это накрытие подгруппа $H$ конечного индекса в $\pi_{1}(M \backslash A)$ является нормальной подгруппой в $\pi_{1}(M \backslash A)$ (здесь $A$ - любое конечное множество, содержашее все критические значения отображения $\rho$; вьписанное условие не зависит от конкретного выбора множества $A$ ). Группа Галуа этого расширения Галуа совпадает с фактор-группой $\pi_{1}(M \backslash A) / H$, изоморфной группе взачмно однозначных преобразований поверхности $M_{1}$ в себя, коммутирующих с проекиией $\rho$. 
Промежуточному расширению поля $P_{M}$ соответствует промежуточное разветвленное накрытие, т.е. такое накрытие $\rho_{2}: M_{2} \rightarrow M$, что сушествует отображение $\rho_{1}: M_{1} \rightarrow M_{2}$, для которого $\rho_{2} \circ \rho_{1}=\rho$. Промежуточное накрытие соответствует промежуточной подгруппе $F$ в $\pi_{1}(M \backslash A)$, т.е. такой подгрупше $F$, что $H \subseteq F$.

Промежуточное поле является расширением Галуа, если и только если $F$ - нормальный делитель в $\pi_{1}(M \backslash A)$. Группа Галуа промежуточного расширения Галуа является фактор-группой группы Галуа исходного расширения, так как фактор-группа $\pi_{1}(M \backslash A) / H$ естественно отображается на фактор-группу $\pi_{1}(M \backslash A) / F$.

Вьше мы привели геометрическую интерпретацию основной теоремы теории Галуа для полей мероморфных функций на алгебраических кривых. Нам осталось лишш описать геометрически поведение группы Галуа алгебраического уравнения при расширении основного поля. Итак, пусть $\rho_{1}: M_{1} \rightarrow M$ - разветвленное накрытие, соответствующее расширению Галуа поля $P_{M}$, и пусть $\rho_{2}: M_{2} \rightarrow M-$ другое накрытие, соответствуюшее другому расширению Галуа того же поля $P_{M}$. Пусть $B$ - любое конечное множество на $M$, содержащее критические точки отображений $\rho_{1}$ и $\rho_{2}$, и $G=\pi_{1}(M \backslash B)$ - фундаментальная группа дополнения к множеству $B$. Накрытия $\rho_{1}: M_{1} \backslash B_{1} \rightarrow M \backslash B$ и $\rho_{2}: M_{2} \backslash B_{2} \rightarrow M \backslash B$, где $B_{1}=\rho_{1}^{-1}(B)$ и $B_{2}=\rho_{2}^{-1}(B)$, соответствуют нормальным подгруппам $H_{1}$ и $H_{2}$ групшы $G$, изоморфным фундаментальным группам многообразий $M_{1} \backslash B_{1}$ и $M_{2} \backslash B_{2}$. Проекщия $\rho_{2}: M_{2} \backslash B_{2} \rightarrow M \backslash B$ отображает многообразие $M_{2} \backslash B_{2}$ в базу накрытия $\rho_{1}: M_{1} \backslash B_{1} \rightarrow M \backslash B$. При помоши отображения $\rho_{2}$ из накрытия $\rho_{1}: M_{1} \backslash B_{1} \rightarrow S \backslash B$ индуцируется накрытие $\rho: U \rightarrow M_{2} \backslash B_{2}$ над $M_{2} \backslash B_{2}$. Многообразие $U$, вообще говоря, несвязно. Каждая компонента связности $U_{i} \subset U$ определяет накрытие $\rho: U_{i} \rightarrow M_{2} \backslash B_{2}$. Легко видеть, что все накрытия, связанные с различньми компонентами $U_{i}$, эквивалентны как накрытия, и каждое из них соответствует нормальному делителю $H_{1} \cap H_{2}$ в групе $H_{2}$. Рассмотрим любую из компонент $U_{i}=V$. Накрытие $\rho: V \rightarrow M_{2} \backslash B_{2}$ допускает компактификацию $\rho: M_{3} \rightarrow M_{2}$, где $V=M_{3} \backslash B_{3}$ и $B_{3}=\rho^{-1}\left(B_{2}\right)$, соответствующую исходному расширению Галуа над полем $P_{M_{2}}$. Группа Галуа этого накрытия есть фактор-группа $H_{3} / H_{1} \cap H_{2}$. Именно это и утверждается в теореме 3.4 .

Итак, все утверждения теории Галуа для полей мероморфных функций на кривых имеют прозрачное геометрическое объяснение. Более того, геометрическая конструкиия разветвленных накрытий, соединенная с теоремой существования Римана, доставляет полное описание всех конечных расширений рассматриваемого класса полей. Например, она моментально решает для таких полей обратную задачу теории Галуа (т.е. задачу построения расширения Галуа с заданной группой Галуа). Отметим, что для поля рациональных чисел обратная задача теории Галуа до сих пор не решена.

5.1.2. Топологическая непредставимость функций в радикалах. Прежде чем переходить к топологическому варианту теории Галуа, остановимся еще на одной топологической интерпретации группы Галуа алгебраического уравнения над полем рациональных функций.

Пусть

$$
y^{n}+r_{1} y^{n-1}+\cdots+r_{n}=0
$$

- неприводимое уравнение над полем рациональных функций $P_{S}, r_{i} \in P_{S}$. 
Хорошо известно следующее утверждение.

УТВЕРЖДЕНИЕ 5.1. Группа Галуа уравнения (11) над полем рациональных функиий изоморфна группе монодромии (многозначной) алгебраической функиии $y$, определенной уравнением (11).

ДокАЗАТЕЛЬСТВо. Пусть $D$ - дискриминант уравнения (11). Обозначим через $[D]$ множество нулей и полюсов дискриминанта $D$. В малой связной окрестности $U$ точки $a, a \notin[D]$, на сфере Римана определены $n$ голоморфных решений $y_{1 a}, \ldots, y_{n a}$ уравнения (11). Обозначим через $Q$ поле мероморфных функций в области $U$, полученное присоединением к полю $P_{S}$ всех этих решений. Пусть $\gamma-$ кривая на сфере Римана, начинающаяся и заканчивающаяся в точке $a$ и не пересекаюшая множество $[D]$. В доль кривой $\gamma$ регулярно продолжается каждое решение $y_{1 a}, \ldots, y_{n a}$ уравнения (11), поэтому вдоль $\gamma$ мероморфно продолжается любой элемент $z$ поля $Q$. Отображение $z \rightarrow z(\gamma)$, сопоставляющее элементу $z$ его мероморфное продолжение вдоль $\gamma$, является, очевидно, изоморфизмом поля $Q$. Этот изоморфизм однозначно определяется перестановкой $y_{i a} \rightarrow y_{i a}(\gamma)$ элементов $y_{1 a}, \ldots, y_{n a}$, зависит лиш от класса $[\gamma]$ кривой $\gamma$ в $\pi_{1}(S \backslash[D], a)$ и оставляет тождественно на месте все рациональные функции. Обратно, элементы поля $Q$, остающиеся на месте при всех изоморфизмах $z \rightarrow z(\gamma)$, являются однозначными алгебраическими функциями, т.е. являются рациональньми функциями. Следовательно, группа изоморфизмов вида $z \rightarrow z(\gamma)$ поля $Q$ совпадает с его групой Галуа над полем $P_{S}$. С другой стороны, эта группа изоморфина группе перестановок листов $y_{1 a}, \ldots, y_{n a}$ алгебраической функции $y$, соответствующих аналитическим продолжениям $y_{i a} \rightarrow y_{i a}(\gamma)$ вдоль путей $\gamma, \gamma \in \pi_{1}(S \backslash D, a)$, т.е. изоморфна групе монодромии функции $y$. Утверждение доказано.

Из теории Галуа и только что доказанного утверждения вытекает следующее следствие.

СлЕДСтВИЕ 5.2. 1) Алгебраическая функиия представима в радикалах, если и только если ее группа монодромии разрешима. 2) Алгебраическая функция представима в к-радикалах, если и только если ее группа монодромии $k$-разрешима.

В.И. Арнольд доказал топологическую неразрешимость ряда классических задач [2]-[9]. Приведем принадлежашее Арнольду определение.

ОПРЕДЕЛЕНИЕ (Арнольд). $f: X \rightarrow Y$ назьвается топологически нехорошим отображением (например, топологически неэлементарной функцией), если среди (лево-право) топологически эквивалентных ему нет хороших (элементарных, например).

С каждой многозначной аналитической функцией $f$ комплексного переменного связаны ее риманова поверхность $M_{f}$ и проекция $\pi_{f}: M_{f} \rightarrow S^{2}$ этой поверхности на сферу Римана $S^{2}$.

СлЕДСТВИЕ 5.3. Пусть проекиии $\pi_{f} u \pi_{g}$ римановых поверхностей $M_{f} u M_{g}$ функиий $f$ и $g$ на сферу Римана топологически әквивалентнь. Тогда функиии $f$ и $g$ представимы или непредставимы в радикалах (k-радикалах) одновременно (т.е. топологический тип проекции римановой поверхности функции на сферу Римана отвечает за представимость функции в радикалах и в $k$-радикалах). 
ДокАЗАТЕльство. Следствие 5.3 немедленно вытекает из следствия 5.2. Действительно, алгебраичность функции связана с компактностью ее римановой поверхности, ее представимость в радикалах связана с разрешимостью ее группы монодромии, а ее представимость в $k$-радикалах связана с $k$-разрешимостью ее группы монодромии. Все эти свойства топологические.

Арнольд в 60-е годы доказал топологическую непредставимость в радикалах обших алгебраических функций (см. следствие 5.3) непосредственно топологически, не опираясь на теорию Галуа, и прочел курс на эту тему в Колмогоровском интернате. В. А. Алексеев значительно переработал этот курс и опубликовал его в виде книги [1]. Согласно Арнольду, топологическое доказательство неразрешимости какой-либо задачи, как правило, влечет за собой новые следствия. Например, из топологического доказательства непредставимости в радикалах функции с неразрешимой группой монодромии легко вытекает непредставимость такой функции формулой, включающей в себя не только радикалы, но и любые целые функции [19].

В 60-е годы Арнольд нашел также результаты о топологической неэлементарности эллиптических функций и интегралов (и других близких вещей), но ничего не опубликовал на эту тему. В декабре 2003 года Владимир Игоревич написал мне письмо об этом. Следуюшая теорема, так же как и приведенное выше определение, заимствованы из этого письма.

Теорема 5.4 (Арнольд). Если мероморфная функиия $g: U \rightarrow \mathbb{C} P^{1}$, определенная в комплексной области $U \subset \mathbb{C}$, топологически әквивалентна әллиптической функиии $f: \mathbb{C} \rightarrow \mathbb{C} P^{1}$, то $g$ - әллиптическая функиия (возможсно, с иными, нежели у функиии $f$, периодами).

ДокАЗАТЕльствО. Эллиптическая функция $f$ инвариантна относительно групшы сдвигов $\mathbb{Z}^{2}\left(z \rightarrow z+k_{1} w_{1}+k_{2} w_{2},\left(k_{1}, k_{2}\right) \in \mathbb{Z}^{2}\right)$. Поэтому функщия $g$ инвариантна относительно групшы гомеоморфизмов $\mathbb{Z}^{2}$ области $U$. Каждый гомеоморфизм $h$ этой групшы, на самом деле, является биголоморфньм отображением области $U$ в себя. Действительно, по теореме об обратной функции из тождества $g(z) \equiv g(h(z))$ вытекает голоморфность отображения $h$ в окрестности каждой точки, не принадлежащей прообразу при отображении $h$ множества критических точек функции $g$. В точках этого прообраза отображение $h$ голоморфно по теореме об устранимой особенности. По условию область $U$ гомеоморфна $\mathbb{C}$. Следовательно, по теореме Римана область $U$ либо совпадает с $\mathbb{C}$, либо биголоморфно эквивалентна внутренности единичного круга. Область $U$ совпадает с $\mathbb{C}$, так как группа биголоморфных преобразований единичного круга не содержит подгрупшы $\mathbb{Z}^{2}$. Подгруппа $\mathbb{Z}^{2}$ групшы биголоморфных преобразований $\mathbb{C}$, действующая на $\mathbb{C}$ без неподвижных точек, является группой сдвигов $\left(z \rightarrow k_{1} \tau_{1}+k_{2} \tau_{2},\left(k_{1}, k_{2}\right) \in \mathbb{Z}^{2}\right)$. Поэтому $g$ - эллиптическая функция.

Как известно, эллиптические функции неэлементарны ${ }^{2}$. Из этого классического

\footnotetext{
${ }^{2}$ Неэлементарность эллиптических функций вытекает из обобщения теории Пикара-Вессио, принадлежащего Колчину [27]. Это обобщение применимо не только к линейным, но и к некоторым нелинейным дифференциальным уравнениям, например к уравнению на $\gamma$-функцию Вейерштрасса. Группа Галуа дифференциального поля эллиптических функций над полем констант $\mathbb{C}$, очевидно, содержит группу $\mathbb{C} / \mathbb{Z}^{2}$, состоящую из фактор-группы группы сдвигов $f(z) \rightarrow f(z+a)$ по подгруппе $\mathbb{Z}^{2}$ периодов эллиптических функций. (Несложно показать, что группа Галуа сов-
} 
результата и доказанной выше теоремы вытекает топологическая неэлементарность эллиптических функций.

Ниже приводится цитата из письма Владимира Игоревича.

“Эти соображения доказьвали, мне помнится, топологическую неэлементарность как эллиптических функций, $f$, так и эллиптических интегралов, $f^{-1}$, да и многого другого. Причем все это обобщается и на кривые других родов (с другими накрытиями, или хотя бы с универсальными накрытиями). Не помню, доказал ли я аккуратно, но думаю, что у меня были соображения, почему аналогичные приведенной теореме многомерные высказьвания должны быть неверны: помнится, что сохранение топологического типа в многомерном случае не гарантирует сохранение алгебраичности. Само по себе это не препятствует топологической неэлементарности (нехорошести), но препятствует моему доказательству его, доказательству путем сведения к неэлементарности классического (алгебраического) объекта вроде эллиптической функции".

5.1.3. Об одномерном топологическом варианте теории Галуа. На классе алгебраических функщий група монодромии совпадает с группой Галуа и отвечает за представимость функции в радикалах (см. п. 5.1.2). Но группа монодромии определена не только для алгебраических функций, она определена для логарифма, арктангенса и для многих других функций, для которых групп Галуа не определена. Естественно попытаться для таких функций использовать группу монодромии вместо групшы Галуа для доказательства непринадлежности функции тому или иному классическому классу. Именно этот подход и реализует топологический вариант теории Галуа [18]-[22].

Приведем пример, которьй показьвает, какие трудности приходится преодолевать на этом пути.

Рассмотрим элементарную функцию $f$, определенную следующей формулой:

$$
f(z)=\ln \left(\sum_{j=1}^{n} \lambda_{j} \ln \left(z-a_{j}\right)\right)
$$

где $a_{j}, j=1, \ldots, n,-$ различные точки на комплексной прямой и $\lambda_{j}, j=1, \ldots, n,-$ комплексные константы. Обозначим через $\Lambda$ аддитивную группу комплексных чисел, порожденную константами $\lambda_{1}, \ldots, \lambda_{n}$. Ясно, что если $n>2$, то почти для всякого набора констант $\lambda_{1}, \ldots, \lambda_{n}$ групша $\Lambda$ всюду плотна на комплексной прямой.

УТВЕРЖДЕНИЕ 5.5. Если группа $\Lambda$ всюду плотна на комплексной прямой, то әлементарная функция $f$ имеет всюду плотное множество точек логарифмического ветвления.

ДОкАЗАТЕЛЬСТВо. Пусть $g_{a}$ - один из ростков функции $g$, определенной формулой $g(z)=\sum_{j=1}^{n} \lambda_{j} \ln \left(z-a_{j}\right)$ в точке $a, a \neq a_{j}, j=1, \ldots, n$. После обхода точек $a_{1}, \ldots, a_{n}$ к ростку $g_{a}$ прибавится число $2 \pi i \lambda$, где $\lambda$ - элемент групшы $\Lambda$. Обратно,

падает с этой группой.) Непредставимость эллиптических функций в обобщенных квадратурах, согласно Колчину, вытекает из несуществования у группы $\mathbb{C} / \mathbb{Z}^{2}$ нормальной башни подгрупп, каждая фактор-группа относительно которой является или конечной группой, или аддитивной, или мультипликативной группой комплексных чисел. 
всякий росток $g_{a}+2 \pi i \lambda$, где $\lambda \in \Lambda$, получается из ростка $g_{a}$ в результате аналитического продолжения вдоль некоторой кривой. Пусть $U$ - малая окрестность точки $a$ и $G: U \rightarrow C$ - аналитическая функция, росток которой в точке $a$ есть $g_{a}$. Образ $V$ области $U$ при отображении $G: U \rightarrow \mathbb{C}$ открыт. Поэтому в области $V$ найдется точка вида $2 \pi i \lambda$, где $\lambda \in \Lambda$. Функция $G-2 \pi i \lambda$ является одной из ветвей функции $g$ над областью $U$, причем множество нулей этой ветви в области $U$ непусто. Поэтому одна из ветвей функции $f=\ln g$ имеет в $U$ точку логарифмического ветвления.

Несложно проверить, что в условиях утверждения группа монодромии функции $f$ континуальна (это неудивительно: фундаментальная группа $\pi_{1}(S \backslash A)$, где $A$-счетное всюду плотное множество на сфере Римана, очевидно, содержит континуум элементОв).

Можно показать также, что образ фундаментальной группы $\pi_{1}\left(S^{2} \backslash\{A \cup b\}\right)$, где $b \notin A$ - любая точка на комплексной прямой, в группе перестановок листов функции $f$ является собственной подгруппой групш монодромии функции $f$. (То обстоятельство, что удаление одной лишней точки может изменить группу монодромии, несколько усложняет все доказательства.)

Итак, уже простейшие элементарные функции могут иметь всюду плотное множество особых точек и континуальную группу монодромии.

В топологическом варианте теории Галуа мы рассматриваем функции, представимые в квадратурах, как многозначные аналитические функции одного комплексного переменного. Оказывается, что существуют топологические ограничения на характер расположения над комплексной плоскостью римановой поверхности функции, представимой в квадратурах. Если функция не удовлетворяет этим ограничениям, то ее нельзя выразить в квадратурах.

Этот подход, кроме геометрической наглядности, имеет следуюшее преимушество. Топологические запреты относятся к характеру многозначности функции. Они сохраняются не только для функций, представимых в квадратурах, но и для значительно более широкого класса функций. Этот более широкий класс получится, если к функциям, представимым в квадратурах, добавить все мероморфные функции и разрешить им участвовать во всех формулах. Из-за этого топологические результаты о непредставимости в квадратурах оказьваются более сильными, чем алгебраические. Дело в том, что суперпозищия функций - не алгебраическая операция. В дифференциальной алгебре вместо суперпозиции функций рассматривается дифференциальное уравнение, которому она удовлетворяет. Но, например, Г-функция Эйлера не удовлетворяет никакому алгебраическому дифференциальному уравнению. Поэтому безнадежно искать уравнение, которому удовлетворяет, скажем, функщия $\Gamma(\exp x)$. Единственные известные результаты о непредставимости функций в квадратурах и, скажем, в Г-функциях Эйлера получены только нашим методом.

С другой стороны, этим методом невозможно доказать непредставимость в квадратурах какой-либо однозначной мероморфной функции.

Используя дифференциальную теорию Галуа (а точнее, ее линейно-алгебраическую часть, имеющую дело с алгебраическими матричными групами и их дифференциальными инвариантами), можно показать, что единственные причины неразрешимости в квадратурах линейных дифференциальных уравнений типа Фукса - топологические (ср. с §6). Другими словами, если к разрешимости в квадратурах дифферен- 
циального уравнения типа Фукса не существует топологических препятствий, то оно решается в квадратурах.

Существуют следующие топологические препятствия к представимости функций в квадратурах, в обобщенных квадратурах и в $k$-квадратурах.

Во-первых, функции, представимые в обобщенных квадратурах, и, в частности, функции, представимые в квадратурах и в $k$-квадратурах, могут иметь не более чем счетное число особых точек на комплексной прямой (см. п. 5.2). (Хотя уже для простейших функций, представимых в квадратурах, множество особых точек может быть всюду плотным.)

Во-вторых, группа монодромии функции, представимой в квадратурах, обязательно разрешима (см. п. 5.5.2). (Хотя уже для простейших функций, представимых в квадратурах, групша монодромии может содержать континуум элементов.)

Аналогичные ограничения на расположение римановой поверхности существуют и для функций, представимых в обобшенных квадратурах и в $k$-квадратурах. Однако эти ограничения формулируются сложнее. В них группа монодромии фигурирует не как абстрактная группа, а как група перестановок множества листов функции. Или, другими словами, в этих ограничениях фигурирует не только группа монодромии, но и монодромная пара функции, состоящая из ее группы монодромии и стационарной подгруппы некоторого ростка (см. п. 5.3.3).

Переходим к подробному описанию этого геометрического подхода к проблеме разрешимости.

5.2. Функции с не более чем счетным множеством особых точек. В этом пункте определяется обширный класс функций одной комплексной переменной, нужньй для построения топологического варианта теории Галуа.

5.2.1. Запрешенные множества. Определим класс функций, внутри которого будут проводиться дальнейшие рассмотрения. Многозначная аналитическая функция одного комплексного переменного называется $\mathcal{S}$-функиией, если множество ее особых точек не более чем счетно. Уточним это определение.

Два регулярных ростка $f_{a}$ и $g_{b}$, заданных в точках $a$ и $b$ сферы Римана $S^{2}$, называются эквивалентньми, если росток $g_{b}$ получается из ростка $f_{a}$ регулярным продолжением вдоль некоторой кривой. Каждый росток $g_{b}$, эквивалентный ростку $f_{a}$, называется также регулярньм ростком многозначной аналитической функции $f$, порожденной ростком $f_{a}$.

Точка $b \in S^{2}$ назьвается особой для ростка $f_{a}$, если сушествует кривая $\gamma:[0,1] \rightarrow$ $S^{2}, \gamma(0)=a, \gamma(1)=b$, такая, что росток не может быть регулярно продолжен вдоль этой кривой, но для любого $t, 0 \leqslant t<1$, росток регулярно продолжается вдоль укороченной кривой $\gamma:[0, t] \rightarrow S^{2}$. Легко видеть, что у эквивалентных ростков множества особых точек совпадают.

Регулярньй росток назьвается $\mathcal{S}$-ростком, если множество его особых точек не более чем счетно. Многозначная аналитическая функция назьвается $\mathcal{S}$-функцией, если каждый ее регулярньй росток является $\mathcal{S}$-ростком.

В дальнейшем нам понадобится лемма, согласно которой малым шевелением кривую на плоскости можно снять со счетного множества.

ЛЕмма 5.6 (о снятии кривой со счетного множества). Пусть $A-$ не более чем счетное множсество на плоскости $\mathbb{C}, \gamma:[0,1] \rightarrow \mathbb{C}-$ кривая и $\varphi-$ непрерывная 
положительная функиия на интервале $0<t<1$. Тогда существует кривая $\widehat{\gamma}:[0,1] \rightarrow \mathbb{C}$ такая, что при $0<t<1 \widehat{\gamma}(t) \notin A u|\gamma(t)-\widehat{\gamma}(t)|<\varphi(t)$.

"Научное" доказательство леммы заключается в следующем. В функциональном пространстве кривых $\bar{\gamma}$, близких к кривой $\gamma,|\gamma(t)-\bar{\gamma}(t)|<\varphi(t)$, кривые, не пересекающие одной из точек множества $A$, образуют открытое плотное множество. Пересечение счетного числа открытых плотных множеств в таких функциональных пространствах непусто.

Приведем элементарное доказательство леммы (оно почти дословно переносится на более общий случай, когда множество $A$ несчетно, но имеет нулевую длину по Хаусдорфу, ср. с п. 5.5.3). Сначала построим бесконечнозвенную ломаную $\bar{\gamma}$ с вершинами, не принадлежашими $A$, такую, что $|\gamma(t)-\bar{\gamma}(t)|<\frac{1}{2} \varphi(t)$. Такую ломаную можно построить, так как дополнение к множеству $A$ всюду плотно. Покажем, как изменить каждое звено $[p, q]$ ломаной $\bar{\gamma}$, чтобы она не пересекала множества $A$. Возьмем отрезок $[p, q]$. Пусть $m-$ перпендикуляр к его середине. Рассмотрим двузвенные ломаные $[p, b]$, $[b, q]$, где $b \in m$ и точка $b$ достаточно близка к отрезку. Они пересекаются только по концам $p, q$, и их число континуально. Значит, среди них есть ломаная, не пересекающая множества $A$. Заменив таким образом каждое звено бесконечнозвенной ломаной, получим искомую кривую.

Кроме множества особых точек удобно рассматривать и другие множества, вне которых функция неограниченно продолжается. Не более чем счетное множество $A$ называется запрещенным множеством для регулярного ростка $f_{a}$, если росток $f_{a}$ регулярно продолжается вдоль кривой $\gamma(t), \gamma(0)=a$, пересекаюшей множество $A$ лиш, может быть, в начальньй момент.

ТеОРема 5.7 (о запрешенном множестве). Не более чем счетное мнохсество является запрещенным множеством ростка, если и только если оно содержит множество его особых точек. В частности, росток обладает некоторым запрещенным множеством, если и только если он является ростком $\mathcal{S}$-функции.

ДокАЗАТЕЛЬство. Предположим, что сушествует особая точка $b$ ростка $f_{a}$, не лежашая в некотором запрешенном множестве $A$ этого ростка. По определению должна сушествовать кривая $\gamma:[0,1] \rightarrow S^{2}, \gamma(0)=a, \gamma(1)=b$, вдоль которой не сушествует регулярного продолжения ростка $f_{a}$, но вдоль которой росток продолжается до любой точки $t<1$. Не ограничивая общности, можно считать, что точки $a, b$ и кривая $\gamma(t)$ лежат в конечной части сферы Римана, т.е. $\gamma(t) \neq \infty$ при $0 \leqslant t \leqslant 1$. Обозначим через $R(t)$ радиус сходимости рядов $f_{\gamma(t)}$, получающихся при продолжении ростка $f_{a}$ вдоль кривой $\gamma(t)$. Функция $R(t)$ непрерьвна на полуинтервале [0,1). Согласно лемме 5.6, существует кривая $\widehat{\gamma}(t), \widehat{\gamma}(0)=a, \widehat{\gamma}(1)=b$, такая, что $|\gamma(t)-\widehat{\gamma}(t)|<\frac{1}{3} R(t)$ и $\widehat{\gamma}(t) \notin A$ при $t>0$. Росток $f_{a}$ по условию должен продолжаться вдоль кривой $\widehat{\gamma}$ до точки 1 . Но отсюда легко следует, что росток $f_{a}$ продолжается вдоль кривой $\gamma$. Полученное противоречие доказьвает, что множество особых точек ростка $f_{a}$ содержится во всяком запрещенном множестве этого ростка. Обратное утверждение (счетное множество, содержашее множество особенностей ростка, является запрешенным для ростка) очевидно.

5.2.2. Замкнутость класса $\mathcal{S}$-функций. Докажем замкнутость введенного класса функций относительно всех естественных операций. 
Теорема 5.8 (о замкнутости класса $\mathcal{S}$-функший). Класс $\mathcal{S}$ всех $\mathcal{S}$-функций замкнут относительно следующих операций:

1) дифферениирования, т.е. если $f \in S$, mо $f^{\prime} \in \mathcal{S}$;

2) интегрирования, т.е. если $f \in \mathcal{S}$ и $g^{\prime}=f$, mо $g \in \mathcal{S}$;

3) суперпозиции, т.е. если $g, f \in \mathcal{S}$, mо $g \circ f \in \mathcal{S}$;

4) мероморфных операций, т.е. если $f_{i} \in \mathcal{S}, i=1, \ldots, n, F\left(x_{1}, \ldots, x_{n}\right)$ мероморфная функиия п-переменных и $f=F\left(f_{1}, \ldots, f_{n}\right)$, mо $f \in \mathcal{S}$;

5) решения алгебрачческих уравнений, т.е. если $f_{i} \in \mathcal{S}, i=1, \ldots, n, u f^{n}+$ $f_{1} f^{n-1}+\cdots+f_{n}=0$, mo $f \in \mathcal{S}$

6) решения линейных дифференциальных уравнений, т.е. если $f_{i} \in \mathcal{S}, i=$ $1, \ldots, n, u f^{(n)}+f_{1} f^{(n-1)}+\cdots+f_{n}=0$, mo $f \in \mathcal{S}$.

ДокАЗАтЕльство. 1)-2). Пусть $f_{a}, a \neq \infty,-$ росток $\mathcal{S}$-функции с множеством особых точек $A$. Если росток $f_{a}$ регулярно продолжается вдоль некоторой кривой $\gamma$, лежащей в конечной части сферы Римана, то интеграл и производная этого ростка регулярно продолжаются вдоль кривой $\gamma$. Поэтому в качестве запрешенного множества для интеграла и производной ростка $f_{a}$ достаточно взять множество $A \cup\{\infty\}$.

3) Пусть $f_{a}$ и $g_{b}$ - ростки $\mathcal{S}$-функций с множествами особых точек $A$ и $B$ и $f_{a}(a)=b$. Обозначим через $f^{-1}(B)$ полный прообраз множества $B$ при многозначном соответствии, порожденном ростком $f_{a}$. Иными словами, $x \in f^{-1}(B)$, если и только если сушествует росток $\psi_{x}$, эквивалентньй ростку $f_{a}$ и такой, что $\psi(x) \in B$. Множество $f^{-1}(B)$ не более чем счетно. В качестве запрешенного множества ростка $g_{b} \circ f_{a}$ достаточно взять множество $A \cup f^{-1}(B)$.

4) Пусть $f_{i a}$ - ростки $\mathcal{S}$-функций, $A_{i}$ - множества их особых точек и $F$ - мероморффная функция $n$ переменных. Мы предполагаем, что ростки $f_{i a}$ и функция $F$ таковы, что росток $f_{a}=F\left(f_{1 a}, \ldots, f_{n a}\right)$ является вполне определенным мероморфным ростком. Заменив, если надо, точку $а$ близкой точкой, можно считать, что росток $f_{a}$ регулярен. Если кривая $\gamma(t)$ не пересекает множество $A=\bigcup A_{i}$ при $t>0$, то росток $f_{a}$ мероморфно продолжается вдоль этой кривой. Пусть $B$ - проекция на сферу Римана множества полюсов функции $f$, порожденной ростком $f_{a}$. В качестве запрешенного множества ростка достаточно взять множество $A \cup B$.

5) Пусть $f_{i a}$ - ростки $\mathcal{S}$-функций, $A_{i}$-множества их особых точек и $f_{a}$ - регулярный росток, удовлетворяющий равенству

$$
f_{a}^{n}+f_{1 a} f_{a}^{n-1}+\cdots+f_{n a}=0 .
$$

Если кривая $\gamma(t)$ не пересекает множество $A=\bigcup A_{i}$ при $t>0$, то сушествует продолжение ростка $f_{a}$ вдоль этой кривой, содержащее, вообще говоря, мероморфные и алгебраические элементы. Пусть $B$ - проекция множества полюсов функции $f$ и точек разветвления ее римановой поверхности на сферу Римана $S^{2}$. В качестве запрещенного множества для ростка $f_{a}$ достаточно взять множество $A \cup B$.

6) Если коэффициенты уравнения

$$
f_{a}^{(n)}+f_{1 a} f_{a}^{(n-1)}+\cdots+f_{n a}=0
$$

регулярно продолжаются вдоль некоторой кривой $\gamma$, лежащей в конечной части сфферы Римана, то любое решение $f_{a}$ этого уравнения также регулярно продолжается вдоль кривой $\gamma$. Поэтому в качестве запрещенного множества ростка $f_{a}$ достаточно взять множество $A=\bigcup A_{i} \cup\{\infty\}$, где $A_{i}$ - множества особых точек ростков $f_{a_{i}}$. 
ЗАмЕчАниЕ. Арифметические операции и потенцирование являются примерами мероморфных операций, поэтому класс $\mathcal{S}$-функиий замкнут относительно арифметических операчий и потенцирования.

СлЕДСТВИЕ 5.9. Если многозначную функиию $f$ можно получить из однозначных $\mathcal{S}$-функиий с помощью интегрирования, дифферениирования, мероморфных операций, суперпозиций, решения алгебраических уравнений и линейньх дифференциальных уравнений, то функция $f$ имеет не более чем счетное число особых точек. В частности, функцию, имеющую несчетное число особых точек, нельзя представить в обобщенных квадратурах.

5.3. Группа монодромии. В этом пункте обсуждаются различные понятия, связанные с группой монодромии.

5.3.1. Групша монодромии с запрешенным множеством. Группа монодромии $\mathcal{S}$ функции $f$ с запрещенным множеством $A$ - это група всех перестановок листов функции $f$, которые происходят при обходе точек множества $A$. Дадим теперь точное определение.

Пусть $F_{a}$-множество всех ростков $\mathcal{S}$-функции $f$ в точке $a$, не лежашей в некотором запрешенном множестве $A$. Возьмем замкнутую кривую $\gamma$ в $S^{2} \backslash A$ с началом в точке $a$. Продолжение каждого ростка из множества $F_{a}$ вдоль кривой $\gamma$ приводит к ростку из множества $F_{a}$.

Итак, каждой кривой $\gamma$ соответствует отображение множества $F_{a}$ в себя, причем гомотопным в $S^{2} \backslash A$ кривым отвечают одинаковые отображения. Произведению кривых отвечает произведение отображений. Возникает гомоморфизм $\tau$ фундаментальной группы множества $S^{2} \backslash A$ в группу $S\left(F_{a}\right)$ взаимно однозначных преобразований множества $F_{a}$. Этот гомоморфизм будем назьвать гомоморфизмом $A$-монодромии. Группой монодромии $\mathcal{S}$-функции $f$ с запрешенным множеством $A$ или, короче, груnпой $A$-монодроми называется образ фундаментальной групшы $\pi_{1}\left(S^{2} \backslash A, a\right)$ в групе $S\left(F_{a}\right)$ при гомоморфизме $\tau$.

УТВЕРЖДЕНИЕ 5.10. 1) Группа $A$-монодромии $\mathcal{S}$-функиии не зависит от выбора точки а.

2) Группа $A$-монодромии $\mathcal{S}$-функции $f$ транзитивно действует на листах функиии $f$.

Оба утверждения просто доказываются при помощи леммы 5.6. Остановимся, например, на доказательстве второго из них.

ДокАЗАТЕЛЬСтво. Пусть $f_{1 a}$ и $f_{2 a}$ - некоторые ростки функции $f$ в точке $a$. Так как ростки $f_{1 a}$ и $f_{2 a}$ эквивалентны, то существует кривая $\gamma$, при продолжении вдоль которой ростка $f_{1 a}$ получается росток $f_{2 a}$. Согласно лемме 5.6, существует сколь угодно близкая кривая $\widehat{\gamma}$, не пересекаюшая множества $A$. Если кривая $\widehat{\gamma}$ достаточно близка к кривой $\gamma$, то соответствуюшая ей перестановка листов переводит росток $f_{1 a}$ в росток $f_{2 a}$.

5.3.2. Замкнутая групша монодромии. Зависимость групшы $A$-монодромии от выбора множества $A$ (см. п. 5.1.3) заставляет ввести тихоновскую топологию в групе перестановок листов. Оказьвается, что замыкание группы $A$-монодромии уже не зависит от множества $A$. 
Группу $S(M)$ взаимно однозначных преобразований множества $M$ мы будем рассматривать вместе со следующей топологией. Для каждого конечного множества $L \subset M$ определим окрестность $U_{L}$ тождественного преобразования как совокупность преобразований $p$ таких, что $p(l)=l$ при $l \in L$. Базис окрестностей тождественного преобразования определяется как множество окрестностей вида $U_{L}$, где $L$ пробегает все конечные подмножества $M$.

ЛЕмма 5.11 (о замыканиигрупшы монодромии). Замыкание группы монодромии $\mathcal{S}$-функии $f$ с запрещенным множеством $A$ в группе $S(F)$ всех перестановок листов функции $f$ не зависит от выбора запрещенного множества $A$.

ДокаЗАТЕЛЬСТвО. Пусть $A_{1}$ и $A_{2}$ - два запрешенных множества функции $f$ и $F_{a}-$ совокупность листов функции $f$ в точке $a, a \notin A_{1} \cup A_{2}$. Пусть $\Gamma_{1}, \Gamma_{2} \subseteq S\left(F_{a}\right)-$ группы монодромии функции $f$ с этими запрешенными множествами. Достаточно показать, что для каждой перестановки $\mu_{1} \in \Gamma_{1}$ и для каждого конечного множества $L \subseteq F_{a}$ сушествует перестановка $\mu_{2} \in \Gamma_{2}$ такая, что $\left.\mu_{1}\right|_{L}=\left.\mu_{2}\right|_{L}$. Пусть кривая $\gamma \in \pi_{1}\left(S^{2} \backslash A_{1}, a\right)$ задает перестановку $\mu_{1}$. Так как множество $L$ конечно, всякая кривая $\widehat{\gamma} \in \pi_{1}\left(S^{2} \backslash A_{1}, a\right)$, достаточно близкая к кривой $\gamma$, задает перестановку $\widehat{\mu}_{1}$, совпадаюшую с перестановкой $\mu_{1}$ на множестве $L,\left.\mu_{1}\right|_{L}=\left.\widehat{\mu}_{1}\right|_{L}$. По лемме 5.6 такую кривую $\widehat{\gamma}$ можно выбрать так, чтобы она не пересекала множества $A_{2}$. Перестановка $\widehat{\mu}_{1}$ в этом случае будет лежать в группе $\Gamma_{2}$.

Лемма делает корректньм следующее определение: замкнутой группой монодромии $\mathcal{S}$-функции $f$ называется замыкание в групе $S(F)$ группы монодромии функции с некоторым запрешенным множеством $A$.

5.3.3. Транзитивное действие грушшы на множестве и монодромная пара $\mathcal{S}$-функции. Групша монодромии функции $f$ - это не просто абстрактная группа, но транзитивная групша перестановок листов этой функции. В этом пункте напоминается алгебраическое описание транзитивных действий групшы на множествах.

Действием групш $\Gamma$ на множестве $M$ называется гомоморфизм $\tau$ группы $\Gamma$ в группу $S(M)$. Два действия $\tau_{1}: \Gamma \rightarrow S\left(M_{1}\right)$ и $\tau_{2}: \Gamma \rightarrow S\left(M_{2}\right)$ назьваются эквивалентными, если существует такое взаимно однозначное отображение $q: M_{1} \rightarrow M_{2}$, что $\bar{q} \circ \tau_{1}=\tau_{2}$, где $\bar{q}: S\left(M_{1}\right) \rightarrow S\left(M_{2}\right)$ - изоморфизм, индуцированньй отображением $q$.

Стационарной подгруппой $\Gamma_{a}$ точки $a \in M$ при действии $\tau$ назьвается подгруппа, состояшая из всех элементов $\mu \in \Gamma$ таких, что $\tau \mu(a)=a$. Действие $\tau$ назьвается транзитивныц, если для любых точек $a, b \in M$ существует $\mu \in \Gamma$ такое, что $\tau \mu(a)=$ b. Очевидно следующее утверждение.

УТВЕРЖДЕНИЕ 5.12. 1) Действие $\tau$ группь Г транзитивно, если и только если стачионарные подгруппы любых двух точек $a, b \in M$ сопряжены. Образ группь Г при транзитивном действии $\tau$ изоморфен фактор-группе $\Gamma / \bigcap_{\mu \in \Gamma} \mu \Gamma_{a} \mu^{-1}$.

2) Существует и при этом единственное с точностью до әквивалентности транзитивное действие группь $Г$ с фиксированной стационарной подгруппой некоторой точки.

Итак, транзитивные действия группы Г описьваются парами групп. Пару групп $\left[\Gamma, \Gamma_{a}\right]$, где $\Gamma_{a}-$ стационарная подгруппа некоторой точки $a$ при транзитивном действии $\tau$ групшы $\Gamma$, будем назьвать монодромной парой точки а относительно дей- 
ствия $\tau$. Групу $\tau(\Gamma) \sim \Gamma / \bigcap_{\mu \in \Gamma} \mu \Gamma_{a} \mu^{-1}$ будем называть групой монодромии пары $\left[\Gamma, \Gamma_{a}\right]$.

Гомоморфизм $A$-монодромии $\tau$ задает транзитивное действие фундаментальной групш $\pi_{1}\left(S^{2} \backslash A\right)$ в множестве $F_{a}$ листов функции $f$ в точке $a$.

Монодромную пару ростка $f_{a}$ относительно действия $\tau$ будем называть монодромной парой ростка $f_{a}$ с запрещенныцм множеством $A$. Монодромную пару ростка $f_{a}$ при действии замкнутой группой монодромии будем назьвать замкнутой монодромной парой ростка $f_{a}$. У разных ростков $\mathcal{S}$-функции $f$ монодромные пары с запрешенным множеством $A$ изоморфны, поэтому имеет смысл говорить о монодромной паре с запрешенным множеством $A$ и замкнутой монодромной паре $\mathcal{S}$-функции $f$. Замкнутую монодромную пару $\mathcal{S}$-функции $f$ будем обозначать $[f]$.

5.3.4. Почти нормальные функщии. Пару груп $\left[\Gamma, \Gamma_{0}\right], \Gamma_{0} \subseteq \Gamma$, будем называть почти нормальной парой, если сушествует конечное множество $P \subset \Gamma$ такое, что

$$
\bigcap_{\mu \in \Gamma} \mu \Gamma_{0} \mu^{-1}=\bigcap_{\mu \in P} \mu \Gamma_{0} \mu^{-1}
$$

ЛЕмма 5.13 (о дискретном действии). Образ $\tau(\Gamma)$ группы $Г$ при транзитивном действии $\tau: \Gamma \rightarrow S(M)$ является дискретной подгруппой в $S(M)$, если и только если монодромная пара $\left[\Gamma, \Gamma_{0}\right]$ некоторого әлемента $x_{0} \in M$ почти нормальна.

ДокАЗАтельство. Пусть группа $\tau(\Gamma)$ дискретна. Обозначим через $\bar{P}$ такое конечное подмножество множества $M$, что окрестность тождественного преобразования $U_{\bar{P}}$ не содержит преобразований группы $\tau(\Gamma)$, отличных от тождественного. Это означает, что пересечению $\bigcap_{x \in \bar{P}} \Gamma_{x}$ стационарных подгрупा точек $x \in \bar{P}$ отвечает тривиальное действие на множестве $M$, т.е. $\bigcap_{x \in \bar{P}} \Gamma_{x} \subseteq \bigcap_{\mu \in \Gamma} \mu \Gamma_{0} \mu^{-1}$. Группы $\Gamma_{x}$ сопряжены группе $\Gamma_{0}$, поэтому можно выбрать такое конечное множество $P \subset \Gamma$, что $\bigcap_{\mu \in P} \mu \Gamma_{0} \mu^{-1}=\bigcap_{\mu \in \Gamma} \mu \Gamma_{0} \mu^{-1}$. Обратное утверждение доказьвается аналогично.

$\mathcal{S}$-функцию $f$ будем называть почти нормальной, если ее группа монодромии дискретна. Из леммывытекает, что функция $f$ почти нормальна, если и только если почти нормальна ее замкнутая монодромная пара $[f]$.

Дифференциальной рациональной функщией от нескольких функций называется рациональная функция от этих функщий и их производных.

Лемма 5.14 (о конечно-порожденных функциях). Пусть каждый росток $\mathcal{S}$-функuии $f$ в точке а является дифференциальной рачиональной функцией от конечного числа фиксированных ростков функии $f$ в точке а. Тогда функиия $f$ почти нормальна.

Действительно, если при продолжении вдоль замкнутой кривой не изменяются фиксированные ростки функции, то не меняются и дифференциальные рациональные функции от них.

Из леммы о конечно-порожденных функциях вытекает, что любое решение линейного дифференциального уравнения с рациональными коэффициентами является почти нормальной функцией. То же самое верно и для многих других функций, естественно встречаюшихся в дифференциальной алгебре. 
5.3.5. Классы пар групп. В следующем пункте мы опишем, как изменяются замкнутые монодромные пары функций при суперпозициях, интегрированиях, дифференцированиях и т. д. Для этого нам понадобятся некоторые понятия, связанные с парами групп.

Парой групn мы всегда будем назьвать пару, состояшую из группы и некоторой ее подгрупшы. Будем отождествлять группу с парой групп, состоящей из этой групш и ее единичной подгруппы.

ОПРЕДЕЛЕНИЕ. Совокупность $\mathscr{L}$ пар будем называть почти полнылм классом пар әрупn, если

1) для каждой пары групп $\left[\Gamma, \Gamma_{0}\right] \in \mathscr{L}, \Gamma_{0} \subseteq \Gamma$, и любого гомоморфизма $\tau: \Gamma \rightarrow$ $G$, где $G$ - некоторая группа, пара груп $\left[\tau \Gamma, \tau \Gamma_{0}\right]$ также содержится в $\mathscr{L}$,

2 ) для каждой пары групп $\left[\Gamma, \Gamma_{0}\right] \in \mathscr{L}, \Gamma_{0} \subseteq \Gamma$, и любого гомоморфизма $\tau: G \rightarrow$ $\Gamma$, где $G$ - некоторая группа, пара груп $\left[\tau^{-1} \Gamma, \tau^{-1} \Gamma_{0}\right]$ также содержится в $\mathscr{L}$,

$3)$ для каждой пары групп $\left[\Gamma, \Gamma_{0}\right] \in \mathscr{L}, \Gamma_{0} \subseteq \Gamma$, и групшы $G$, наделенной $T_{2}$-топологией и содержашей групу $\Gamma, \Gamma \subseteq G$, пара груп $\left[\bar{\Gamma}, \bar{\Gamma}_{0}\right]$ также содержится в $\mathscr{L}$, где $\bar{\Gamma}, \bar{\Gamma}_{0}-$ замыкания групп $\Gamma, \Gamma_{0}$ в групше $G$.

ОПРЕДЕЛЕНИЕ. Почти полный класс пар груп будем назьвать nолнылм классом nap әрynn $\mathscr{M}$, если

1) для каждой пары груп $\left[\Gamma, \Gamma_{0}\right] \in \mathscr{M}$ и групшы $\Gamma_{1}, \Gamma_{0} \subseteq \Gamma_{1} \subseteq \Gamma$, пара групп $\left[\Gamma, \Gamma_{1}\right]$ также содержится в $\mathscr{M}$,

2) для каждых двух пар групп $\left[\Gamma, \Gamma_{1}\right],\left[\Gamma_{1}, \Gamma_{2}\right] \in \mathscr{M}$ пара групп $\left[\Gamma, \Gamma_{2}\right]$ также содержится в $\mathscr{M}$.

Минимальные почти полный и полньй классы пар груп, содержашие фиксированное множество пар групп $\mathscr{B}$, будем обозначать соответственно $\mathscr{L}\langle\mathscr{B}\rangle$ и $\mathscr{M}\langle\mathscr{B}\rangle$.

Лемма 5.15. 1) Если группа монодромии пары [Г, $\left.\Gamma_{0}\right]$ содержится в некотором полном классе пар $\mathscr{M}$, то пара $\left[\Gamma, \Gamma_{0}\right]$ также содержится в $\mathscr{M}$.

2) Если почти нормальная пара $\left[\Gamma, \Gamma_{0}\right]$ содержится в некотором полном классе пар $\mathscr{M}$, то ее группа монодромии также содержится в $\mathscr{M}$.

Остановимся на доказательстве второго утверждения. Пусть $\Gamma_{i}, i=1, \ldots, n,-$ конечное число подгруп, сопряженных с $\Gamma_{0}$, таких, что $\bigcap_{i=1}^{n} \Gamma_{i}=\bigcap_{\mu \in \Gamma} \mu \Gamma_{0} \mu^{-1}$. Пары $\left[\Gamma, \Gamma_{i}\right]$ изоморфны паре $\left[\Gamma, \Gamma_{0}\right]$, поэтому $\left[\Gamma, \Gamma_{i}\right] \in \mathscr{M}$. Пусть $\tau: \Gamma_{2} \rightarrow \Gamma-$ гомоморфизм вложения, тогда $\tau^{-1}\left(\Gamma_{1}\right)=\Gamma_{2} \cap \Gamma_{1}$, поэтому $\left[\Gamma_{2}, \Gamma_{2} \cap \Gamma_{1}\right] \in \mathscr{M}$. Класс $\mathscr{M}$ содержит пары $\left[\Gamma, \Gamma_{2}\right]$ и $\left[\Gamma_{2}, \Gamma_{2} \cap \Gamma_{1}\right]$, следовательно, $\left[\Gamma, \Gamma_{1} \cap \Gamma_{2}\right] \in \mathscr{M}$. Продолжая это рассуждение, получим, что класс $\mathscr{M}$ содержит пару $\left[\Gamma, \bigcap_{i=1}^{n} \Gamma_{i}\right]$ и вместе с ней групп $\Gamma / \bigcap_{\mu \in \Gamma} \mu \Gamma_{0} \mu^{-1}$.

УТВЕРЖДЕНИЕ 5.16 (о классе $\mathscr{L}\langle[f]\rangle$ ). Почти полный класс пар $\mathscr{L}$ содержит замкнутую монодромную пару $[f] \mathcal{S}$-функиии $f$, если и только если этот класс содержит монодромную пару функиии $f$ с запрещенным множеством $A$.

ДокАЗАТЕльство. Пусть $\left[\Gamma, \Gamma_{0}\right]$ - монодромная пара функции $f$ с запрещенным множеством $A$. Тогда $[f]=\left[\bar{\Gamma}, \bar{\Gamma}_{0}\right]$. Поэтому всякий почти полньй класс $\mathscr{L}$, содержаший пару $\left[\Gamma, \Gamma_{0}\right]$, содержит и пару $[f]$. Обратно, если $\left[\bar{\Gamma}, \bar{\Gamma}_{0}\right]$ содержится в классе $\mathscr{L}$, то и $\left[\Gamma, \Gamma_{0}\right] \in \mathscr{L}$. Действительно, топология в группе перестановок устроена таким 
образом, что $\Gamma_{0}=\Gamma \cap \bar{\Gamma}_{0}$. Поэтому пара $\left[\Gamma, \Gamma_{0}\right]$ является прообразом пары $\left[\bar{\Gamma}, \bar{\Gamma}_{0}\right]$ при вложении групшы $\Gamma$ в ее замыкание.

5.4. Основная теорема. Здесь формулируется и доказывается основная теорема топологического варианта теории Галуа.

Основная теорема 5.17. Класс $\mathcal{S}$-функиий $\widehat{\mathscr{M}}$, состоящий из $\mathcal{S}$-функиий, замкнутая монодромная пара которых лежит в некотором полном классе пар $\mathscr{M}$, замкнут относительно дифференцирования, суперпозиций и мероморфных опеpaщ,uй.

Если, кроме того, класс $\mathscr{M}$ содержит группу $\mathbb{C}$ комплексных чисел по сложению, то класс $\widehat{\mathscr{M}}$ замкнут относительно интегрирования. Если же класс $\mathscr{M}$ содержит группу $S(k)$ перестановок $k$ әлементов, то класс $\widehat{\mathscr{M}}$ замкнут относительно решения алгебраических уравнений степени не выше $k$.

Доказательство основной теоремы состоит из следующих лемм.

ЛЕмма 5.18 (о производной). Для всякой $\mathcal{S}$-функции $f$ справедливо включение $\left[f^{\prime}\right] \in \mathscr{M}\langle[f]\rangle$.

ДоказАтельство. Пусть $A$ - множество особых точек $\mathcal{S}$-функции $f$ и $f_{a}-$ росток функции $f$ в неособой точке $a$. Обозначим через $\Gamma$ фундаментальную групу $\pi_{1}\left(S^{2} \backslash A, a\right)$, через $\Gamma_{1}$ и $\Gamma_{2}$ - стационарные группы ростков $f_{a}$ и $f_{a}^{\prime}$. Группа $\Gamma_{1}$ содержится в груше $\Gamma_{2}$. Действительно, при продолжении вдоль кривой $\gamma \in \Gamma_{1}$ не изменяется росток $f_{a}$, а значит, не меняется и его производная. Из определения полного класса пар следует, что $\left[\Gamma, \Gamma_{2}\right] \in \mathscr{M}\left\langle\left[\Gamma, \Gamma_{1}\right]\right\rangle$. Воспользовавшись утверждением 5.16, получим, что $\left[f^{\prime}\right] \in \mathscr{M}\langle[f]\rangle$.

ЛЕмма 5.19 (о суперпозиции). Для всяких $\mathcal{S}$-функций $f$ и g справедливо включение $[g \circ f] \in \mathscr{M}\langle[f],[g]\rangle$.

ДокАЗАТЕЛЬСтвО. Пусть $A$ и $B$ - множества особых точек функций $f$ и $g$. Пусть $f^{-1}(B)$ - прообраз множества $B$ при многозначном соответствии, порожденном многозначной функцией $f$. Положим $Q=A \cup f^{-1}(B)$. Пусть $f_{a}$ - некоторый росток функции $f$ в точке $a \notin Q$ и $g_{b}$-некоторьй росток функции $g$ в точке $b=f(a)$. Множество $Q$ будет запрешенным для ростка $g_{b} \circ f_{a}$. Обозначим через $\Gamma$ фундаментальную групу $\pi_{1}\left(S^{2} \backslash Q, a\right)$, через $\Gamma_{1}$ и $\Gamma_{2}$ - стационарные подгрупшы ростков $f_{a}$ и $g_{b} \circ f_{a}$. Обозначим через $G$ фундаментальную группу $\pi_{1}\left(S^{2} \backslash B, b\right)$ и через $G_{0}$ - стационарную групу ростка $g_{b}$.

Определим гомоморфизм $\tau: \Gamma_{1} \rightarrow G$. Каждой кривой $\gamma \in \Gamma_{1}$ поставим в соответствие кривую $\tau \circ \gamma(t)=f(\gamma(t))$, где $f_{\gamma(t)}$ - росток, полученньй при продолжении ростка $f_{a}$ вдоль кривой $\gamma$ до точки $t$. Кривые $\tau$ о $\gamma$ будут замкнуты, так как при продолжении вдоль кривых из $\Gamma_{1}$ росток $f_{a}$ не изменяется. При гомотопии кривой $\gamma$ в множестве $S^{2} \backslash Q$ будет происходить гомотопия кривой $\tau$ ○ $\gamma$ в множестве $S^{2} \backslash B$, так как $f^{-1}(B) \subseteq Q$. Следовательно, гомоморфизм определен корректно.

Росток $g_{b} \circ f_{a}$ не будет меняться при продолжении вдоль кривых из групы $\tau^{-1}\left(G_{0}\right)$, или, другими словами, $\tau^{-1}\left(G_{0}\right) \subseteq \Gamma_{2}$. Откуда и вытекает лемма. Действительно, мы получаем включения $\Gamma \supseteq \Gamma_{2} \supseteq \tau^{-1}\left(G_{0}\right) \subseteq \tau^{-1}(G)=\Gamma_{1} \subseteq \Gamma$, из которых следует, что $\left[\Gamma, \Gamma_{2}\right] \in \mathscr{M}\left\langle\left[G, G_{0}\right],\left[\Gamma, \Gamma_{1}\right]\right\rangle$. Воспользовавшись утверждением 5.16 , получим, что $[g \circ f] \in \mathscr{M}\langle[f],[g]\rangle$. 
ЛЕмма 5.20 (об интеграле). Для всякой $\mathcal{S}$-функции $f$ справедливо включение $\left[\int f(x) d x\right] \in \mathscr{M}\langle[f], \mathbb{C}\rangle$, әде $\mathbb{C}-$ әруппа комплексных иисел по сложсению.

ДоказАТЕЛЬСтво. Пусть $A$ - множество особых точек функции $f$ и $Q=A \cup\{\infty\}$. Пусть $f_{a}$ - некоторый росток функции $f$ в точке $a \notin Q$ и $g_{a}-$ росток $\int f(x) d x$ в этой точке, $g_{a}^{\prime}=f_{a}$. В качестве запрешенного множества для ростков $f_{a}$ и $g_{a}$ можно взять множество $Q$. Обозначим через $\Gamma$ фундаментальную группу $\pi_{1}\left(S^{2} \backslash Q, a\right)$, через $\Gamma_{1}$ и $\Gamma_{2}-$ стационарные подгруппы ростков $f_{a}$ и $g_{a}$.

Определим гомоморфизм $\tau: \Gamma_{1} \rightarrow \mathbb{C}$. Каждой кривой $\gamma \in \Gamma_{1}$ поставим в соответствие число $\int_{\gamma} f(\gamma(t)) d x$, где $f_{\gamma(t)}$ - росток, полученный продолжением ростка $f_{a}$ вдоль кривой $\gamma$ до точки $t$, и $x=\gamma(t)$. Стационарная подгруппа $\Gamma_{2}$ ростка $g_{a}$ совпадает с ядром гомоморфизма $\tau$, откуда следует, что $\left[\Gamma, \Gamma_{2}\right] \in \mathscr{M}\left\langle\left[\Gamma, \Gamma_{1}\right], \mathbb{C}\right\rangle$. Воспользовавшись утверждением 5.16, получим, что $\left[\int f(x) d x\right] \in \mathscr{M}\langle[f], \mathbb{C}\rangle$.

В дальнейшем будет удобно пользоваться векторными функциями. На векторные функции непосредственно переносятся определения запрешенного множества, $\mathcal{S}$-функции, групшы монодромии.

ЛЕмма 5.21 (о векторной функции). Для всякой векторной $\mathcal{S}$-функции $\mathbf{f}=$ $\left(f_{1}, \ldots, f_{n}\right)$ справедливо равенство:

$$
\mathscr{M}\langle[\mathbf{f}]\rangle=\mathscr{M}\left\langle\left[f_{1}\right], \ldots,\left[f_{n}\right]\right\rangle .
$$

ДоказАтельство. Пусть $A_{i}$ - множества особых точек функций $f_{i}$. Множеством особых точек векторной функции $\mathbf{f}$ является множество $Q=\bigcup A_{i}$. Пусть $\mathbf{f}_{a}=$ $\left(f_{1 a}, \ldots, f_{n a}\right)$ - некоторый росток векторной функщии $\mathbf{f}$ в точке $a \notin Q$. Обозначим через Г фундаментальную группу $\pi_{1}\left(S^{2} \backslash Q, a\right)$, через $\Gamma_{i}-$ стационарные группы ростков $f_{i a}$ и через $\Gamma_{0}$ - стационарную группу векторного ростка $\mathbf{f}_{a}$. Стационарная подгруппа $\Gamma_{0}$ есть в точности $\bigcap_{i=1}^{n} \Gamma_{i}$, откуда следует, что

$$
\mathscr{M}\left\langle\left[\Gamma, \Gamma_{0}\right]\right\rangle=\mathscr{M}\left\langle\left[\Gamma, \Gamma_{1}\right], \ldots,\left[\Gamma, \Gamma_{n}\right]\right\rangle .
$$

Воспользовавшись утверждением 5.16 , получим, что

$$
\mathscr{M}\langle[\mathbf{f}]\rangle=\mathscr{M}\left\langle\left[f_{1}\right], \ldots,\left[f_{n}\right]\right\rangle .
$$

ЛЕмма 5.22 (о мероморфной операции). Для всякой векторной $\mathcal{S}$-функиии $\mathbf{f}=$ $\left(f_{1}, \ldots, f_{n}\right)$ и мероморфной функиии $F\left(x_{1}, \ldots, x_{n}\right)$ такой, что функиия $F$ ○ $\mathbf{f}$ определена, справедливо включение $[F \circ \mathbf{f}] \in \mathscr{M}\langle[\mathbf{f}]\rangle$.

ДокАЗАтЕльство. Пусть $A$ - множество особых точек функции $\mathbf{f}$ и $B$ - проекция множества полюсов функции $F \circ \mathbf{f}$ на сферу Римана. В качестве запрешенного множества функций $F$ ○ $\mathbf{f}$ и $\mathbf{f}$ можно взять множество $Q=A \cup B$. Пусть $\mathbf{f}_{a}-$ некоторьй росток функции $\mathbf{f}$ в точке $a, a \notin Q$. Обозначим через $\Gamma$ фундаментальную группу $\pi_{1}\left(S^{2} \backslash Q, a\right)$, через $\Gamma_{1}$ и $\Gamma_{2}-$ стационарные групшы ростков $\mathbf{f}_{a}$ и $F \circ \mathbf{f}_{a}$. Групп $\Gamma_{2}$ содержится в групе $\Gamma_{1}$. Действительно, при продолжении вдоль кривой $\gamma \in \Gamma_{1}$ не изменяется векторная функция, а значит, не меняется мероморфная функция от нее. Из включения $\Gamma_{2} \subseteq \Gamma_{1}$ следует, что $\left[\Gamma, \Gamma_{2}\right] \in \mathscr{M}\left\langle\left[\Gamma, \Gamma_{1}\right]\right\rangle$. Воспользовавшись утверждением 5.16, получим, что

$$
[F \circ \mathbf{f}] \in \mathscr{M}\langle[\mathbf{f}]\rangle
$$


ЛЕмма 5.23 (об алгебраической функции). Для всякой векторной $\mathcal{S}$-функции $\mathbf{f}=\left(f_{1}, \ldots, f_{n}\right)$ и алгебраической функиии у от нее, определенной равенством

$$
y^{k}+f_{1} y^{k-1}+\cdots+f_{k}=0
$$

справедливо включение $[y] \in \mathscr{M}\langle[f], S(k)\rangle$, где $S(k)$ - әруппа перестановок $k$ әлементов.

ДокаЗАТЕЛЬСтво. Пусть $A$ - множество особых точек функции $\mathbf{f}$ и $B$ - проекция множества алгебраических точек ветвления функции $y$ на сферу Римана. В качестве запрешенного множества функций $y$ и $\mathbf{f}$ можно взять множество $Q=A \cup B$. Пусть $y_{a}$ и $\mathbf{f}_{a}$ - некоторые ростки функций $y$ и $\mathbf{f}$ в точке $a \notin Q$, связанные равенством

$$
y_{a}^{k}+f_{1 a} y_{a}^{k-1}+\cdots+f_{k a}=0 .
$$

Обозначим через $Г$ фундаментальную группу $\pi_{1}\left(S^{2} \backslash Q, a\right)$ и через $\Gamma_{1}$ и $\Gamma_{2}-$ стационарные подгруппы ростков $\mathbf{f}_{a}$ и $y_{a}$. При продолжении вдоль кривой $\gamma \in \Gamma_{1}$ коэффициенты уравнения (12) не меняются, следовательно, при продолжении вдоль кривой $\gamma$ корни уравнения (12) переставляются. Возникает гомоморфизм $\tau$ группы $\Gamma_{1}$ в групшу $S(k)$, $\tau: \Gamma_{1} \rightarrow S(k)$. Группа $\Gamma_{2}$ содержится в ядре гомоморфизма $\tau$, откуда следует, что $\left[\Gamma, \Gamma_{2}\right] \in \mathscr{M}\left\langle\left[\Gamma, \Gamma_{1}\right], S(k)\right\rangle$. Воспользовавшись утверждением 5.16, получим, что

$$
[y] \in \mathscr{M}\langle[f], S(k)\rangle .
$$

Доказательство основной теоремы закончено.

5.5. Групповые препятствия к представимости в квадратурах. В этом пункте вычисляются классы пар груп, встречаюшиеся в основной теореме, и формулируются необходимые условия представимости функций в квадратурах, $k$-квадратурах и обобшенных квадратурах.

5.5.1. Вычисление некоторых классов пар групш. Основная теорема делает актуальной задачу описания наименьшего класса пар групп, содержашего группу $\mathbb{C}$ комплексных чисел по сложению, а также наименьших пар классов пар групп, содержаших соответственно группу $\mathbb{C}$ и все конечные группы, а также групп $\mathbb{C}$ и группу $S(k)$. В настояшем пункте мы приводим решение этих задач.

УТВЕРЖДЕНИЕ 5.24. Наименьший полный класс пар $\mathscr{M}\left\langle\mathscr{L}_{\alpha}\right\rangle$, содержащий заданные почти полные классы пар $\mathscr{L}_{\alpha}$, coстоит из пар групп $\left[\Gamma, \Gamma_{0}\right]$, для которых существует чепочка подгрупп $\Gamma=\Gamma_{1} \supseteq \cdots \supseteq \Gamma_{m} \subseteq \Gamma_{0}$ такая, что для любого $i, 1 \leqslant i \leqslant m-1$, пара групп $\left[\Gamma_{i}, \Gamma_{i+1}\right]$ содержится в некотором почти полном классе $\mathscr{L}_{\alpha(i)}$.

Для доказательства достаточно проверить, что пары груп $\left[\Gamma, \Gamma_{0}\right]$, удовлетворяюшие условию утверждения, во-первых, содержатся в полном классе $\mathscr{M}\left\langle\mathscr{L}_{\alpha}\right\rangle$ и, во-вторых, образуют полньй класс пар. И то и другое непосредственно вьводится из определений.

Просто проверить также следуюшие утверждения. 
УТВЕРЖДЕНИЕ 5.25. Совокупность пар групп $\left[\Gamma, \Gamma_{0}\right]$ maких, что $\Gamma_{0}$ есть нормальный делитель группь $\Gamma$ и группа $\Gamma / \Gamma_{0}$ коммутативна, образует наименьший почти полный класс пар $\mathscr{L}\langle\mathscr{A}\rangle$, содержащий класс $\mathscr{A}$ всех абелевых групп.

УТВЕРЖДЕНИЕ 5.26. Совокупность пар групп $\left[\Gamma, \Gamma_{0}\right]$ таких, что $\Gamma_{0}$ есть нормальный делитель группь $\Gamma$ и группа $\Gamma / \Gamma_{0}$ конечна, образует наименьший почти полный класс пар $\mathscr{L}\langle\mathscr{K}\rangle$, содержащий класс $\mathscr{K}$ всех конечных групп.

УТВеРЖДЕНИЕ 5.27. Совокупность пар групп $\left[\Gamma, \Gamma_{0}\right]$ maкux, ито ind $\left(\Gamma, \Gamma_{0}\right) \leqslant k$, образует почти полньй класс групп.

Класс пар групп из утверждения 5.27 будем обозначать через $\mathscr{L}\langle$ ind $\leqslant k\rangle$. Утверждение 5.27 интересно для нас в связи с характеристическим свойством подгрупा группы $S(k)$ из леммы 3.13. Цепочка подгрупп $\Gamma_{i}, i=1, \ldots, m, \Gamma=\Gamma_{1} \supseteq \cdots \supseteq \Gamma_{m} \subseteq \Gamma_{0}$ называется нормальной башней пары групn $\left[\Gamma, \Gamma_{0}\right]$, если группа $\Gamma_{i+1}$ является нормальным делителем группы $\Gamma_{i}$ при каждом $i=1, \ldots, m-1$. Совокупность фактор-групा $\Gamma_{i} / \Gamma_{i+1}$ назьвается совокупностью делителей относительно нормальной башни.

Теорема 5.28 (о классах пар $\mathscr{M}\langle\mathscr{A}, \mathscr{K}\rangle, \mathscr{M}\langle\mathscr{A}, S(k)\rangle$ и $\mathscr{M}\langle\mathscr{A}\rangle)$. 1) Пара групn $\left[\Gamma, \Gamma_{0}\right]$ принадлежит наименьшему полному классу $\mathscr{M}\langle\mathscr{A}, \mathscr{K}\rangle$, содержащему все конечные и коммутативные группь, если и только если она обладает нормальной башней, каждый делитель относительно которой является или конечной, или коммутативной группой.

2) Пара групп $\left[\Gamma, \Gamma_{0}\right]$ принадлежит наименьшему полному классу $\mathscr{M}\langle\mathscr{A}, S(k)\rangle$, содержащему әруппу $S(k)$ и все коммутативные группь, если и только если она обладает нормальной башней, каждый делитель относительно которой является или подгруппой группы $S(k)$, или коммутативной группой.

3) Пара групп $\left[\Gamma, \Gamma_{0}\right]$ принадлежит наименьшему классу $\mathscr{M}\langle\mathscr{A}\rangle$, если и только если группа монодромии этой парьь разрешима.

ДокАЗАТЕЛЬСТвО. Первый пункт теоремы вытекает из описания классов $\mathscr{L}\langle\mathscr{A}\rangle$ и $\mathscr{L}\langle\mathscr{K}\rangle$ в утверждениях 5.25 и 5.26 и из утверждения 5.24 .

Для доказательства второго пункта рассмотрим наименьший полньй класс пар груп, содержаший классы $\mathscr{L}\langle\mathscr{A}\rangle$ и $\mathscr{L}\langle$ ind $\leqslant k\rangle$. Этот класс состоит из пар групп $\left[\Gamma, \Gamma_{0}\right]$, для которых существует цепочка подгрупп $\Gamma=\Gamma_{1} \supseteq \cdots \supseteq \Gamma_{m} \subseteq \Gamma_{0}$ такая, что для любого $i, 1 \leqslant i \leqslant m-1$, либо группа $\Gamma_{i} / \Gamma_{i+1}$ коммутативна, либо $\operatorname{ind}\left(\Gamma_{i}, \Gamma_{i+1}\right) \leqslant k$ (см. утверждения $5.26,5.27$ и утверждение 5.24$)$. Описанный класс пар групп содержит группу $S(k)$ (см. лемму 3.13$)$ и все коммутативные группы и является, очевидно, наименьшим полным классом пар, обладаюшим этими свойствами. Нам осталось переформулировать ответ. Цепочку подгрупп $\Gamma=\Gamma_{1} \supseteq \cdots \supseteq \Gamma_{m} \subseteq \Gamma_{0}$ последовательно преобразуем в нормальную башню пары $\left[\Gamma, \Gamma_{0}\right]$. Пусть при $j<i$ група $\Gamma_{j+1}$ является нормальным делителем групшы $\Gamma_{j}$ и ind $\left(\Gamma_{i}, \Gamma_{i+1}\right) \leqslant k$. Обозначим через $\bar{\Gamma}_{i+1}$ наибольший нормальньй делитель групшы $\Gamma_{i}$, содержашийся в $\Gamma_{i+1}$. Ясно, что фактор-группа $\Gamma_{i} / \bar{\Gamma}_{i+1}$ является подг руппой группы $S(k)$. Вместо исходной цепочки подгрупп рассмотрим цепочку $\Gamma=G_{1} \supseteq \cdots \supseteq G_{m}=\Gamma_{0}$, в которой $G_{j}=\Gamma_{j}$ при $j \leqslant i$ и $G_{j}=\Gamma_{j} \cap \bar{\Gamma}_{i+1}$ при $j>i$. Продолжая этот процесс (не более чем $m$ раз), мы перейдем от исходной цепочки подгрупп к нормальной башне и получим описание класса $\mathscr{M}\langle\mathscr{A}, S(k)\rangle$ в нужных терминах. 
Докажем пункт 3). Согласно утверждениям 5.25 и 5.26, пара груп $\left[\Gamma, \Gamma_{0}\right]$ принадлежит классу $\mathscr{M}\langle\mathscr{A}\rangle$, если и только если существует такая цепочка $\Gamma=\Gamma_{1} \supseteq$ $\ldots \supseteq \Gamma_{m} \subseteq \Gamma_{0}$, что $\Gamma_{i} / \Gamma_{i+1}-$ коммутативная группа. Рассмотрим цепочку групп $\Gamma=G^{1} \supseteq \cdots \supseteq G^{m}$, в которой группа $G^{i+1}$ при $i=1, \ldots, m-1$ есть коммутант группы $G^{i}$. Всякий автоморфизм группы Г переводит цепочку групп $G^{i}$ в себя, поэтому каждая группа $G^{i}$ является нормальным делителем группы $Г$. Индукция по $i$ показывает, что $G^{i} \subseteq \Gamma_{i}$ и, в частности, $G^{m} \subseteq \Gamma_{m} \subseteq \Gamma_{0}$. Группа $G^{m}$ является нормальным делителем группы $\Gamma$, и так как $G^{m} \subseteq \Gamma_{0}$, то $G^{m} \subseteq \bigcap_{\mu \in \Gamma} \mu \Gamma_{0} \mu^{-1}$. В силу определения цепочки $G^{i}$ группа $\Gamma / G^{m}$ разрешима. Группа $\Gamma / \bigcap_{\mu \in \Gamma} \mu \Gamma_{0} \mu^{-1}$ разрешима как фактор-групша групшы $\Gamma / G^{m}$. Обратное утверждение (пара групп с разрешимой группй монодромии лежит в классе $\mathscr{M}\langle\mathscr{A}\rangle)$ очевидно.

УТВЕРЖДЕНИЕ 5.29. Каждая не более чем континуальная коммутативная әруппа Г принадлежит классу $\mathscr{L}\langle\mathbb{C}\rangle$.

ДокАЗАТЕльство. Комплексные числа $\mathbb{C}$ образуют векторное пространство над рациональньми числами континуальной размерности. Пусть $\left\{e_{\alpha}\right\}-$ некоторый базис этого пространства. Подгруппа $\widetilde{\mathbb{C}}$ групшы $\mathbb{C}$, натянутая на числа $\left\{e_{\alpha}\right\}$, является свободной абелевой группой с континуальным числом образующих. Всякая не более чем континуальная коммутативная группа Г есть фактор-группа групшы $\widetilde{\mathbb{C}}$ и, следовательно, $\Gamma \in \mathscr{L}\langle\mathbb{C}\rangle$.

Из утверждения 5.29 и результатов вычисления классов $\mathscr{M}\langle\mathscr{A}, \mathscr{K}\rangle, \mathscr{M}\langle S(n)\rangle$ и $\mathscr{M}\langle\mathscr{A}\rangle$ следует, что пара груп $\left[\Gamma, \Gamma_{0}\right]$ с не более чем континуальной группой $\Gamma$ принадлежит классу $\mathscr{M}\langle\mathbb{C}, \mathscr{K}\rangle, \mathscr{M}\langle\mathbb{C}, S(n)\rangle$ или $\mathscr{M}\langle\mathbb{C}\rangle$, если и только если она принадлежит классу $\mathscr{M}\langle\mathscr{A}, \mathscr{K}\rangle, \mathscr{M}\langle\mathscr{A}, S(n)\rangle$ или $\mathscr{M}\langle\mathscr{A}\rangle$.

Мы вправе ограничиться этим результатом, так как группа перестановок листов функции не более чем континуальна.

ЛЕмма 5.30. Свободная некоммутативная группа $\Lambda$ не лежит в классе $\mathscr{M}\langle\mathscr{A}, \mathscr{K}\rangle$.

ДокАЗАТЕльСтво. Предположим, что $\Lambda \in \mathscr{M}\langle\mathscr{A}, \mathscr{K}\rangle$, т.е. $\Lambda$ обладает нормальной башней $\Lambda=\Gamma_{1} \supseteq \cdots \supseteq \Gamma_{m}=e$, каждый делитель относительно которой есть конечная или коммутативная группа. Каждая группа $\Gamma_{i}$ свободна как подгруппа свободной группы (см. [28]). Группа $\Gamma_{m}=e$ коммутативна. Пусть $\Gamma_{i+1}-$ первая по номеру коммутативная група. Для любых элементов $a, b \in \Gamma_{i}$ существует нетривиальное соотношение: если $\Gamma_{i} / \Gamma_{i+1}$ коммутативна, то коммутируют, например, элементы $a b a^{-1} b^{-1}$ и $a b^{2} a^{-1} b^{-2}$, если $\Gamma_{i} / \Gamma_{i+1}$ конечна, то коммутируют некоторые степени $a^{p}, b^{p}$ элементов $a, b$. Следовательно, группа $\Gamma_{i}$ имеет не более одной образующей и поэтому коммутативна. Противоречие доказьвает, что $\Lambda \notin \mathscr{M}\langle\mathscr{A}, \mathscr{K}\rangle$.

ЛЕмма 5.31. При $k>4$ симметрическая группа $S(k)$ не лежит в классе $\mathscr{M}\langle\mathbb{C}, S(k-1)\rangle$.

ДокАЗАТЕльство. При $k>4$ знакопеременная группа $A(n)$ проста и некоммутативна. Для этой группы, очевидно, не вьполняется критерий принадлежности классу $\mathscr{M}\langle\mathbb{C}, S(k-1)\rangle$. Следовательно, и симметрическая группа $S(n)$ при $k>4$ не лежит в классе $\mathscr{M}\langle\mathbb{C}, S(k-1)\rangle$. 
ЛЕмма 5.32. Единственной транзитивной группой перестановок $k$ әлементов, натянутой на транспозиции, является симметрическая группа $S(k)$.

ДокАЗАТЕльство. Пусть групп Г есть транзитивная группа перестановок множества $M$ из $n$ элементов, натянутая на транспозиции. Подмножество $M_{0} \subseteq M$ назовем полным, если всякая перестановка множества $M_{0}$ продолжается до некоторой перестановки множества $M$ из групшы Г. Полные подмножества существуют. Например, два элемента множества $M$, переставляемые базисной транспозицией, образуют полное подмножество. Возьмем наибольшее по числу элементов полное подмножество $M_{0}$. Предположим, что $M_{0} \neq M$. Так как группа Г транзитивна, то сушествует базисная транспозиция $\mu$, переставляющая некоторый элемент $a \notin M_{0}$ с некоторым элементом $b \in M_{0}$. Группа перестановок, порожденная транспозицией $\mu$ и группй $S\left(M_{0}\right)$, есть группа $S\left(M_{0} \cup\{a\}\right)$. Множество $M_{0} \cup\{a\}$ является полным и содержит множество $M_{0}$. Полученное противоречие доказьвает, что группа Г есть группа $S(M)$.

5.5.2. Необходимые условия представимости функций в квадратурах, в $k$-квадратурах и в обобщенных квадратурах. Основная теорема (см. п. 5.4) и вычисление классов пар групп доставляют топологические препятствия к представимости функций в обобщенных квадратурах, в $k$-квадратурах и в квадратурах. В этом пункте мы соберем вместе полученную информацию. Начнем с определения класса функций, представимых при помоши однозначных $\mathcal{S}$-функций и квадратур ( $k$-квадратур, обобщенных квадратур). Как и в п. 1.2, мы определим эти классы, задав списки основных функций и допустимых операций.

Функции, представимые при помощи однозначных $\mathcal{S}$-функций и квадратур.

Список основных функиий: однозначные $\mathcal{S}$-функции.

Cnисок допустимых операций: суперпозиции, мероморфные операции, дифференцирование, интегрирование.

Функции, представимые при помощи однозначных $\mathcal{S}$-функций и $k$-квадратур. Этот класс функций определяется в точности так же. Нужно лишш к списку допустимых операций добавить операцию решения алгебраических уравнений степени $\leqslant k$.

Функции, представимые при помощи однозначных $\mathcal{S}$-функций и обобщенных квадратур. Этот класс функций определяется в точности так же. Нужно лишш к списку допустимых операций добавить операцию решения алгебраических уравнений.

Из определения видно, что класс функший, представимых с помощью однозначных $\mathcal{S}$-функций и квадратур ( $k$-квадратур, обобщенных квадратур), содержит класс функций, представимых в квадратурах ( $k$-квадратурах, обобшенных квадратурах). Ясно, что только что определенные классы функций несравненно шире, чем их классические аналоги. Поэтому, скажем, утверждение о непринадлежности функции $f$ классу функций, представимых с помошью однозначных $\mathcal{S}$-функций и квадратур, значительно сильнее, чем утверждение о непредставимости $f$ в квадратурах.

УТВЕРЖДЕНИЕ 5.33. Класс функиий, представимых с помощью однозначных 
$\mathcal{S}$-функиий и квадратур ( $\mathcal{S}$-функиий.

Это утверждение немедленно вытекает из теоремы о замкнутости класса $\mathcal{S}$-функций (см. п. 5.2.2).

РЕЗУЛЬТАТ ОБ ОБОБШЕННЫХ КВАДРАТУРАХ. Замкнутая монодромная пара $[f]$ функции $f$, представимой в обобщенных квадратурах, обладает нормальной башней, каждый делитель относительно которой есть или конечная, или коммутативная группа. Более того, этому условию удовлетворяет замкнутая монодромная пара $[f]$ всякой функиии $f$, представимой при помощи однозначных $\mathcal{S}$-функиий и обобщенных квадратур. Если дополнительно известно, что функиия $f$ почти нормальна, то указанному условию удовлетворяет и группа монодромии функции $[f]$.

РЕЗУЛЬТАТ о $k$-КВАДРАТУРАХ. Замкнутая монодромная пара $[f]$ функиии $f$, представимой в k-квадратурах, обладает нормальной башней, каждый делитель относительно которой есть или подгруппа группь $S(k)$, или коммутативная группа. Более того, әтому условию удовлетворяет замкнутая монодромная пара $[f]$ всякой функции $f$, представимой при помощи однозначных $\mathcal{S}$-функиий и $k$-квадратур. Если дополнительно известно, что функция $f$ почти нормальна, то указанному условию удовлетворяет и группа монодромии функu, uu $[f]$.

РЕЗУЛЬТАТ О КВАДРАТУРАХ. Замкнутая группа монодромии функиии $f$, представимой в квадратурах, разрешима. Более того, разрешима замкнутая группа монодромии всякой функиии $f$, представимой при помощи однозначных $\mathcal{S}$-функций и квадратур.

Для доказательства этих результатов достаточно применить основную теорему к классам $\mathcal{S}$-функций $\widehat{\mathscr{M}}\langle\mathbb{C}, \mathscr{K}\rangle, \widehat{\mathscr{M}}\langle\mathbb{C}, S(k)\rangle$ и $\widehat{\mathscr{M}}\langle\mathbb{C}\rangle$ и воспользоваться вычислением классов $\mathscr{M}\langle\mathbb{C}, \mathscr{K}\rangle, \mathscr{M}\langle\mathbb{C}, S(k)\rangle$ и $\mathscr{M}\langle\mathbb{C}\rangle$.

Приведем теперь примеры функций, не представимых в обобщенных квадратурах. Пусть риманова поверхность функции $f$ является универсальной накрывающей над областью $S^{2} \backslash A$, где $S^{2}$ - сфера Римана и $A$ - конечное множество, содержашее не меньше трех точек. Тогда функиия $f$ не выражается при помощи однозначных $\mathcal{S}$-функций и обобщенных квадратур. Действительно, функция $f$ является почти нормальной функцией. Замкнутая группа монодромии функции $f$ свободна и некоммутативна, так как свободна и некоммутативна фундаментальная группа области $S^{2} \backslash A$.

Пример 1. Рассмотрим функцию $f$, конформно отображающую верхнюю полуплоскость на треугольник с нулевьми углами, ограниченньй дугами окружностей. Функция $f$ обратна к модулярной функции Пикара. Риманова поверхность функции $f$ является универсальной накрьвающей над сферой без трех точек, поэтому функция $f$ не выражается при помощи однозначных $\mathcal{S}$-функций и обобщенных квадратур.

Отметим, что функция $f$ тесно связана с эллиптическими интегралами

$$
K_{1}(k)=\int_{0}^{1} \frac{d x}{\sqrt{\left(1-x^{2}\right)\left(1-k^{2} x^{2}\right)}} \text { и } \quad K_{2}(k)=\int_{0}^{\frac{1}{k}} \frac{d x}{\sqrt{\left(1-x^{2}\right)\left(1-k^{2} x^{2}\right)}} \text {. }
$$


Каждые две из функций $K_{1}, K_{2}$ и $f$ выражаются друг через друга при помоши квадратур (см. [13]). Поэтому каждый из интегралов $K_{1}$ и $K_{2}$ не выражсется при помощи однозначных $\mathcal{S}$-функиий и обобщенных квадратур.

Пример 1 допускает существенное обобщение: в работе [21] (см. также [22]) перечислены все многоугольники, ограниченные дугами окружностей, на которые можно отобразить верхнюю полуплоскость функцией, представимой в обобщенных квадратурах.

Пример 2. Пусть $f-k$-значная алгебраическая функция с некратными точками ветвления, расположенными в разных точках сферы Римана. При $k>4$ функиия $f$ не выражается при помощи однозначных $\mathcal{S}$-функций $и(k-1)$-квадратур, суперпозичий и мероморфных операций. $B$ частности, функиия $f$ не представима в $(k-1)$-квадратурах.

Действительно, при обходе некратной точки ветвления функции $f$ происходит транспозиция в множестве листов этой функции. Группа монодромии функции $f$ является транзитивной группой перестановок, натянутой на транспозиции, т.е. группой $S(k)$. При $k>4$ группа $S(k)$ не лежит в классе $\mathscr{M}\langle\mathbb{C}, S(k-1)\rangle$.

В статьях [23]-[25] топологические результаты о непредставимости функций в квадpaтурах ( $k$-квадратурах и обобщенных квадратурах) обобщены на случай функций многих комплексных переменных.

5.5.3. Классы особых множеств и обобщение основной теоремы. В $\S 5$ рассматривались $\mathcal{S}$-функции, т.е. многозначные аналитические функции комплексного переменного, множества особых точек которых не более чем счетны. Пусть $\mathcal{S}$ - класс всех не более чем счетных подмножеств сферы Римана $S^{2}$. Перечислим свойства класса $\mathcal{S}$, которыми мы сушественно пользовались:

1) если $A \in S$, то множество $S^{2} \backslash A$ всюду плотно и локально линейно связно,

2) существует непустое множество $A$ такое, что $A \in \mathcal{S}$,

3) если $A \in \mathcal{S}$ и $B \subseteq A$, то $B \in \mathcal{S}$,

4) если $A_{i} \in \mathcal{S}, i=1,2, \ldots$, то $\bigcup_{i=1}^{\infty} A_{i} \in \mathcal{S}$,

5) пусть $U_{1}$ и $U_{2}$ - открытые подмножества сферы и $f: U_{1} \rightarrow U_{2}$ - обратимое аналитическое отображение, тогда если $A \subseteq U_{1}$ и $A \in \mathcal{S}$, то $f(A) \in \mathcal{S}$.

Полным классом множеств будем назьвать всякое множество подмножеств сферы Римана, удовлетворяюшее свойствам 1)-5). Многозначную аналитическую функцию будем назьвать $Q$-функщией, если множество ее особых точек лежит в некотором полном классе множеств $Q$. На $Q$-функции переносятся все определения и теоремы из $\S 5$. Так, например, справедлив следующий вариант основной теоремы.

ВАРИАНТ ОСНОВНОЙ ТЕОРЕМЫ. Для всякого полного класса множеств $Q u$ полного класса пар $\mathscr{M}$ класс $\widehat{M}$, состоящий из всех $Q$-функиий $f$, для которьх $[f] \in \mathscr{M}$, замкнут относительно дифференцирования, суперпозичий и мероморфныз операций.

Если дополнительно $\mathbb{C} \in \mathscr{M}$, то класс $Q$-функций $\widehat{\mathscr{M}}$ замкнут относительно интегрирования.

Если же $S(k) \in \mathscr{M}$, то класс $Q$-функций $\widehat{\mathscr{M}}$ замкнут относительно решения алгебраических уравнений степени не выше $k$. 
Приведем пример полных классов множеств. Пусть $X_{\alpha}-$ множество всех подмножеств сферы Римана, имеющих нулевую хаусдорфову меру веса $\alpha$. Несложно показать, что при $\alpha \leqslant 1$ множество $X_{\alpha}$ образует полный класс подмножеств сферы.

Отметим, что новая формулировка основной теоремы позволяет усилить все отрицательные результаты. Остановимся, например, на результате о непредставимости функций в квадратурах. (Результаты о непредставимости в $k$-квадратурах и в обобщенных квадратурах обобщаются точно так же.) Определим следующий класс функций.

Функции, представимые при помощи однозначных $X_{1}$-функций и квадратур.

Список основных функций: однозначные $X_{1}$-функции.

Список допустимых операций: суперпозиции, мероморфные операции, дифференцирование, интегрирование.

Согласно новой формулировке основной теоремы, $\mathcal{S}$-функиия, имеющая неразрешимую группу монодромии, не только непредставима в квадратурах, но непредставима и при помощи однозначных $X_{1}$-функций и квадратур.

\section{$\S$ 6. Разрешимость в квадратурах линейных дифференциальных уравнений типа Фукса и топологический вариант теории Галуа}

6.1. Теория Пикара-Вессио для уравнений типа Фукса. В этом пункте показывается, что топология расположения над комплексной плоскостью римановой поверхности общего решения линейного дифференщиального уравнения типа Фукса целиком отвечает за разрешимость уравнения в явном виде.

6.1.1. Группа монодромии линейного дифференшиального уравнения, ее связь $c$ групшой Галуа. Рассмотрим линейное дифференциальное уравнение

$$
y^{(n)}+r_{1} y^{(n-1)}+\cdots+r_{n} y=0,
$$

где $r_{i}$ - рациональные функции комплексного переменного $x$. Полюсы рациональных функций $r_{i}$ и точка $\infty$ называются особыми точками уравнения (13).

В окрестности неособой точки $x_{0}$ решения уравнения образуют $n$-мерное пространство $V^{n}$. Возьмем теперь произвольную кривую $\gamma(t)$ на комплексной плоскости, ведущую из точки $x_{0}$ в точку $x_{1}$ и не проходяшую через особые точки $a_{i}$. Решения уравнения будут аналитически продолжаться вдоль кривой, оставаясь при этом решениями уравнения. Поэтому каждой кривой $\gamma$ отвечает линейное отображение $M_{\gamma}$ пространства решений $V_{x_{0}}^{n}$ в точке $x_{0}$ в пространство решений $V_{x_{1}}^{n}$ в точке $x_{1}$.

Если пошевелить кривую $\gamma$, не задевая при этом особых точек и оставляя закрепленными концы, то отображение $M_{\gamma}$ меняться не будет. Замкнутым кривым будет отвечать линейное преобразование пространства $V^{n}$ в себя. Совокупность всех таких линейных преобразований пространства $V^{n}$ образует группу, которая и назьвается группой монодромии уравнения (13). Итак, группа монодромии уравнения это группа линейных преобразований решений, которые возникают при обходе особых точек. Группа монодромии уравнения характеризует многозначность его решений. 
Лемма 6.1. 1) Группа монодромии почти каждого решения уравнения (13) изоморфна группе монодромии этого уравнения.

2) Монодромная пара каждого решения уравнения (13) почти нормальна.

ДокАЗАТЕльство. Второе утверждение леммы вытекает из леммы 5.14. Остановимся на доказательстве первого утверждения. Группа монодромии уравнения (13) это матричная групп, содержащая не более чем счетное число элементов. Для каждого нетождественного элемента этой группы множество его неподвижных точек является собственным подпространством конечномерного пространства решений уравнения (13). Множество решений, остаюшихся неподвижными, хотя бы для одного нетождественного преобразования из групшы монодромии имеет нулевую меру в пространстве решений (так как объединение не более чем счетного числа собственных подпространств конечномерного пространства имеет в этом пространстве нулевую меру). Группа монодромии всех остальных решений уравнения (13) изоморфна групе монодромии уравнения.

В окрестности неособой точки $x_{0}$ существуют $n$ линейно независимых решений $y_{1}, \ldots, y_{n}$ уравнения (13). В этой окрестности можно рассмотреть поле функций $\mathscr{R}\left\langle y_{1}, \ldots, y_{n}\right\rangle$, полученное присоединением к полю рациональных функций $\mathscr{R}$ всех решений $y_{i}$ и всех их производных.

Каждое преобразование $M_{\gamma}$ пространства решений из групшы монодромии можно продолжить до автоморфизма всего поля $\mathscr{R}\left\langle y_{1}, \ldots, y_{n}\right\rangle$. Действительно, вместе с функциями $y_{1}, \ldots, y_{n}$ вдоль кривой $\gamma$ будет мероморфно продолжаться каждьй элемент поля $\mathscr{R}\left\langle y_{1}, \ldots, y_{n}\right\rangle$. Это продолжение и дает требуемьй автоморфизм, так как при продолжении сохраняются арифметические операции и дифференщирование, а рациональные функции возврашаются к своему прежнему значению из-за однозначности.

Итак, группа монодромии уравнения (13) лежст в группе Галуа этого уравнения над полем рачиональных функиий.

Поле инвариантов группы монодромии - это подполе поля $\mathscr{R}\left\langle y_{1}, \ldots, y_{n}\right\rangle$, состояшее из однозначных функций. В отличие от алгебраических уравнений, для дифференциальных уравнений поле инвариантов относительно действия групы монодромии может быть больше, чем поле рациональных функций.

Например, для дифференциального уравнения (13), у которого все коэффициенты $r_{i}(x)$ являются полиномами, все решения - целые функции. Но, конечно, решения таких уравнений далеко не всегда полиномиальны. Дело здесь в том, что решения дифференциальных уравнений могут расти при подходе к особым точкам экспоненщиальньм образом. Известен широкий класс линейных дифференщиальных уравнений, для которых такого осложнения нет, т.е. для которых решения при подходе к каждой особой точке (вдоль любого сектора с вершиной в этой точке) растут не быстрее чем степенным образом. Дифференциальные уравнения, обладающие этим свойством, называются дифференщиальными уравнениями типа Фукса (см. [11], [16]). Для дифференциальных уравнений типа Фукса справедлива следующая теорема Фробениуса.

Теорема 6.2 (Фробениуса). Подполе дифференциального поля $\mathscr{R}\left\langle y_{1}, \ldots, y_{n}\right\rangle$, состоящее из однозначных функиий, для дифференциальных уравнений типа Фукса совпадает с полем рациональньх функций. 
Прежде чем доказьвать теорему Фробениуса, остановимся на ее непосредственных следствиях.

СлЕДСТВИЕ 6.3. Алгебраическое замыкание группы монодромии $M$ (т.е. наименьшая алгебраическая группа, содержсащая $M$ ) уравнения типа Фукса совпадает с әруппой Галуа этого уравнения над полем рациональных функций.

ДокАЗАТЕЛЬСтво. Следствие вытекает из теоремы Фробениуса и основной теоремы дифференциальной теории Галуа (см. п. 4.3).

ТеОРема 6.4. Линейное дифференциальное уравнение типа Фукса решается в квадратурах, в $k$-квадратурах или в обобщенньх квадратурах, если и только если его группа монодромии соответственно разрешима, $k$-разрешима или почти разрешима.

Доказательство вытекает из теоремы Пикара-Вессио (см. п. 4.5) и предыдушего следствия.

Дифференциальная теория Галуа доказывает тем самым два результата.

1) Если группа монодромии дифференииального уравнения типа Фукса разрешима (k-разрешима, почти разрешима), то әто уравнение решается в квадратурах ( в к-квадратурах, в обобщенных квадратурах).

2) Если группа монодромии дифференциального уравнения типа Фукса неразрешима (не k-разрешима, не почти разрешима), то это уравнение не решается в квадратурах (в $k$-квадратурах и в обобщенных квадратурах).

Первьй из этих результатов не требует основной теоремы теории Галуа и, по существу, относится к линейной алгебре. Дело в том, что группу автоморфизмов дифференциального поля $R\left\langle y_{1}, \ldots, y_{n}\right\rangle$, оставляюших на месте только поле рациональных функций, не нужно специально конструировать. Такой группой является группа монодромии. Поэтому для доказательства разрешимости в квадратурах и в обобщенных квадратурах уравнений типа Фукса с разрешимой или с почти разрешимой группой монодромии достаточно воспользоваться линейно-алгебраическими рассуждениями из п. 4.7. Для доказательства разрешимости в $k$-квадратурах уравнение типа Фукса с $k$-разрешимой группой монодромии этих линейно-алгебраических рассуждений недостаточно. Нужно еше воспользоваться теорией Галуа алгебраических расширений поля рациональных функций (см. утверждение 4.21). Впрочем, теория Галуа алгебраических расширений поля $R$ весьма наглядна и геометрична (см. п. 5.1.1).

Наша теорема позволяет усилить отрицательный результат 2). Об этом - в п. 6.2.4. А сейчас перейдем к доказательству теоремы Фробениуса.

6.1.2. Доказательство теоремы Фробениуса. Мы покажем, что каждая однозначная функция из дифференциального поля $\mathscr{R}\left\langle y_{1}, \ldots, y_{n}\right\rangle$ мероморфна на сфере Римана и, следовательно, рациональна. Пусть $p \in S$ - особая точка уравнения типа Фукса и $x$ - локальный параметр около этой точки такой, что $x(p)=0$. Согласно теории Фукса, около точки $p$ каждое решение $y$ представляется в виде конечной суммы $y=\sum f_{\alpha k} x^{\alpha} \ln ^{k} x$, где $f_{\alpha k}-$ мероморфные функции около точки $p$. Ясно, что функции, представимые в виде $\sum f_{\alpha k} x^{\alpha} \ln ^{k} x$, где функции $f_{\alpha k}$ мероморфны около точки $p$, образуют дифференциальное кольцо, содержашее поле функций, мероморфных около точки $p$. Нам нужно показать, что частное двух функций из этого дифференциального кольца является однозначной функцией около точки $p$, если и только если эта 
функция мероморфна. Доказательство этого факта основано на формулируемом ниже утверждении 6.5. Нам понадобятся следующие обозначения: $U(0, \varepsilon)-\varepsilon$-окрестность точки 0 на комплексной плоскости; $\widehat{U}(0, \varepsilon)$ - проколотая $\varepsilon$-окрестность точки 0 , $\widehat{U}(0, \varepsilon)=U(0, \varepsilon) \backslash\{0\} ; M(0, \varepsilon)$ и $\widehat{M}(0, \varepsilon)$ - поля мероморфных функций в областях $U(0, \varepsilon)$ и $\widehat{U}(0, \varepsilon)$.

Два мероморфных ростка $f_{a}$ и $g_{b}$ называются әквивалентными над областью $U$, $a, b \in U$, если росток $g_{b}$ получается из ростка $f_{a}$ при продолжении вдоль некоторой кривой, лежащей в области $U$.

Определим теперь кольцо $K_{a}(0, \varepsilon)$. Мероморфный росток $f_{a}$, заданный в точке $a \in$ $\widehat{U}(0, \varepsilon)$, принадлежит кольцу $K_{a}(0, \varepsilon)$, если:

1) росток $f_{a}$ мероморфно продолжается вдоль всех кривых, лежаших в $\widehat{U}(0, \varepsilon)$,

2) комплексное векторное пространство, натянутое на все мероморфные ростки в точке $a$, эквивалентные над окрестностью $\widehat{U}(0, \varepsilon)$ ростку $f_{a}$, конечномерно.

Кольцо $K_{a}(0, \varepsilon)$ содержит поле $\widehat{M}(0, \varepsilon)$ и является векторным пространством над ним.

УТВЕРЖДЕНИЕ 6.5 (о базисе). При любом выборе ветвей функций $\ln x$ u $x^{\alpha}$, $[\operatorname{Re} \alpha]=0$, ростки $x_{a}^{\alpha} \ln _{a}^{k} x, k=0,1,2, \ldots$, образуют базис пространства $K_{a}(0, \varepsilon)$ над полем $\widehat{M}(0, \varepsilon)$.

Сначала докажем лемму.

ЛЕмма 6.6. Ростки $1, \ln _{a} x, \ldots, \ln _{a}^{k} x, \ldots$ линейно независимь над полем $\widehat{M}(0, \varepsilon)$.

ДокАЗАТЕльСТво. Действительно, существование нетривиального соотношения $\sum a_{k} \ln _{a}^{k} x=0, a_{k} \in \widehat{M}(0, \varepsilon)$, влечет за собой конечнозначность функции $\ln x$ в окрестности нуля.

Доказательство утверждения основывается на рассмотрении оператора монодромии $A: K_{a}(0, \varepsilon) \rightarrow K_{a}(0, \varepsilon)$, которьй сопоставляет каждому ростку его продолжение вдоль замкнутой кривой, обходящей точку 0.

Лемма 6.7. Ростки $x_{a}^{\alpha} \ln _{a}^{k} x,[\operatorname{Re} \alpha]=0, k=0,1, \ldots, n-1$, образуют базис $\boldsymbol{\theta}$ пространстве $\operatorname{ker}(A-\lambda E)^{n}$, где $\lambda$ и а связаны соотношением $\lambda=e^{2 \pi i \alpha}$.

ДокАЗАТЕЛЬСтво. Заметим, что пространство $\operatorname{ker}(A-\lambda E)$ не более чем одномерно. Действительно, если $A f_{a}=\lambda f_{a}$ и $A g_{a}=\lambda g_{a}$, то $A\left(f_{a} / g_{a}\right)=f_{a} / g_{a}$. Следовательно, росток $\psi_{a}=f_{a} / g_{a}$ является ростком некоторой функции $\psi$ из поля $\widehat{M}(0, \varepsilon)$ и $f_{a}=\psi g_{a}$. Поэтому пространство $\operatorname{ker}(A-\lambda E)^{n}$ имеет размерность не вьше $n$. $\mathrm{C}$ другой стороны, легко проверить, что в этом пространстве лежат ростки $x_{a}^{\alpha} \ln _{a}^{k} x$, $[\operatorname{Re} \alpha]=0, k=0,1, \ldots, n-1$. Согласно лемме 6.6 , эти ростки линейно независимы и поэтому образуют базис пространства $\operatorname{ker}(A-\lambda E)^{n}$.

Пространства $\operatorname{ker}(A-\lambda E)^{n}$ при разных $\lambda$ имеют нулевое пересечение. Поэтому все ростки $x_{a}^{\alpha} \ln _{a}^{k} x$ линейно независимы. Покажем, что всякий росток $f_{a}$ из пространства $K_{a}(0, \varepsilon)$ можно разложить по этим функциям. По определению росток $f_{a}$ лежит в некотором конечномерном пространстве $V$, инвариантном относительно оператора монодромии. Пусть $\widetilde{A}$ - ограничение оператора $A$ на пространстве $V$. Согласно 
линейной алгебре, пространство $V$ раскладьвается в прямую сумму подпространств $\operatorname{ker}(\widetilde{A}-\lambda E)^{n_{\lambda}}$, где $\lambda$ - собственное значение оператора $\widetilde{A}$ и $n_{\lambda}-$ его кратность. Из леммы 6.7 вытекает, что всякий элемент пространства $V$ раскладывается по векторам $x_{a}^{\alpha} \ln _{a}^{k} x$.

ЗАмЕчАНИЕ. Выбор разных ветвей функций $\ln x$ и $x^{\alpha}$ приводит к разным базисам пространства $K_{a}(0, \varepsilon)$. Коэффициенты разложения векторов из таких базисов по другому базису являются комплексными числами.

ОПРЕДЕЛЕНИЕ. 1) Мероморфный росток $f_{a}, a \in \widehat{U}(0, \varepsilon)$, имеет над окрестностью $\widehat{U}(0, \varepsilon)$ иелую фуксову особенность, если $f_{a} \in K_{a}(0, \varepsilon)$ и коэффищиенты разложения ростка $f_{a}$ по базису $x_{a}^{\alpha} \ln _{a}^{k} x$ мероморфны, т.е. если

$$
f_{a}=\sum f_{\alpha, k} \ln _{a}^{k} x \cdot x_{a}^{\alpha}, \quad \text { где } f_{\alpha, k} \in M(0, \varepsilon) .
$$

2) Мероморфный росток $f_{a}, a \in \widehat{U}(0, \varepsilon)$, имеет над окрестностью $\widehat{U}(0, \varepsilon)$ фуксову особенность, если он представим в виде частного двух ростков $\psi_{a}, g_{a}$, имеюших над $\widehat{U}(0, \varepsilon)$ целую фуксову особенность, $f_{a}=\psi_{a} / g_{a}$.

С ЛЕДСТВИЕ 6.8. Росток $f_{a} \in K_{a}(0, \varepsilon)$ имеет фуксову особенность над окрестностью $\widehat{U}(0, \varepsilon)$, если и только если он имеет челую фуксову особенность над әтой окрестностью.

ДокаЗАТельство. Росток $f_{a} \in K_{a}(0, \varepsilon)$, следовательно, $f_{a}=\sum r_{\alpha, k} x_{a}^{\alpha} \ln _{a}^{k} x$, где $r_{\alpha, k} \in \widehat{M}(0, \varepsilon)$ - коэффициенты разложения ростка $f_{a}$ по базису. Росток $f_{a}$ имеет также фуксову особенность, поэтому справедливо равенство

$$
\frac{\sum p_{\alpha, k} x_{a}^{\alpha} \ln _{a}^{k} x}{\sum q_{\alpha, k} x_{a}^{\alpha} \ln _{a}^{k} x}-\sum r_{\alpha, k} x_{a}^{\alpha} \ln _{a}^{k} x=0,
$$

где $p_{\alpha, k}, q_{\alpha, k}$ - некоторые элементы поля $M(0, \varepsilon)$. Умножим последнее равенство на $\sum q_{\alpha, k} x_{a}^{\alpha} \ln _{a}^{k} x$, раскроем скобки и приведем, если надо, ростки $x_{a}^{\beta} \ln _{a}^{k} x$ к виду $x^{n} \cdot x_{a}^{\alpha} \ln _{a}^{k} x$, где $n$ - целое и $[\operatorname{Re} \alpha]=0$. Так как ростки $x_{a}^{\alpha} \ln _{a}^{k} x$ линейно независимы над полем $\widehat{M}(0, \varepsilon)$, то равенство эквивалентно системе уравнений, полученной приравниванием к нулю коэффициентов при этих функщиях. Полученная система представляет собой систему линейных уравнений относительно функций $r_{\alpha, k}$ с коэффициентами из поля $M(0, \varepsilon)$. Система имеет единственное решение, так как функции $r_{\alpha, k}$ определены однозначно. Следовательно, функции $r_{\alpha, k}$ лежат в поле $M(0, \varepsilon)$.

СЛЕДСТВИЕ 6.9. Если росток $f_{a}$ мероморфной в окрестности $\widehat{U}(0, \varepsilon)$ функции $f$ имеет над этой окрестностью фуксову особенность, то функиия $f$ мероморфна в окрестности $\widehat{U}(0, \varepsilon)$.

ДокАЗАТЕльство. Росток $f_{a} \in K_{a}(0, \varepsilon)$ и его разложение по базису имеет вид $f_{a}=f \cdot 1$. Согласно следствию 6.8 , росток $f_{a}$ имеет целую фуксову особенность и поэтому $f \in M(0, \varepsilon)$.

Следствие 6.9 завершает доказательство теоремы Фробениуса. 
6.1.3. Группа монодромии систем линейных дифференщиальных уравнений, ее связь с группой Галуа. Результаты из п. 6.1.1 автоматически переносятся на системы линейных дифференциальных уравнений с регулярными особыми точками.

Рассмотрим линейное дифференщиальное уравнение

$$
\mathbf{y}^{\prime}=\mathbf{A}(x) \mathbf{y}
$$

где $\mathbf{y}=\left(y_{1}(x), \ldots, y_{n}(x)\right), \mathbf{A}(x)=\left(a_{i, j}(x), 1 \leqslant i, j \leqslant n\right)$ - матрица рациональных функций и $x$ - комплексная переменная. Пусть $a_{1}, \ldots, a_{k}$ - полюсы матрицы $\mathbf{A}(x)$. В окрестности неособой точки $x_{0}, x_{0} \neq \infty, x_{0} \neq a_{i}, i=1, \ldots, k$, решения уравнения (14) образуют $n$-мерное пространство $V^{n}$. Возьмем теперь произвольную кривую $\gamma(t)$ на комплексной плоскости, ведушую из точки $x_{0}$ в точку $x_{1}$ и не проходяшую через особые точки $a_{i}, \gamma(0)=x_{0}, \gamma(1)=x_{1}, \gamma(t) \neq a_{i}$. Решения уравнения будут аналитически продолжаться вдоль кривой, оставаясь при этом решениями уравнения. Поэтому каждой кривой $\gamma$ отвечает линейное отображение $M_{\gamma}$ пространства решений $V_{x_{0}}^{n}$ в точке $x_{0}$ в пространство решений $V_{x_{1}}$ в точке $x_{1}$.

Если пошевелить кривую $\gamma$, не задевая при этом особых точек и оставляя закрепленными концы, то отображение $M_{\gamma}$ меняться не будет. Замкнутым кривым будет отвечать линейное преобразование пространства $V^{n}$ в себя. Совокупность всех таких линейных преобразований пространства $V^{n}$ образует группу, которая и назьвается группой монодромии уравнения (14). Итак, группа монодромии уравнения это группа линейных преобразований решений, которые возникают при обходе особых точек. Группа монодромии уравнения характеризует многозначность его решений.

Лемма 6.10. 1) Группа монодромии почти каждого решения системь (14) совпадает с группой монодромии системы (14). 2) Монодромная пара каждой компоненты каждого решения системы (14) почти нормальна. 3) Если группа монодромии системы (14) не лежит в некотором полном классе пар групп $\mathscr{M}$, то монодромная пара одной из компонент почти каждого решения этой системы не лежит в $\mathscr{M}$.

ДокАзАтельство. Первые два утверждения леммы доказываются так же, как лемма 6.1. Утверждение 3) вытекает из 1) и леммы 5.21.

В окрестности неособой точки $x_{0}$ сушествуют все решения $\mathbf{y}_{1}, \ldots, \mathbf{y}_{n}$ уравнения (14). В этой окрестности можно рассмотреть дифференщиальное поле функций $R\left\langle\mathbf{y}_{1}, \ldots, \mathbf{y}_{n}\right\rangle$, полученное присоединением к полю рациональных функций $R$ всех компонент $y_{i 1}, \ldots, y_{i n}$ всех решений у ${ }_{i}$ и всех их производных $y_{i j}^{(p)}$.

Каждое преобразование $M_{\gamma}$ пространства решений из группы монодромии можно продолжить до автоморфизма всего дифференциального поля $\mathscr{R}\left\langle\mathbf{y}_{1}, \ldots, \mathbf{y}_{n}\right\rangle$ над полем $R$. Действительно, вместе с вектор-функциями $\mathbf{y}_{1}, \ldots, \mathbf{y}_{n}$ вдоль кривой $\gamma$ будет мероморфно продолжаться каждьй элемент поля $\mathscr{R}\left\langle\mathbf{y}_{1}, \ldots, \mathbf{y}_{n}\right\rangle$. Это продолжение и дает требуемьй автоморфизм, так как при продолжении сохраняются арифметические операции и дифференцирование, а рациональные функции возврашаются к своему прежнему значению из-за однозначности.

Особая точка уравнения (14) называется регулярной, если в любом секторе с вершиной в особой точке все решения при подходе к этой точке растут не быстрее чем степенным образом (см. [16], [11]). Известно, что около регулярной особой точки каждая 
компонента каждого решения имеет целую фуксову особенность (см. определение из п. 6.1.2). Уравнение (14) назьвается регулярным, если все его особые точки (включая $\infty)$ регулярны. Для регулярного уравнения (14) все однозначные функции из поля $\mathscr{R}\left\langle\mathbf{y}_{1}, \ldots, \mathbf{y}_{n}\right\rangle$ являются рациональными функциями.

ТеОРема 6.11. Для регулярной системы линейных дифференциальных уравнений (14) дифференциальное поле $\mathscr{R}\left\langle\mathbf{y}_{1}, \ldots, \mathbf{y}_{n}\right\rangle$ является расширением Пикара-Вессио поля $R$. Группа Галуа этого расширения является алгебраическим замыканием группы монодромии системы уравнений (14).

ДокАЗАТЕльство. На дифференциальном поле $\mathscr{R}\left\langle\mathbf{y}_{1}, \ldots, \mathbf{y}_{n}\right\rangle$ группа монодромии действует как группа изоморфизмов с полем инвариантов $R$. Поле $\mathscr{R}\left\langle\mathbf{y}_{1}, \ldots, \mathbf{y}_{n}\right\rangle$ порождено над $R$ конечномерньм $\mathbb{C}$-линейным пространством, инвариантным относительно действия монодромии, а именно линейным пространством, натянутьм на все компоненты всех решений уравнения (14). Теперь теорема вытекает из следствия 4.5.

ТЕОРема 6.12. Каждая компонента каждого решения регулярной системь линейных дифференциальных уравнений выражсается в квадратурах, в $k$-квадратурах и в обобщенных квадратурах, если и только если группа монодромии системы соответственно разрешима, k-разрешима или почти разрешима.

Доказательство вытекает из теоремы 4.12 (Пикара-Вессио) и из предыдущей теоремы. Как и в случае уравнения типа Фукса, “положительная" часть теоремы, относяшаяся к разрешимости системы, доказывается, в основном, при помоши линейной алгебры (см. п. 4.7). А отрицательную часть теоремы значительно усиливает топологический вариант теории Галуа (см. п. 6.2.4).

6.2. Теория Галуа систем линейных дифференциальных уравнений типа Фукса с малыми коэффициентами. Оказьвается, для систем уравнений типа Фукса с достаточно малыми коэффициентами условия разрешимости становятся абсолютно явными [15].

6.2.1. Системы уравнений типа Фукса. Среди систем регулярных линейных дифференциальных уравнений выделяются системы линейных дифференциальных уравнений типа Фукса. Это уравнение вида $\mathbf{y}^{\prime}=\mathbf{A}(x) \mathbf{y}$, где матрица $\mathbf{A}(x)$ не имеет кратных полюсов и обрашается в нуль на бесконечности. Другими словами, это уравнения вида

$$
\mathbf{y}^{\prime}=\sum_{p=1}^{k} \frac{A_{p}}{x-a_{p}} \mathbf{y},
$$

где $A_{p}$ - комплексная $(n \times n)$-матрица, а $\mathbf{y}=\left(y_{1}, \ldots, y_{n}\right)$-вектор в $\mathbb{C}^{n}$. Точки $a_{p}$ называются полюсами, а матрицы $A_{p}$ - матрищами-вычетами системы уравнений типа Фукса.

Для систем уравнений типа Фукса, как и для других регулярных систем дифференциальных уравнений, алгебраическое замыкание группы монодромии совпадает с әруппой Галуа порожденного системой уравнений расширения Пикара-Вессио поля рациональных функиий (см. п. 6.1.3).

Лаппо-Данилевский развил теорию аналитических функций от матрищ и применил ее к дифференциальньм уравнениям [29]. Нам понадобятся результаты Лаппо-Данилевского относительно систем уравнений типа Фукса, которые мы будем использовать 
в виде следствия, приведенного в конце этого пункта.

Возьмем неособую точку $x_{0} \neq a_{p}$. Зафиксируем $k$ кривых $\gamma_{1}, \ldots, \gamma_{k}$ так, чтобы кривая $\gamma_{p}$ начиналась в точке $x_{0}$, подходила к полюсу $a_{p}$, обходила его и возврашалась назадв точку $x_{0}$. Кривым $\gamma_{1}, \ldots, \gamma_{k}$ отвечают матрищы монодромии $M_{1}, \ldots, M_{k}$. Очевидно, что матрицы $M_{1}, \ldots, M_{k}$ порождают группу монодромии. При фиксации кривых матрицы монодромии зависят лишш от матриц-вычетов. Эта зависимость изучалась Лаппо-Данилевским.

Во-первых, он показал, что матрицы монодромии $M_{p}$ - целые функции матрищ-вычетов $A_{j}$. Точнее, существуют специальные ряды с комплексными коэффициентами

$$
M_{p}=E+2 \pi i A_{p}+\sum_{1 \leqslant i, j \leqslant k} c_{i, j} A_{i} A_{j}+\cdots
$$

от матрии $A_{1}, \ldots, A_{k}$, выражсающие матрищь монодромии $M_{p}$ и сходящиеся при любых матрицах $A_{1}, \ldots, A_{k}$.

Хотя матрица монодромии $M_{p}$ зависит от всех матриц-вычетов $A_{j}$, ее собственные числа определяются только по собственным числам матрицы-вычета $A_{p}$.

ТеОрема 6.13 [16], [11]. Пусть $\left\{\mu_{m}\right\}$ - набор собственных чисел матрицы $A_{p}$. Тогда $\left\{e^{2 \pi i \mu_{m}}\right\}-$ набор собственных чисел матрицы $M_{p}$.

Знаменитая проблема Римана-Гильберта - это вопрос о разрешимости обратной задачи, т.е. вопрос о существовании уравнений типа Фукса с заданным набором матриц монодромии. Для почти всякого набора матриц монодромии задача Римана-Гильберта разрешима. Традиционно считалось, что этот классический результат переносится на любые наборы матрищ монодромии. Однако, как обнаружил А. А. Болибрух [10], [11], это не так. Он предъявил пример набора матриц монодромии, для которых проблема Римана-Гильберта неразрешима.

Лаппо-Данилевский показал, что в предположении малости матрищ-вычетов $A_{j}$ матришы-вычеты $A_{j}$ - однозначные аналитические функшии матрищ монодромии $M_{p}$. А именно, он показал, что если ограничиться уравнениями типа Фукса с достаточно малыми матрицами-вычетами $\left\|A_{j}\right\|<\varepsilon, \varepsilon=\varepsilon\left(n, a_{1}, \ldots, a_{k}\right)$, то для достаточно близких к $E$ матриц монодромии $M_{p},\left\|M_{p}-E\right\|<\varepsilon$, задача Римана-Гильберта имеет единственное решение. Более того, существуют специальные ряды с комплексными коэффициентами

$$
A_{p}=-\frac{1}{2 \pi i} E+\frac{1}{2 \pi i} M_{p}+\sum_{1 \leqslant i, j \leqslant k} b_{i j} M_{i} M_{j}+\cdots
$$

от матрии, $M_{1}, \ldots, M_{k}$, выражсающие матрищь-вьчеть $A_{p}$ и сходящиеся при $\left\|M_{p}-E\right\|<\varepsilon$.

Ряды (16) получаются обрашением рядов (15). Этот результат является своеобразной теоремой о неявной функции (для аналитических отображений с некоммутативными переменными).

Теорию Лаппо-Данилевского мы будем использовать в форме следующего утверждения. 
СлЕДСТВИЕ 6.14. Матрицы монодромии лежат в алгебре с единицей, натянутой на матрицы-вычеты. Обратно, если матрицы-вычеты достаточно мальи и матрицы монодромии достаточно близки $\kappa$ E, то матрицы-вычеты лежат в алгебре с единицей, натянутой на матрицы монодромии.

6.2.2. Групш, порожденные матрицами, близкими к единичной. В этом пункте доказывается аналог теоремы Ли для матричных групп, порожденных матрицами, близкими к единичньм. Напомним формулировку теоремы Жордана.

ТеОРема 6.15 (Жордан). Конечная группа $G$ линейных преобразований $n$-мерного пространства обладает диагональным нормальным делителем $G_{d}$ ограниченного индекса, $\operatorname{ind}\left(G, G_{d}\right) \leqslant J(n)$.

Известны различные явные оценки сверху чисел $J(n)$. (Например, Шур показал, что $\left.J(n) \leqslant(\sqrt{8 n}+1)^{2 n^{2}}-\sqrt{8 n}-1\right)^{2 n^{2}}$, см. [37].)

УТВЕРЖДЕНИЕ 6.16. Существует иелое число T(n) такое, что подгруппа $G$ в $G L(n)$ обладает разрешимым нормальным делителем конечного индекса, если и только если она обладает треугольным нормальным делителем индекса $\leqslant T(n)$.

ДокАЗАТЕльство. Теорема Ли гарантирует существование у грушы $G$ треугольного нормального делителя $G_{l}$ конечного индекса. Действительно, достаточно положить $G_{l}=G \cap \bar{G}_{0}$, где $\bar{G}_{0}$ - компонента связности единищы алгебраического замькания $\bar{G}$ групшы $G$. Однако индекс $G_{l}$ может быть как угодно велик. Так, например, для групшы $\mathbb{Z}_{k}$ корней $k$-й степени из единицы этот индекс равен $k$ при $n=1$. Мы будем увеличивать нормальный делитель $G_{l}$, оставляя его треугольным. Отметим, что нам достаточно доказать существование треугольной подгрупш ограниченного индекса, так как подгруппа индекса $k$ содержит нормальньй делитель индекса $\leqslant k$ ! Доказательство будем вести индукцией по размерности $n$. Если группа $G$ обладает инвариантным пространством $V^{k}$ размерности $k, 0<k<n$, мы сможем сделать индукционньй шаг. Действительно, група $G$ в этом случае действует и на пространстве $V^{k}$ размерности $k$, и на фактор-пространстве $V^{n} / V^{k}$ размерности $(n-k)$. По индукции можно считать, что группа $G$ обладает нормальным делителем индекса $\leqslant T(k) T(n-k)$, которьй треуголен как в $V^{k}$, так и в $V^{n} / V^{k}$, т.е. треуголен в $V^{n}$.

Нормальный делитель $G_{l}$ приводится к треугольному виду и поэтому обладает ненулевьм максимальным собственным подпространством $V^{k}$. Возникают два случая: $V^{k} \subset V^{n}$ и $V^{k}=V^{n}$. Рассмотрим первьй случай: $V^{k} \subset V^{n}$. Обозначим через $\widetilde{G}_{l}$ подгруппу $G$, состояшую из всех преобразований, для которых $V^{k}$ инвариантно (в этом месте и происходит увеличение нормального делителя $G_{l}$ ). Докажем, что $\operatorname{ind}\left(G, \widetilde{G}_{l}\right) \leqslant n$. Действительно, групша перестановок $G$ переставляет максимальные собственные пространства всякого своего нормального делителя и, в частности, $G_{l}$. Однако максимальных собственных пространств не может быть больше $n$. Отсюда и вытекает нужное соотношение $\operatorname{ind}\left(G, \widetilde{G}_{l}\right) \leqslant n$. Для окончания доказательства достаточно применить к группе $\widetilde{G}_{l}$ индукционный шаг. Рассмотрим второй случай: $V^{k}=V^{n}$, т.е. $G_{l}$ состоит из матрищ $\lambda E$. Можно считать, что груша $G$ состоит из матриц с единичным детерминантом. Действительно, в противном случае можно рассмотреть группу, составленную из матриц $(\operatorname{det} A)^{-1} A$. Нормальньй делитель $D_{l}$ при этом предположении конечен (поскольку $\lambda^{n}=1$ ). Группа $G$ тоже конечна, так как 
$\operatorname{ind}\left(G, G_{l}\right)<\infty$. Для окончания доказательства достаточно воспользоваться теоремой Жордана.

УТВЕРЖДЕНИЕ 6.16'. Существует челое число $D(n)$ maкое, что подгруппа $G$ в $G L(n)$ обладает диагональным нормальнымм делителем конечного индекса, если и только если она обладает диагональнымм нормальным делителем индекса $\leqslant D(n)$.

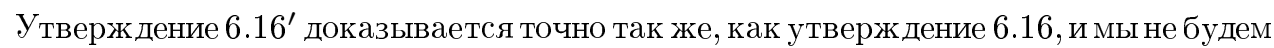
останавливаться на его доказательстве. Числа $T(n)$ и $D(n)$ также допускают явную оценку сверху (ср. [37]).

Лемма 6.17. Уравнение $X^{N}=A,\|A-E\|<\varepsilon,\|X-E\|<\varepsilon$, в котором $X$ и $A$ - комплексные $(n \times n)$-матрицы, близкие $\kappa E$, имеет единственное решение, если $\varepsilon=\varepsilon(n, N)$ достаточно мало. При этом каждое инвариантное пространство $V$ матрицы $A$ будет инвариантно и для матрицы $X$.

ДОКАЗАТЕЛЬСТВО. Положим $B=A-E$ и

$$
X=E+\frac{1}{N} B+\frac{1}{2} \frac{1}{N}\left(\frac{1}{N}-1\right) B^{2}+\cdots .
$$

При $\|B\|<1$ ряд сходится и $X^{N}=A$. Выберем теперь $\varepsilon=\varepsilon(n, N)$ столь малым, чтобы теорема о неявной функции гарантировала бы единственность решения. Пространство $V$ будет инвариантно относительно $B=A-E$ и, следовательно, относительно $X$.

Лемма 6.18. Пусть $N$-е степени всех матрич из группь $G$ лежсат в некоторой алгебраической группе $L$, тогда группа $G \cap L$ имеет в группе $G$ конечный индекс.

ДокАЗАтельство. Рассмотрим алгебраическое замькание $\bar{G}$ групшы $G$. Легко видеть, что если $X \in \bar{G}$, то $X^{N} \in L$. Обозначим через $\bar{G}_{0}$ и $L_{0}$ компоненты связности единицыгрупп $\bar{G}$ и $L$. Если $A$ лежит в группе $L_{0}, A=e^{M}$, то уравнение $X^{N}=A$ имеет решение в этой же группе. Действительно, достаточно положить $X=e^{\frac{M}{N}}$. Но уравнение $X^{N}=A$ имеет единственное решение при матрицах $A$ и $X$, близких к $E$. Отсюда следует, что $\bar{G}_{0} \subseteq L_{0} \subseteq L$. Лемма теперь вытекает из того, что ind $\left(\bar{G}, \bar{G}_{0}\right)<\infty$.

ЗАмечАниЕ. При $L=e$ лемма 6.18 преврашается в теорему Бернсайда: матричная группа с тождеством $X^{N}=$ е конечна.

УТВЕРЖДЕНИЕ 6.19. Существует челое число $N(n)$ maкое, что подгруппа $G$ в $G L(n)$ обладает разрешимым нормальным делителем конечного индекса, если и только если все матрицы $A^{N(n)}, A \in G$, одновременно приводятся к треугольному виду.

ДокАЗАТЕЛЬСтво. В одну сторону утверждение 6.19 вытекает из утверждения 6.16, если положить $N(n)=T(n)$ !. Для доказательства в другую сторону нужно применить лемму 6.18 для групшы $G$ и групшы треугольных матриц $L$.

Аналогично доказывается следующее утверждение. 
УТВЕРЖДЕНИЕ 6.19'. Существует иелое число $N(n)$ такое, что подгруппа $G$ в $G L(n)$ обладает диагональным нормальным делителем конечного индекса, если и только если все матрицы $A^{N(n)}, A \in G$, одновременно приводятся $к$ диагональному виду.

ТеОрема 6.20. Существует положительное число $\varepsilon(n)>0$, такое, что подгруппа $G$ в $G L(n)$, порожсденная матрицами $A_{\alpha}$, близкими к единичной, $\left\|E-A_{\alpha}\right\|<\varepsilon(n)$, обладает разрешимым нормальным делителем конечного индекса, если и только если все матрицы $A_{\alpha}$ одновременно приводятся $\kappa$ треугольному виду.

ДокАЗАТЕЛЬСтво. Выберем $\varepsilon(n)>0$ столь малым, что для уравнения

$$
X^{N(n)}=A,
$$

$\|E-X\|<\varepsilon(n)$, выполнены условия леммы 6.17. По утверждению 6.19 все матрищы $A_{\alpha}^{N(n)}$ должны приводиться к треугольному виду. Но по лемме 6.17 инвариантные пространства матриц $A_{\alpha}^{N(n)}$ и $A_{\alpha}$ совпадают. Поэтому матрицы $A_{\alpha}$ тоже приводятся к треугольному виду.

Аналогично доказывается следуюшее утверждение.

УТВЕРЖДЕНИЕ 6.21. Существует положительное число $\varepsilon(n)>0$ такое, что подгруппа $G$ в $G L(n)$, порожденная матрицами $A_{\alpha}$, близкими $к$ единичной, $\left\|E-A_{\alpha}\right\|<\varepsilon(n)$, обладает диагональным нормальным делителем конечного индекса, если и только если все матрицы $A_{\alpha}$ одновременно приводятся $\kappa$ диагональному виду.

ЗАмЕЧАНИЕ. В теореме 6.20 и в утверждении 6.21 можно ослабить требование близости матрищ $A_{\alpha}$ к единичной. Достаточно ограничиться близостью в топологии Зарисского. Скажем, что матрища $A k$-резонансна, если у нее найдутся разные собственные числа $\lambda_{1}$ и $\lambda_{2}$, связанные соотношением $\lambda_{1}=\varepsilon_{k} \lambda_{2}, \varepsilon_{k}^{k}=1, \varepsilon_{k} \neq 1$. Все $k$-резонансные матрицы образуют алгебраическое множество, не содержашее единицы. Достаточно требовать, чтобы матрицы $A_{\alpha}$ не были $N(n)$-резонансньми.

6.2.3. Явные критерии разрешимости. Перейдем к явному критерию разрешимости. Начнем с двух простых лемм.

Лемма 6.22. Система типа Фукса п-го порядка

$$
\dot{\mathbf{y}}=\sum_{i=1}^{k} \frac{A_{i}}{x-a_{i}} \mathbf{y}
$$

с достаточно мальми коэффициентами $\left\|A_{i}\right\|<\varepsilon=\varepsilon\left(n, a_{1}, \ldots, a_{k}\right)$ решается в обобщ,нных квадратурах, если и только если ее матрицы монодромии $M_{i}$ треугольны.

ДоКАЗАТЕЛЬСТВО. Группа монодромии системы порождена матрицами монодромии $M_{i}$. Если матрищы-вычеты $A_{i}$ малы, $\left\|A_{i}\right\|<\varepsilon$, то матрищы $M_{i}$ будут близки к $E$. Выберем $\varepsilon=\varepsilon\left(n, a_{1}, \ldots, a_{k}\right)$ столь мальм, чтобы для матрищ монодромии $M_{1}, \ldots, M_{k}$ вьполнялись условия теоремы 6.20. В силу этой теоремы у группы монодромии сушествует разрешимьй нормальный делитель конечного индекса, если и только если матрицы $M_{1}, \ldots, M_{k}$ треугольны. Теперь осталось воспользоваться теоремой 6.12 . 
Лемма 6.23. Для системы типа Фукса треугольность и диагональность группы Галуа әквивалентны тому жсе условию на матрицы монодромии $M_{1}, \ldots, M_{k}$.

ДокАЗАТЕЛЬСтво. Группа монодромии порождена матрицами монодромии $M_{1}, \ldots, M_{k}$ и треугольна или диагональна вместе с ними. Теперь лемма вытекает из того, что для уравнения типа Фукса группа Галуа совпадает с алгебраическим замьканием групшы монодромии (см. п. 6.1.3).

КРИТЕРИЙ РАЗРЕШИМОСТИ. По набору полюсов $a_{1}, \ldots, a_{k}$ и порядку $n$ можно указать число $\varepsilon\left(n, a_{1}, \ldots, a_{k}\right)$ такое, что условия разрешимости для систем n-го порядка типа Фукса

$$
\dot{\mathbf{y}}=\sum_{i=1}^{k} \frac{A_{i}}{x-a_{i}} \mathbf{y}
$$

с мальми коэффициентами, $\left\|A_{i}\right\|<\varepsilon\left(n, a_{1}, \ldots, a_{k}\right)$, принимают явный вид.

Именно, система решается:

1) в квадратурах или в обобщенных квадратурах ${ }^{3}$, если и только если матрицы $A_{i}$ (в некотором базисе) треугольны;

2) в интегралах и алгебраических функииях или в интегралах и радикалах ${ }^{3}$, если и только если матрицы $A_{i}$ треугольны и их собственные числа раииональнь;

3) в интегралах, если и только если матицы $A_{i}$ треугольны и их собственные числа равны нулю;

4) в әкспонентах интегралов и в алгебраических функииях или в әкспонентах интегралов ${ }^{3}$, если и только если матрицы $A_{i}$ диагональны;

5) в алгебраических функииях или в радикалах ${ }^{3}$, если и только если матриць $A_{i}$ диагональны и их собственнье числа рациональны;

6) в рациональных функциях, если и только если все матрицы $A_{i}$ равнь нулю.

ДокАЗАТЕльство. Выберем $\varepsilon\left(n, a_{1}, \ldots, a_{k}\right)$ столь малым, чтобы выполнялись условия леммы 6.23 и чтобы матрицы-вычеты выражались через матрицы монодромии (см. п. 6.2.1).

Каждый из видов разрешимости влечет за собой разрешимость в обобщенных квадратурах. Разрешимость в обобшенных квадратурах при наших предположениях влечет треугольность матриц монодромии (лемма 6.22) и, следовательно, треугольность групшы Галуа (лемма 6.23). Поэтому мы находимся в рамках применимости критерия, приведенного в конще п. 4.8. Нам нужно превратить условия на группу Галуа из этого критерия в условия на матрицы-вычеты $A_{i}$.

Условия на групу Галуа из критерия п. 4.8 эквивалентны тем же условиям на матрицы монодромии $M_{1}, \ldots, M_{k}$. Частично мы это проверили в лемме 6.23 . Остальная проверка столь же несложна.

В предположениях нашей теоремы условие принадлежности матриц монодромии $M_{1}, \ldots, M_{k}$ некоторой алгебре с единицей, например алгебре треугольных или диагональных матрищ, эквивалентно тому же условию на матрицы-вычеты $A_{1}, \ldots, A_{k}$ (следствие 6.14).

\footnotetext{
3Эти виды разрешимости различаются, если не ограничивать величины коэфффициентов.
} 
Собственные числа матрищы $M_{i}$ будут корнями из единицы или единицами, если и только если собственные числа матрицы $A_{i}$ - рациональные или целые числа (см. п. 6.2.1).

Теперь наш критерий вытекает из критерия п. 4.8.

ЗАмечАнИЕ. На конференщии, посвященной 100-летию А.Н. Колмогорова, А.А. Болибрух сказал мне, что в условиях критерия разрешимости требование малости матриц $A_{i}$ можно ослабить. Достаточно лишь требовать, чтобь собственные числа этих матрии, были малы. Это был наш последний разговор с Андреем.

6.2.4. Сильная неразрешимость уравнений. Топологический вариант теории Галуа позволяет усилить классические результаты о неразрешимости уравнений в явном виде.

Группа монодромии алгебраической функции совпадает с групой Галуа соответствуюшего расширения Галуа поля рациональных функций (см. п. 5.1.2). Поэтому, согласно теории Галуа, 1) алгебраическая функиия представима в радикалах, если и только если ее группа монодромии разрешима; 2) алгебраическая функиия выражается через рациональнье функции при помощи радикалов и решения алгебраических уравнений степени $k$, если и только если ее группа монодромии k-разрешима.

Из наших результатов (см. п. 5.5.2) вытекает следуюшее утверждение.

СЛЕДСТВИЕ 6.24. 1) Если группа монодромии алгебраического уравнения над полем рачиональных функций неразрешима, то его решение не принадлежит классу функиий, представимых при помощи однозначных $\mathcal{S}$-функиий и квадраmyp.

2) Если группа монодромии алгебраического уравнения не $k$-разрешима, то его решение не принадлежит классу функций, представимых при помощи однозначных $\mathcal{S}$-функиий и $k$-квадратур.

Аналогичным образом усиливаются результаты о неразрешимости в явном виде из пाг. 6.1.1, 6.1.3 и 6.2.3.

СлЕДСТВИЕ 6.25. Если группа монодромии линейного дифференциального уравнения над полем рациональных функиий неразрешима (не k-разрешима, не почти разрешима), то общее решение уравнения не принадлежст классу функций, представимых при помощи однозначных $\mathcal{S}$-функций и квадратур ( $k$-квадратур, обобщенных квадратур).

СлЕДСТВИЕ 6.26. Если группа монодромии системы линейных дифференииальных уравнений над полем рачиональных функиий неразрешима (не $k$-разрешима, не почти разрешима), то по крайней мере одна из компонент почти каждого решения не лежит в классе функиий, представимых с помощью однозначных $\mathcal{S}$-функчий и квадратур ( $k$-квадратур, обобщенных квадратур).

СлЕДСТВИЕ 6.27. Если система дифференциальньх уравнений типа Фукса $c$ маленькими коэффициентами не является треугольной, то по крайней мере одна из компонент почти каждого решения не лежит в классе функиий, представимьх с помощью однозначных $\mathcal{S}$-функций и квадратур (k-квадратур, обобщенных көадратур). 


\section{СПИСОК ЛИТЕРАТУРЫ}

[1] В.Б. Алексеев. Теорема Абеля в задачах и решениях. М.: Изд-во МЦНМО, 2001.

[2] В. И. Арнольд. Алгебраическая неразрешимость проблемы устойчивости по Ляпунову и проблемы топологической классификации особых точек аналитической системы диффференциальных уравнений // Функц. анализ и его прил. 1970. Т. 4. № 3. С. 1-9.

[3] В.И. Арнольд. Суперпозиции // А.Н. Колмогоров. Избранные труды. Математика и механика. М.: Наука, 1985. С. 444-451.

[4] В.И. Арнольд. Топологическое доказательство трансцендентности абелевых интегралов в "Математических началах натуральной философии" Ньютона // Историко-математические исследования. 1989. №31. С. 7-17.

[5] V.I. Arnol'd. Problèmes résolubles et problèmes irrésolubles analytiques et géométriques // Passion des Formes. Dynamique Qualitative Sémiophysique et Intelligibilité. Dédié à R. Thom. Fontenay-St Cloud: ENS Éditions, 1994. P. 411-417.

[6] V.I. Arnol'd. Sur quelques problèmes de la théorie des systèmes dynamiques // Topol. Methods Nonlinear Anal. 1994. V. 4. № 2. Р. 209-225. Рус. пер.: В. И. Арнолшд. О некоторых задачах теории динамических систем // Владимир Игоревич Арнолд. Избранное-60. М.: Фазис, 1997. С. 533-551.

[7] В.И. Арнольд. И.Г. Петровский, топологические проблемы Гильберта и современная математика // УМН. 2002. Т. 57. № 4. С. 197-207.

[8] В.И. Арнольд, О. А. Олейник. Топология действительных алгебраических многообразий // Вестник МГУ. Сер. 1. Матем., мех. 1979. Т. 6. С. 7-17.

[9] V.I. Arnol'd, V.A. Vassill'ev. Newton's Principia read 300 years later // Notices Amer. Math. Soc. 1989. V. 36. № 9. P. 1148-1154. Addendum: ibid. 1990. V. 37. № 2. P. 144.

[10] А. А. Болибрух. Обратные задачи монодромии аналитической теории дифференциальных уравнений // Математические события XX века. М.: Фазис, 2003. С. 53-79.

[11] А. А. Болибрух. Фуксовы дифференциальные уравнения и голоморфные расслоения. М.: Изд-во МЦНМО, 2000.

[12] Д. Б. Фукс, А.Т. Фоменко, В.Л. Гутенмахер. Гомотопическая топология. М.: Изд-во Моск. ун-та, 1969.

[13] В.В.Голубев. Лекции по аналитической теории дифференциальных уравнений. М.-Л.: Гостехиздат, 1950.

[14] А. Гурвиц, Р. Курант. Теория функций. М.: Наука, 1968.

[15] Ю. С. Ильяшенко, А. Г. Хованский. Теория Галуа систем дифференциальных уравнений типа Фукса с малшми коэффициентами // Препринт ИПМ АН СССР № 117. М., 1974.

[16] Э. Л. Айнс. Обыкновенные диффференциальные уравнения. Харьков: Гос. научно-техн. изд-во, 1939.

[17] И. Капланский. Введение в дифференциальную алгебру. М.: Мир, 1959.

[18] А. Г. Хованский. О представимости алгеброидных функций суперпозициями аналитических функций и алгеброидных функций одной переменной // Функц. анализ и его прил. 1970. T. 4. № 2. С. 74-79.

[19] А. Г. Хованский. О суперпозициях голоморфных функций с радикалами // УМН. 1971. T. 26. № 2. C. 213-214.

[20] А. Г. Хованский. О представимости функций в квадратурах // УМН. 1971. Т. 26. № 4. C. $251-252$.

[21] А.Г. Хованский. О представимости функций в квадратурах // Дисс. ... канд. физ.-матем. наук. М.: МИАН, 1973.

[22] A. Khovanskij. Topological obstructions for representability of functions by quadratures // J. Dynam. Control Systems. 1995. V. 1. № 1. P. 91-123.

[23] А. Г. Хованский. О продолжаемости многозначных аналитических функций на аналитическое подмножество // Функц. анализ и его прил. 2001. Т. 35. № 1. С. 62-73.

[24] А. Г. Хованский. О монодромии многозначной функции на ее множестве ветвления // Функц. анализ и его прил. 2003. Т. 37. № 2. С. 65-74.

[25] А. Г. Хованский. Многомерные результаты о непредставимости функций в квадратурах // Функц. анализ и его прил. 2003. Т. 37. № 4. С. 74-85. 
[26] E. R. Kolchin. Algebraic matric groups and the Picard-Vessiot theory of homogeneous linear ordinary differential equations // Ann. of Math. (2). 1948. V. 49. P. 1-42.

[27] E. R. Kolchin. Galois theory of differential fields // Amer. J. Math. 1953. V. 75. P. 753-824.

[28] А. Г. Курош. Лекции по общей алгебре. М.: Физматгиз, 1962.

[29] И. А. Лаппо- Данилевский. Применение функций от матриц к теории линейных систем обыкновенных дифференциальных уравнений. М.: ГИТТЛ, 1957.

[30] J. Liouville. Sur la détermination des intégrales dont la valeur est algébrique // J. Ecole Polytech. Paris. 1833. V. 14. P. 124-193.

[31] J. Liouville. Mémoire sur l'intégration d'une classe de fonctions transcendentes // J. Reine Angew. Math. 1835. V. 13. № 2. P. 93-118.

[32] J. Liouville. Mémoire sur l'intégration d'une classe d'équations différentielles du second ordre en quantités finies explicites // J. Math. Pures Appl. Sér. I. 1839. V. 4. P. 423-456.

[33] J. F. Ritt. Integration in Finite Terms. Liouville's Theory of Elementary Methods. New York: Columbia Univ. Press, 1948.

[34] M. Rosenlicht. Liouville's theorem on functions with elementary integrals // Pacific J. Math. 1968. V. 24. P. 153-161.

[35] M. Rosenlicht. On Liouville's theory of elementary functions // Pacific J. Math. 1976. V. 65. № 2. P. 485-492.

[36] M.F. Singer. Formal solutions of differential equations // J. Symbolic Comput. 1990. V. 10. № 1. P. 59-94.

[37] M. F. Singer. Liouvillian solutions of $n$th order homogeneous linear differential equations // Amer. J. Math. 1981. V. 103. № 4. P. 661-682.

University of Toronto, Canada,

Поступила в редакцию

Московский независимьй университет, Россия,

30.03 .2004

Институт системного анализа РАН, Россия

E-mail: askold@math.toronto.edu 